\title{
Biological Matter in Microfluidic Environment - from Single Molecules to Self-Assembly
}

\author{
Dissertation \\ zur Erlangung des Doktorgrades \\ der Mathematisch-Naturwissenschaftlichen Fakultäten \\ der Georg-August-Universität zu Göttingen
}

vorgelegt von

Sarah Friederike Köster

aus Reutlingen

Göttingen 2006 
D7

Referent:

Prof. Dr. S. Herminghaus

Koreferent:

Prof. Dr. T. Salditt

Tag der mündlichen Prüfung: 13. Juni 2006 


\section{Abstract}

The interior as well as the exterior of cells is governed by networks composed of fibrous proteins. The mesh size of these networks is on the order of micrometers and therefore distinguishes microfluidics as an excellent tool to gain insight into principal mechanisms of single molecule behavior, on the one hand, and the interplay and self-assembly of the network constituents, on the other hand. Here, we present new results derived from biomimetic investigations of two different systems, namely single molecule experiments on actin, one of the most important intracellular proteins, and in situ observation of the fibril formation of collagen I, the most abundant extracellular protein. The use of microfluidic channels fabricated by means of soft photolithography as the principle tool for our experiments enables us to manipulate the molecules via confining wall potentials and hydrodynamic flow fields, analyze their mechanical behavior, and observe time and spatially resolved reactions. Furthermore, microfluidics is very well suited for combination with different observation methods such as fluorescence microscopy, polarized light microscopy, and X-ray microdiffraction.

Analyzing single fluctuating actin filaments under the influence of confinement yields a thorough characterization of the mechanics of the system. The biomacromolecules are observed by means of fluorescence microscopy. We find that the behavior of the biopolymers depends on their contour length $L$ and the influence of the microfluidic environment. The confining energy is considered as a parabolic wall potential. Thus, we succeed to remarkably well describe the competition between bending energy and confining energy. Moreover, the results are consistent with Monte Carlo simulations and with scaling laws for the deflection length $\lambda$ and the segment distribution in the channels.

The experiments on collagen I give insight into the dynamic evolution of the hierarchical organization of native collagen fibrils. We use a hydrodynamic focusing and diffusive mixing device to establish a stable $\mathrm{pH}$-gradient within the microfluidic channels. Therefore, we are able to perform non-equilibrium measurements in the laminar flow and observe different stages of the self-assembly process at different po- 
sitions within the same system. We characterize the system on a microscopic length scale by availing the birefringent properties of collagen. Additionally, using X-ray microdiffraction the dynamic formation of critical subunits of collagen fibrils can be observed. Furthermore, we demonstrate that finite element method simulations provide a good description of our experimental results regarding diffusive phenomena, influence of the solution viscosity on the flow profile, and $\mathrm{pH}$ distribution.

The experiments presented here elucidate the principle understanding of the studied biological systems and furthermore show the ability of microfluidic tools to advance the diverse field of life science. 


\section{Inhalt}

Sowohl das Zellinnere als auch die zellumgebende Matrix sind von Netzwerken aus Faserproteinen durchzogen. Da die Maschenweite dieser Netzwerke im Bereich weniger Mikrometer liegt, ist die Mikrofluidik besonders geeignet, um einerseits das prinzipielle Verhalten von Einzelmolekülen, umgeben von einem Geflecht aus Polymeren, und andererseits das Zusammenspiel und die Organisation der Netzwerkbestandteile zu charakterisieren. In dieser Arbeit stellen wir neuartige Ergebnisse aus biomimetischen Untersuchungen zweier unterschiedlicher Systeme vor: Einzelmolekülexperimente an Aktin, einem der bedeutendsten intrazellulären Proteine, und in situ Beobachtungen der Faserbildung von Kollagen I, dem häufigsten extrazellulären Protein. Wir verwenden mikrofluidische Messzellen, die mit den Methoden der ,,weichen Lithographie“ aus elastischen Kunststoffen hergestellt werden. Dadurch sind wir in der Lage, Makromoleküle durch räumlich einschränkende Wände und hydrodynamische Flussfelder zu manipulieren. Um das mechanische Verhalten der Biopolymere zu analysieren und zeitlich und räumlich aufgelöste Reaktionen zu beobachten, kann die Mikrofluidik sehr gut mit an die experimentellen Anforderungen angepassten Untersuchungsmethoden wie Fluoreszenzmikroskopie, Polarisationsmikroskopie und Röntgenstreuungssanalyse kombiniert werden.

Die Analyse einzelner fluktuierender Aktinfilamente, deren Bewegung durch enge Kanäle beeinträchtigt ist, ermöglicht eine umfassende Beschreibung der mechanischen Systemeigenschaften. Untersuchungen mit Hilfe der Fluoreszenzmikroskopie zeigen, dass das Verhalten der Biopolymere von ihrer Konturlänge $L$ und der Umgebung, also der Form und Abmessungen der Kanäle, abhängt. Der äußere Einfluss wird als ein parabolisches Wandpotential angenommen. So gelingt es, den Wettstreit zwischen der Biegeenergie der Filamente und dem Einfluss der Kanalwände bemerkenswert genau zu beschreiben. Die Ergebnisse der Experimente sind außerdem im Einklang mit Monte-Carlo-Simulationen und mit Skalengesetzen für die Abstoßlänge $\lambda$ und für die Segmentverteilung in Kanälen.

Die Experimente an Kollagen I bieten tiefe Einblicke in die dynamische Entwicklung der hierarchischen Organisation natürlicher Kollagenfibrillen. Wir verwenden 
hydrodynamische Fokussierung in Verbindung mit diffusivem Materialtransport um einen stabilen pH-Gradienten in der Mikrofluidikumgebung zu erzeugen. Dadurch sind wir in der Lage, Nichtgleichgewichtsmessungen durchzuführen und verschiedene Stadien des Selbstorganisationsprozesses an verschiedenen Positionen entlang der Reaktionskoordinate zu beobachten. Wir untersuchen das System auf der Mikrometerskala, indem wir den Vorteil nutzen, dass Kollagen doppelbrechend ist. Zusätzlich können wir mit Röntgenstreuungsanalyse die dynamische Entstehung der kritischen Untereinheiten der Kollagenfibrillen auf der Nanometerskala beobachten. Ein detaillierter Vergleich mit Simulationen zeigt, dass die Finite-Elemente-Methode eine gute Beschreibung unserer experimentellen Ergebnisse im Bezug auf Diffusionsphänomene, pH-Verteilung und den Einfluss der Lösungsviskosität auf das Flussprofil liefert.

Die hier vorgestellten Experimente bringen das fundamentale Verständnis der untersuchten biologischen Systeme voran und zeigen darüber hinaus das Potential mikrofluidischer Techniken auf, umfassende Bereiche der Lebenswissenschaften weiter zu entwickeln. 


\section{Contents}

$\begin{array}{lll}1 & \text { Introduction } & 1\end{array}$

2 State of the Art 5

2.1 Actin Research . . . . . . . . . . . . . . . . . . . 5

2.1 .1 Biological Relevance . . . . . . . . . . . . . 5

2.1 .2 Microfluidics and Life Science . . . . . . . . . . . . . . 7

2.1.3 Actin as a Model System for Semiflexible Polymers . . . . . . 8

2.2 Collagen Research . . . . . . . . . . . . . . . . . . . . . . . . 10

2.2 .1 Biological Relevance . . . . . . . . . . . . . . 10

2.2 .2 Implications for Tissue Engineering . . . . . . . . . . . . . . . 14

$\begin{array}{lll}3 & \text { Materials and Methods } & 15\end{array}$

3.1 Biological Systems . . . . . . . . . . . . . . . . . . . 15

3.1.1 Molecular Mechanisms of Actin Polymerization . . . . . . . . 15

3.1.2 In Vitro Actin Polymerization . . . . . . . . . . . . . . . . . . 17

3.1.3 Collagen Characterization . . . . . . . . . . . . . 18

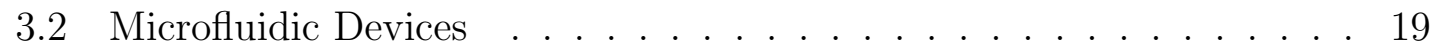

3.2 .1 Soft Lithography . . . . . . . . . . . . . . . . . . . . . 19

$3.2 .2 \quad$ Assembly of Microfluidic Flow Chambers . . . . . . . . . . . . 21

3.3 Fluorescence Microscopy . . . . . . . . . . . . . . . . . . . . . . . 25

3.4 Polarized Light Microscopy . . . . . . . . . . . . . . . . . . . . . . . . 27

3.5 X-Ray Microdiffraction . . . . . . . . . . . . . . . . . . . . 28

3.6 Software . . . . . . . . . . . . . . . . . . . . . . . . . . . 29

vii 
4 Worm-Like Chain Model 31

4.1 Definition of the Persistence Length . . . . . . . . . . . . . . . . . 31

4.2 Unconfined Polymers . . . . . . . . . . . . . . . . . . . . . . 32

4.2 .1 Tangent Correlation Function . . . . . . . . . . . . . . . . 32

4.2 .2 Radial Distribution Function . . . . . . . . . . . . . . . . . . . 33

4.3 Confined Polymers . . . . . . . . . . . . . . . . . . 34

4.3 .1 Tangent Correlation Function . . . . . . . . . . . . . . . 34

4.3 .2 Radial Distribution Function . . . . . . . . . . . . . . . 38

$4.3 .3 \quad$ Segment Distribution . . . . . . . . . . . . . . . . . . 38

5 F-Actin in Microfluidic Environment 41

5.1 Image Processing and Data Analysis . . . . . . . . . . . . . . . . . 41

5.2 From Free to Confined Filaments . . . . . . . . . . . . . . . . . . 46

5.2.1 Characterization of Unconfined Filaments . . . . . . . . . . . 46

5.2.2 Tangent Correlation Function of Confined Filaments . . . . . 47

5.2 .3 Influence of Contour Length and Channel Width . . . . . . . 50

5.3 Radial Distribution Function . . . . . . . . . . . . . . . . . . 57

5.4 Segment Distribution in the Microchannels . . . . . . . . . . . . . . . 61

5.5 Additional Bending of Confined Filaments . . . . . . . . . . . . . . . 63

$5.6 \quad$ Future Directions . . . . . . . . . . . . . . . . . . . . 67

$\begin{array}{lll}6 & \text { In Situ Collagen Self-Assembly } & 69\end{array}$

6.1 Hydrodynamic Focusing and Diffusive Mixing . . . . . . . . . . . . . 69

6.2 Physics of the Microflow . . . . . . . . . . . . . . . . . . 72

6.3 The Finite Element Method . . . . . . . . . . . . . . . . . . . . 74

6.4 Hydrodynamics in Microchannels . . . . . . . . . . . . . . . 75

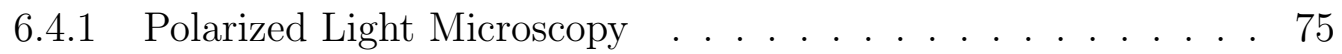

6.4 .2 Influence of Solution Viscosity . . . . . . . . . . . . . . . 76

6.5 Collagen in $\mathrm{pH}$ Gradient . . . . . . . . . . . . . . . . . . . 80

6.5.1 Diffusive Phenomena . . . . . . . . . . . . . . . . 80 
6.5 .2 Calculation of the $\mathrm{pH} \ldots \ldots$. . . . . . . . . 81

6.5.3 Comparison of Experiment and Simulation . . . . . . . . . . 85

6.6 X-Ray Microdiffraction . . . . . . . . . . . . . . . . . . . . . 88

6.7 Future Directions . . . . . . . . . . . . . . . . . . . . . . 93

$\begin{array}{lll}7 & \text { Conclusions } & 95\end{array}$

\begin{tabular}{|lr}
\hline A List of Abbreviations & 99
\end{tabular}

\begin{tabular}{lc}
\hline Bibliography & 103
\end{tabular}

\begin{tabular}{ll}
\hline Acknowledgements & 115
\end{tabular}

$\begin{array}{lr}\text { Curriculum Vitae } & 117\end{array}$ 


\section{Chapter 1}

\section{Introduction}

In 1943 Erwin Schrödinger raised the question "What is life?" which was the title of his well-known book and can in some respect be considered as the initiation of biophysics.[1] Up to present time the interest of scientists in the physical properties of biological systems is undiminished - to a considerable extent certainly because there are still numerous unanswered questions and unresolved problems. The human body consists of roughly $10^{14}$ cells all of which serve their unique function and meet very specific requirements. One of the most fascinating phenomena in biology is that while every individual cell is a very complex and sophisticated organism itself, the ensemble of all cells reveals a unique team play which eventually results in the remarkable functionality of every multi-cellular organism.

Besides biochemical signaling mechanical phenomena play a key role for cellular processes. The physics of the cell as well as of its surrounding are to a great extent influenced by the properties of fibrous biopolymers. Cell motility, division, shape and mechanical stability of eukaryotes are mainly determined by the cytoskeleton, a flexible scaffold made up of a set of fibrous proteins - microtubules, intermediate filaments, and actin. They all fulfill very specific tasks in the cell to which their structure and mechanical properties are perfectly adapted. Microtubules are stiff, hollow cylinders with a diameter of around $25 \mathrm{~nm}$ and are mainly responsible for intercellular transport. Intermediate filaments are ropelike fibers with a diameter of about $10 \mathrm{~nm}$ which basically provide the cell with mechanical strength. Actin filaments have a diameter of about $8 \mathrm{~nm}$ and govern, among other things, the cell's motility and shape. Thus, the mechanical and dynamic properties of cytoskeletal proteins and their supramolecular organization are important issues whenever living beings are studied. Cells are embedded in the so-called extra cellular matrix (ECM) whose main component is the protein collagen I. Collagen I is highly hierarchically 


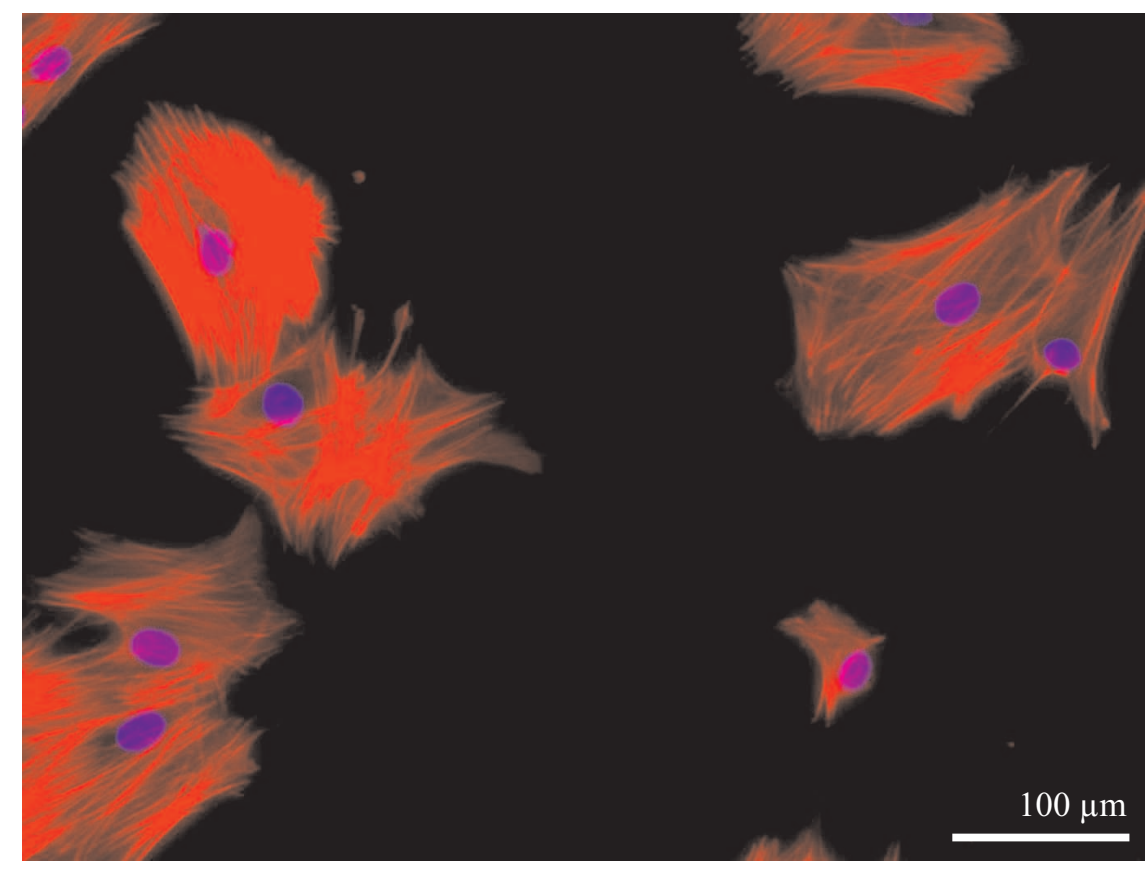

Figure 1.1: Fluorescence micrograph of vascular smooth muscle cells. The actin cytoskeleton is stained red, the DNA in the nuclei is stained blue. In vivo, the cells are embedded in a matrix of fibrous proteins, mainly collagen and elastin.

organized on the nano-, micro-, and macroscale - a structural property which also effects the functionality of tissue. In addition to investigations of the intra- and extracellular polymer networks and their components themselves, interactions communicated via the cell membrane are of great scientific interest. One example for such interactions is the muscle layer in blood vessels as shown in figure 1.2, where muscle cells and collagen fibrils are circumferentially organized and influence each others alignment. $[3,4]$

In recent years, microfluidic methods have in many respects revolutionized biophysical, biochemical and biomedical research. Microfluidic techniques play an ever greater role for the development of so-called labs on a chip or $\mu$ TAS (total analysis systems) since the rapid and efficient analysis of even minimal sample volumes requires tools for the handling of simple as well as complex fluids. As soon as biopolymers are involved in the reactions and processes which take place on microfluidic chips a principle understanding of the fundamental behavior of these molecules under geometric confinement is indispensable. In addition to these applications, devices containing channels on the micrometer scale provide excellent means to study biological macromolecules and even whole cells in an environment which is simplified enough to describe it theoretically but yet includes the basic features of the corresponding in vivo system. We can thus gain a deeper fundamental understanding of cellular and biopolymer mechanics. 

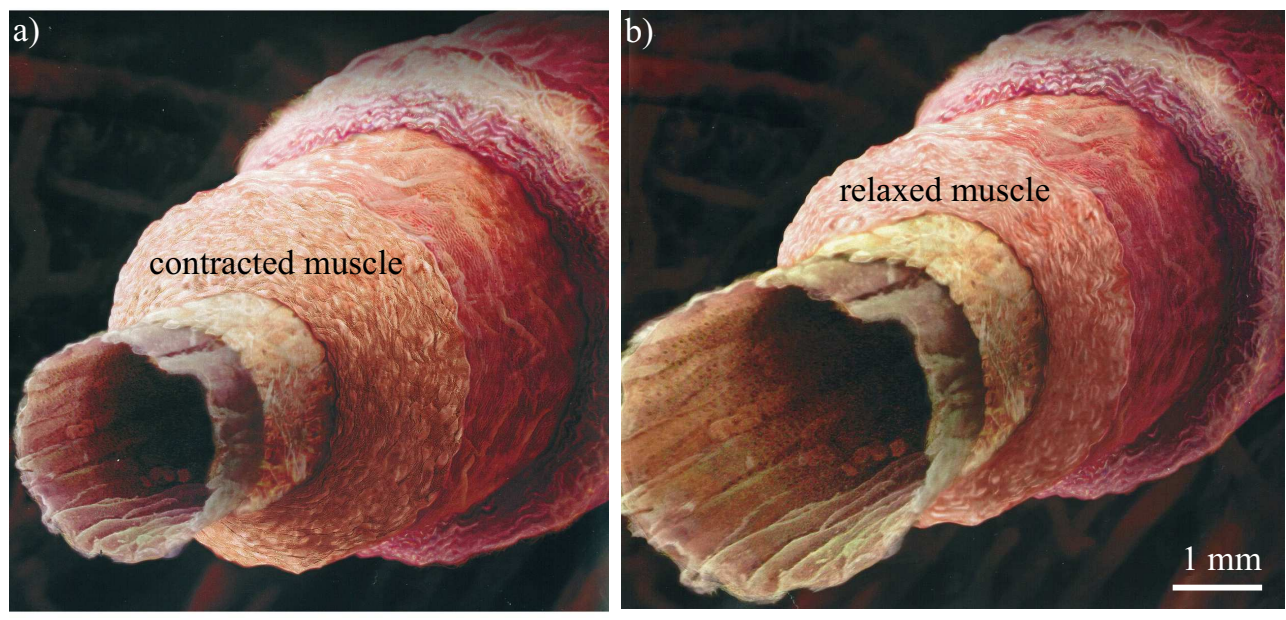

Figure 1.2: Illustration of the layering structure of a blood vessel. The vessels fine tune the blood pressure by slow contraction (a) and relaxation (b).[2] The diameter of blood vessels ranges between $0.01 \mathrm{~mm}$ and $3 \mathrm{~cm}$.

Here, we present studies of two different biological systems, the cytoskeletal protein actin and the extracellular matrix protein collagen I. Both systems provide fundamental insights into tissue and cellular mechanics and functionality since they represent very abundant and hence important building blocks of intra- and extracellular protein networks. In chapter 2 , the biological relevance of the studied systems and the state of the art in theoretical and experimental polymer research, microfluidics, and tissue engineering will be discussed. Materials and methods will be described in chapter 3. In chapter 4, the theoretical background that is relevant for the description of semiflexible polymers will be presented. The results concerning experiments on single actin filaments in microchannels will be discussed in chapter 5 followed by those concerning the study of hierarchical organization of collagen I in chapter 6 . 


\section{Chapter 2}

\section{State of the Art}

In this chapter we will relate our work to a broader context of current research by discussing the biological relevance of the studied systems and by describing microfluidics as a tool for biophysical and biochemical investigations. Furthermore, we will motivate our choice of projects by identifying some open questions concerning the state of the art in both actin and collagen research.

\section{$2.1 \quad$ Actin Research}

\subsubsection{Biological Relevance}

The cytoskeleton is responsible for the motility, shape and structural support of the cell. Actin is the most abundant intracellular protein - in muscle cells it amounts to $10 \%$ by weight of the total cell protein and even in non-muscle cells it constitutes $1-5 \%$. Consequently, actin plays a key role in many cellular processes. This assumption is also supported by the fact that actin is highly conserved - only very few differences between yeast and human actin could be detected. Because such a great variety of critical functions is related to actin, minor changes would have the potential to interfere with many crucial processes. Globular actin monomers (G-actin) are moderate sized having a molecular weight of $42 \mathrm{kDa}$. Each monomer contains an ATP (adenosine triphosphate) binding cleft and is stabilized against denaturing by the binding of ATP. A two-stranded helical filament (F-actin) with a diameter of about $8 \mathrm{~nm}$ and a helical pitch of $37 \mathrm{~nm}$ is assembled from G-actin. The resulting filamentous macromolecule displays a characteristic polarity: At the so-called "minus-" or "pointed" end the ATP binding cleft is exposed to solution, whereas at the "plus-" or "barbed" end it contacts the neighboring actin-subunit. 
Some of the cellular processes where actin is involved will be briefly discussed in the following section.

The shape of a cell is mainly determined by the actin network which is attached to the plasma membrane by other proteins. In fact, most actin in a cell lies directly beneath the membrane and is arranged in networks and bundles. Self-generated cell movement (motility) and force generation (for example muscle cell contraction) are mainly caused by two mechanisms both involving actin: the assembly and disassembly of actin filaments and the molecular motor system composed of actin and myosin. The high flexibility of the cytoskeleton is due to different and varying filament lengths and manifold actin binding proteins (ABPs). Regulated (de-)polymerization of actin filaments leads to dramatic changes in cell shape as can be seen in platelets during blood clotting, for example. The variety of ABPs is extensive. Some of these molecules, like fimbrin, $\alpha$-actinin, spectrin, and filamin, bind several actin filaments together and thus stabilize actin networks and bundles. Other ABPs regulate actin polymerization and filament length. While in vitro polymerization can be initiated by addition of salts, cells have to maintain constant ionic strength. Therefore, other mechanism are employed, namely actin polymerization is regulated by $\mathrm{G}$-actin binding proteins such as thymosin $\beta_{4}$ or profilin. Different toxins inhibit polymerization (such as cytochalasin D, which binds to the plus end of F-actin, or latrunculin, which binds to G-actin) or depolymerization (such as phalloidin, the poison of the "angle of death" mushroom, which locks adjacent monomers together). Severing proteins break filaments apart (for example gelsolin or cofilin), and capping proteins stabilize F-actin (for example CapZ or tropomodulin). These mechanisms are needed in places where the organization is desired to remain unchanged, such as in muscles or erythrocytes.

Cell migration is probably the most prominent process where reorganization of actin filaments plays a crucial role. In this case, actin polymerization occurs at the leading edge of a moving cell. Another example where impressive forces are generated is the interaction between myosin II and actin involved in muscle contraction and cytokinesis. The so-called sarcomere consists of a repeating structure of myosin thick filaments and actin thin filaments. It is contracted when myosin heads walk along F-actin. Myosin II forms dimers of two heavy chains, whose tail domains associate to form thick bundles. This structure enables the head domains to interact simultaneously with actin thin filaments. The movement is performed in discrete steps of $5-10 \mathrm{~nm}$ and produces a force of $3-5 \mathrm{pN}$ per molecule. Additionally, there are many other forms of myosins which have different functions in the cell. [3, 4] Since actin is involved in so many different processes, it has become a very popular object 
of research and a fundamental understanding of the characteristics and properties of this protein is highly desirable.

\subsubsection{Microfluidics and Life Science}

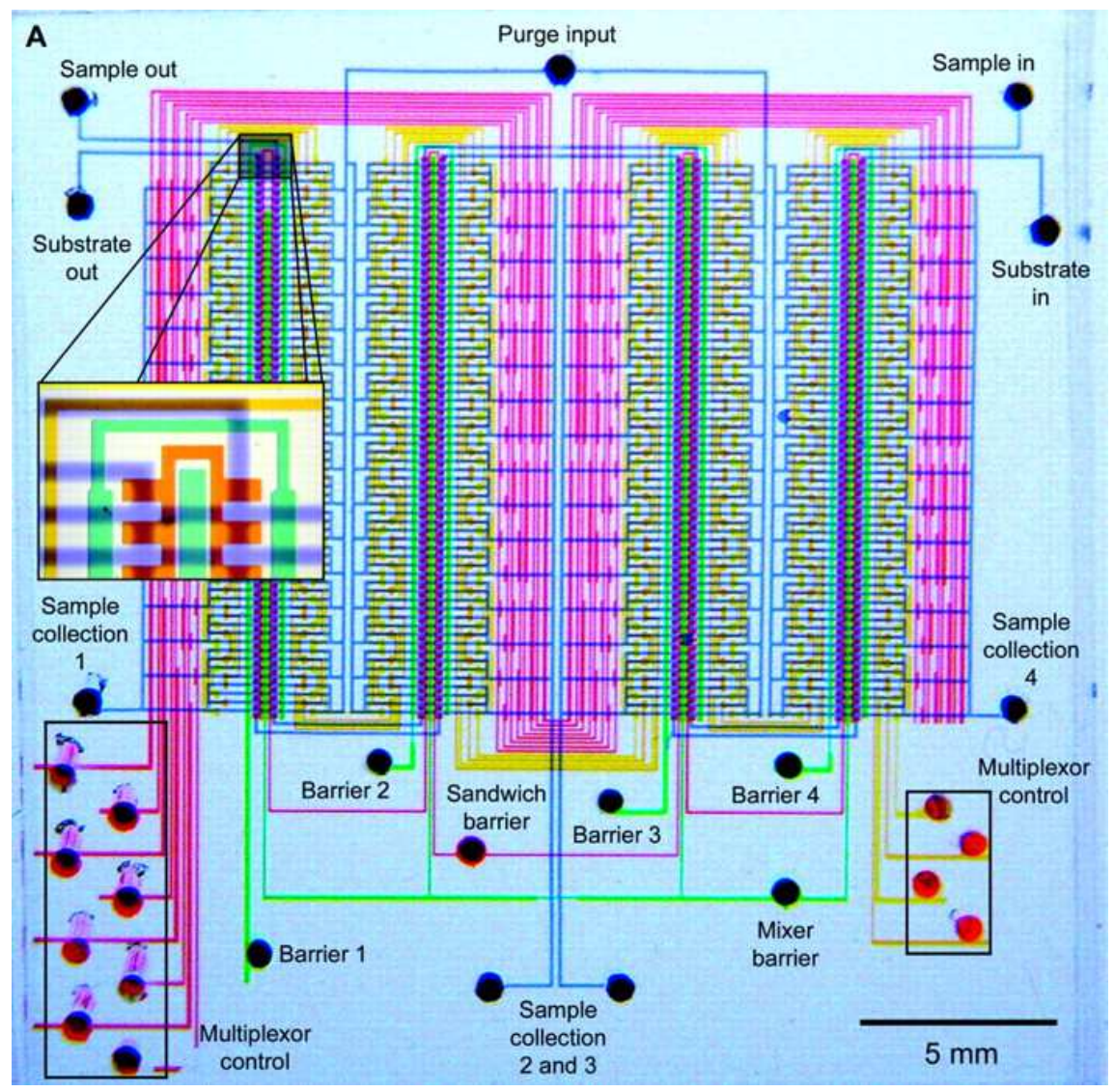

Figure 2.1: Optical micrograph of a microfluidic chip. The various inputs have been loaded with food dyes to visualize the channels and subelements in the fluidic logic.[5]

In recent years, microfluidics has been discovered as an excellent tool to study biological and biochemical systems. New techniques allow for rapid and efficient handling and analysis of ever smaller sample volumes. This is especially important since certain substances like proteins are expensive, difficult to purify, or - as in the case of forensic DNA material - simply not available in large amounts. For these reasons significant research efforts have been devoted to developing so-called "micro-labs" on fluidic chips. The use of channel networks on the micrometer scale implies that a principle understanding of the influence of confining geometries, hydrodynamic flow, and stress on biological systems becomes increasingly important for the design of microfluidic applications in lab-on-a-chip platforms. [6-9]

In order to gain a better understanding of so-called "complex fluids", such as polymer 
solutions, under the influence of confining microfluidic devices, several groups have studied single macromolecules in microchannels. The most popular system for such experiments is lambda bacteriophage DNA ( $\lambda$-DNA) because the single molecules are rather stable to breakage and the long (48.5 kilo base pairs, which corresponds to $16.5 \mu \mathrm{m}$ ) monodisperse strands can easily be fluorescently stained using intercalating dyes. Moreover, the system is well characterized in literature. Pioneering experiments where single DNA strands have been exposed to elongational flow [10] have been followed by further studies of the influence of flow on chain molecules with special attention to stretching and successive relaxation.[11,12] The transition to nanometer sized channels, which then are in the same order of magnitude as the persistence length of the DNA chains, has led to the conclusion that the crossover from the flexible ("De Gennes") regime [13] to the semiflexible ("Odijk") regime [14] takes place at a confining length scale roughly twice the persistence length of the molecules.[7]

Microfluidics provides a very controlled environment to conduct such experiments since the channel dimensions are comparable to the contour lengths of the molecules and exterior parameters such as confinement and hydrodynamic flow can be defined in a straight forward manner. Even whole cells can be studied in a microfluidic device, each individuum encapsulated in a droplet.[15] This provides detailed characterization of the individual biological system. Furthermore, accurate statistical information can be obtained by screening a large pool of individual molecules or cells. Moreover, systems of channels on the microscale provide an excellent method to mimic certain confining biological systems such as blood vessels or protein networks. One prominent example of such physiological confinement is the dense cytoskeletal network which has a mesh size on the order of micrometers and is exposed to permanent structural changes. It is therefore of substantial interest to investigate the individual macromolecule in an environment that resembles its native surroundings. $[16-18]$

\subsubsection{Actin as a Model System for Semiflexible Polymers}

The physical properties of biopolymers are very challenging to investigate in vivo and the poor understanding of the mechanical details of the cytoskeleton further complicates a theoretical description of these systems. [19,20] Therefore, extensive research effort has been devoted to modelling the cellular mechanics with purified, reconstituted in vitro systems. Actin takes an exceptional position among the cytoskeletal biopolymers. In addition to its considerable importance in life science (see 
section 2.1.1), it also serves as one of the few experimentally accessible model systems for semiflexible chain polymers.[21,22] Fortunately, actin can quite readily be polymerized in vitro and experiments can be conducted in cell-free systems. Since the contour length $L$ of the filaments is on the order of several micrometers the fluorescently labeled biopolymers can be observed by optical microscopy. A crucial measure in polymer science is the persistence length $L_{P}$. Generally speaking, it is the arc length distance along the contour of the molecule at which two polymer segments have lost any correlation. In recent years, several experimental studies on freely fluctuating actin filaments in dilute solutions have been performed, elucidating some of the mechanical and statistical properties of these biopolymers. In the past, different methods have been employed to determine the persistence length of actin filaments. All of these studies restricted the fluctuations of the biopolymers to two dimensions by confining the molecules between glass slides with distance $\sim 1-6 \mu \mathrm{m}$ or keeping them in the focal plane of the microscope using optical tweezers. The persistence length of the biopolymers has been deduced from the mean square endto-end distance, [23] from the Fourier modes of the fluctuation, [24, 25] the heightheight correlations, [26] the tangent correlation function, $[21,22,26]$ the reaction of the filaments to external forces [27] and the radial distribution function. [21] These experiments revealed a persistence length of $7.4-22 \mu \mathrm{m}$. As this length scale is in the same order of magnitude as the contour length, F-actin is a semiflexible polymer and is situated between stiff biopolymers and flexible biopolymers. Therefore, actin can serve as a model system not only for semiflexible but also for more flexible and stiffer polymers once the contour length is adjusted appropriately.

A different approach has been followed by studying more concentrated actin solutions and the interactions of actin and other molecules. Outstanding experiments in this field are the investigation of self-assembly in the presence of lipids, [28] actin- $\alpha$ actinin network formation with [29] and without [30] confining microchannels, actin bundling in the presence of multivalent counter-ions, [31] studies of the mechanical properties of actin networks and gels, $[32,33]$ and "artificial muscles" composed of actin and myosin. [34]

The theoretical description of semiflexible polymers has also advanced to a great extent in recent years. The radial distribution function has been determined for free [35] and confined [36] filaments. Also, polymers confined in soft tubes,[37] in a parabolic potential,[38] in rectangular tubes,[39] and in cylindrical pores [14,40] have been discussed. The free energy of confined polymers has been determined.[39,41,42] More concentrated solutions of actin, [43] the reptation motion of single actin filaments in semidilute F-actin solutions, and bundling of individual filaments [44] has 
also been described.

However, studies on individual actin filaments in an environment which mimics their natural surrounding in terms of the degree of confinement but is yet simple enough to be theoretically describable are largely missing. In this thesis, we will therefore present characterization of the Brownian motion of F-actin confined in microchannels. $[45,46]$

\subsection{Collagen Research}

\subsubsection{Biological Relevance}

b)

a)

c)

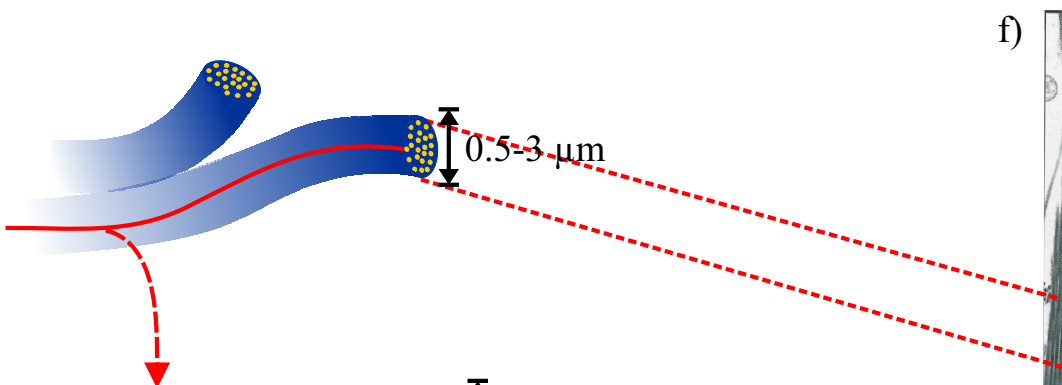

f)

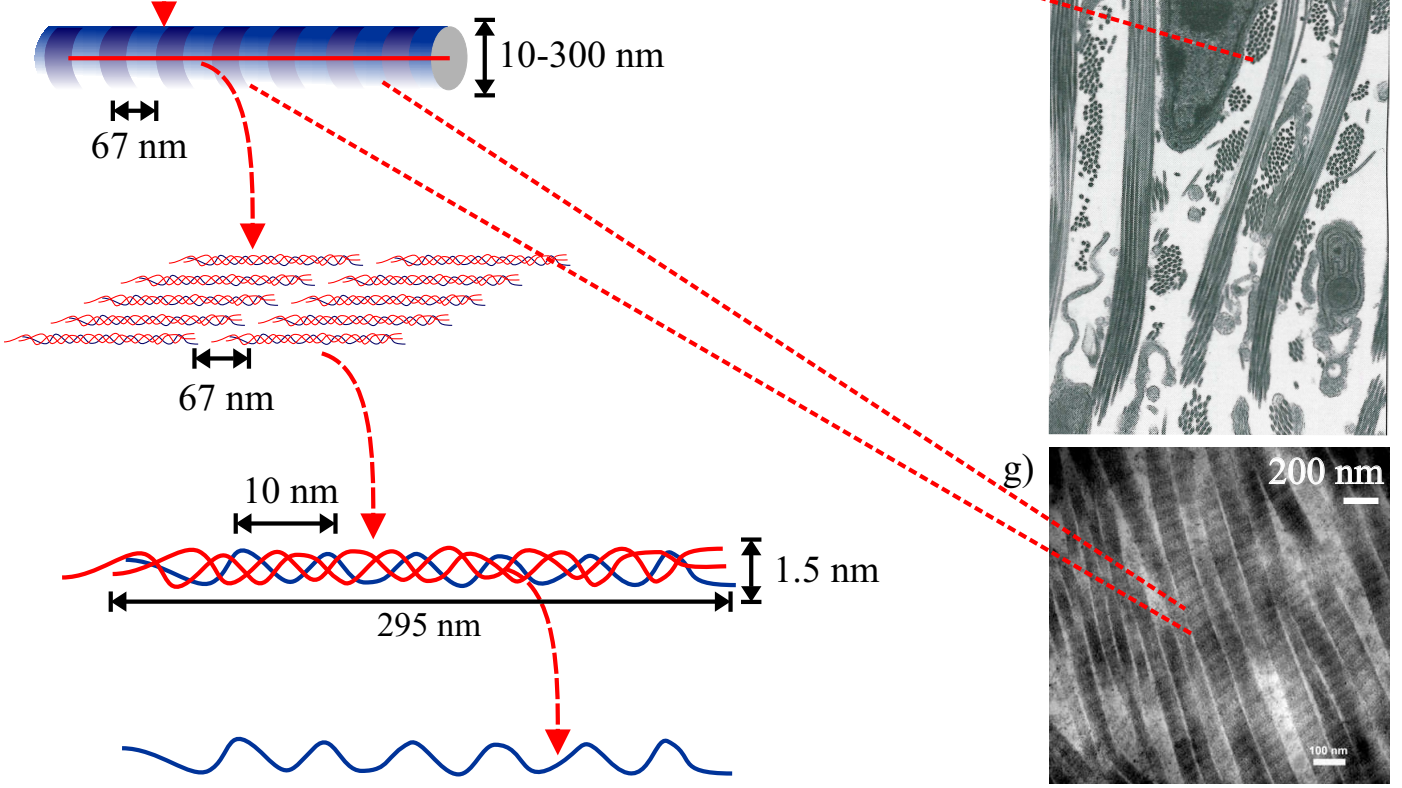

Figure 2.2: The hierarchical organization of collagen. a - e) Schematic representation: a) Collagen fiber consisting of b) collagen fibrils. The characteristic striation stems from the staggered organization of c) collagen subunits. d) Helical collagen monomers consist of e) three peptide chains each (two $\alpha_{1}$, one $\alpha_{2}$ chains). f) Electron micrograph of a fibroblast surrounded by collagen fibrils in the connective tissue of embryonic chick skin.[47] g) Electron micrograph of characteristically striated collagen fibrils.[48] 


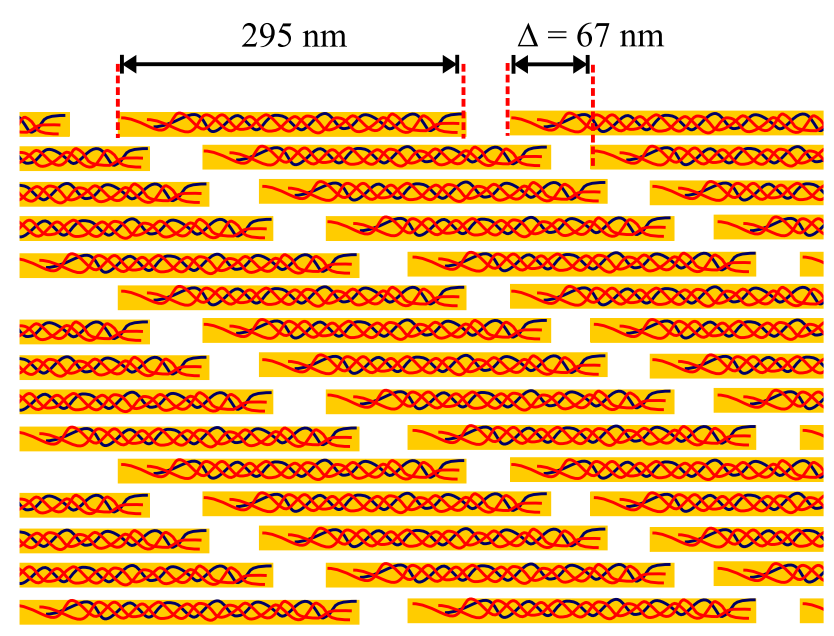

Figure 2.3: Model for two dimensional aggregate of collagen molecules. The triple helical molecules are laterally displaced by $\Delta$.

Collagens are a family of fibrous proteins which are found in all multicellular organisms. They are the major component of bone and skin and represent about $25 \%$ of the total protein mass in animals. More than 20 different types have been identified and classified primarily according to their physiological structure. Fibrillar or fibril-forming collagens form higher order polymers. Fibril-associated collagens decorate the surface of collagen fibrils and link them together or to other molecules. Network-forming collagens assemble into a feltlike sheet or meshwork. They constitute a major part of the mature basal lamina, which is the layer on which epithelium sits. Here, we will focus on collagen I, which represents $90 \%$ of the total body collagen and belongs to the group of fibril-forming proteins. The macroscale structure and organization of collagen I fibrils are key contributors to the mechanical properties of soft tissue, bone, tendon, and ligaments. Furthermore, the organization of collagen on the microscale profoundly influences cell morphology, migration, proliferation and gene expression.[49]

Collagen is synthesized in the endoplasmatic reticulum of fibroblasts and other cells. The structure of the peptide chains is extremely repetitive: Every third position is occupied by glycine, which is the smallest amino acid and can be packed tightly inside the triple-stranded helical structure characteristic of collagen. Another $22 \%$ of the amino acid sequence is constituted by proline which stabilizes the helical structure. The disadvantage of this highly repetitive structure is that denatured collagen, referred to as gelatine, cannot return easily to its original state once the triple helices are broken apart. In the cell, signal peptides are added to both ends of the collagen peptide chain. The propeptides at the C-terminus are bound via disulfate groups and facilitate the formation of the triple helix.[50] The helical pitch 
a)

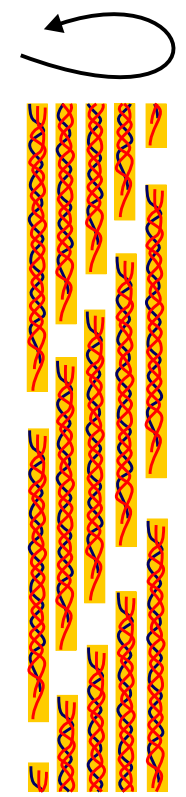

b)

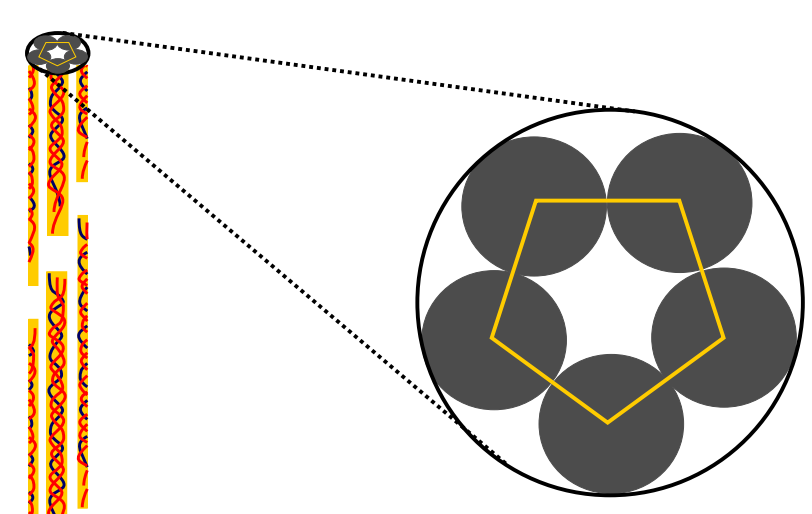

Figure 2.4: Schematic representation of the model for three-dimensional arrangement of collagen molecules in five stranded $\Delta$ periodic microfibrils. a) The minimum number of laterally assembled molecules is five. b) In order to transfer the model to three dimensions, cylinders have to be formed. c) Top view of the pentameric cylinders.

is $10 \mathrm{~nm}$ and the diameter is $1.5 \mathrm{~nm}$. An advantage of the additional non triple helical groups at the $\mathrm{C}$ - and N-termini of the peptide chains is that premature fibril formation inside the cell is inhibited. The resulting procollagen molecules, a precursor form of collagen, are secreted from the cell. In the extracellular space, the collagen molecules hierarchically self-assemble at the nano-, micro-, and macroscales into many different structures which are adapted to their physiological function.

A simplified representation of the principle hierarchical stages is shown in figure 2.2 . Already inside the cell single collagen peptide chains assemble into triple stranded helical structures. These procollagen molecules, also referred to as collagen monomers, self-assemble into critical subunits. The non triple helical regions (propeptides) at the C- and N-termini of the procollagen molecules play an important role in collagen self-assembly. In the presence of these propeptides lateral self-assembly is limited to five molecules. [51] The molecules self-assemble linearly to form $4 \mathrm{~nm}$ diameter fibril subunits. [52] Only upon cleavage of the propeptides does a further lateral assembly into thicker fibrils and eventually fibers occur. Collagen molecules without propeptides are called tropocollagen. Collagen fibrils show a characteristic striation in electron micrographs (see figure $2.2 \mathrm{~g}$ ) since the collagen monomers are organized in a staggered manner with a period comprising of gap and overlap regions.[53] X-ray diffraction studies have shown that in the hydrated state the period 
is $\Delta=67 \mathrm{~nm} .[54]$

While the linear periodicity is rather easy to detect by electron microscopy much more effort has to be devoted to the investigation of the lateral packing. Especially the lateral organization of the critical subunits remains somewhat unresolved [55] although theoretical models and X-ray data exist. In 1968 the existence of a five stranded microfibril has been deduced from the $\Delta$-periodicity parallel to the axis of the collagen fibrils.[56] The length of a triple helical molecule is $295 \mathrm{~nm}=4.4 \Delta$. The dark and light regions which are observable in collagen fibrils by electron microscopy upon negative staining (heavy metal stains are incorporated in the fibril, highlighting predominantly the gap regions between two adjacent molecules) suggest that the molecules are laterally displaced by $\Delta$ with an overlap region of $0.4 \Delta$, leaving a gap of $0.6 \Delta$. A schematic representation of this arrangement is shown in figure 2.3. In order to transfer this two-dimensional model to three dimensions, hollow cylinders have to be formed from the sheets. The minimum number of molecules needed to form such a cylinder with optimized molecular packing is five. This leads to the arrangement of collagen molecules in five-stranded $\Delta$-periodic microfibrils (figure 2.4). This basic model has been further refined. It has been suggested that upon further lateral compression the pentameric subunits disappear and a quasi hexagonal lattice forms. [54,57] The theoretical model has been supported by subsequent X-ray diffraction data. [58,59] Interestingly, the formation of a $4 \mathrm{~nm}$ microfibril as the critical subunit of collagen self-assembly has been observed in isolated in vivo systems, for example in the cornea [60] or in rat tail tendon [58] as well as in in vitro systems, as shown for precipitated collagen aggregates by electron microscopy.[61] However, to our knowledge there is no study which uses X-ray diffraction to resolve the molecular packing of in vitro assembled collagen.

With respect to mechanical properties, the alignment of collagen fibrils plays a clear role: By aligning in the direction of highest tissue strain, the collagen fibrils act as reinforcement to the tissue structure.[62] Aligned collagen fibers can also guide cell migration through contact guidance. [63] To this end, much research effort has been devoted to preparing aligned collagen networks through the use of intense magnetic [64,65] or electric fields, cell-generated traction forces, [65] hydrodynamic flow, [66] dip-pen nanolithography, [67] and interfacial orientation on the surface of other biopolymers. [68] However, most of these methods lack microscale control of the self-assembly process, and none are amenable to dynamic investigations of the self-assembly process. We are particularly interested in controlling structural properties such as the degree of alignment and concurrently investigating the dynamics of the assembly process in situ. 


\subsubsection{Implications for Tissue Engineering}

Countless tissue replacements are needed for medical applications every year: for burn patients, patients with cardiovascular diseases, or patients in need of bone replacements. Up to now, physicians are often bound to the use of synthetic materials such as titanium and cobalt alloys, ceramics and polyethylene for hip joint prostheses, and Dacron ${ }^{\circledR}$ or Goretex ${ }^{\circledR}$ for vessel replacements. Alternatively, it would be highly desirable to use natural materials with higher biological tolerance, or at least biodegradable scaffolds which sustain the newly developing material and degrade after a certain time.

Since collagen is the most abundant protein in the animal kingdom, it also plays a very important role for engineered functional tissue replacements such as extracellular matrices for the repair of orthopedic soft tissues, [69] heart valves, [70] and blood vessel replacements. [71] In many cases, anisotropic, aligned, or structured collagen networks or gels, or substrates modified with collagen provide the basis for such engineered tissues. [72] These materials have therefore been used extensively to investigate cell behavior.[63,73,74] In vitro, the collagen triplehelical monomers are stable at acidic $\mathrm{pH}$ and assemble into fibrils at $\sim \mathrm{pH} 6$. This process is often referred to as "fibrillogenesis" [75] or "gelation". [61] While the pH dependence of collagen fibrillogenesis is known in principle, $[61,75]$ its understanding is complicated by the fact that existing methods cannot precisely predict the $\mathrm{pH}$ within the system. However, these $\mathrm{pH}$ conditions are crucial for the comprehension of the process of collagen assembly into its unique hierarchical organization. The mechanical properties of protein matrices have also been extensively investigated since they are a key factor for tissue engineering. [76, 77] 


\section{Chapter 3}

\section{Materials and Methods}

The utilization of biological matter in vitro requires specific handling. The experimental techniques along with the methods of measurement will be described in detail in this chapter.

\subsection{Biological Systems}

\subsubsection{Molecular Mechanisms of Actin Polymerization}

Fortunately, actin can be polymerized in vitro. The resulting filaments cannot be distinguished from those isolated from cells. [3] In many cases, it is easier to perform experiments in a cell-free environment as compared to the much more complex in vivo system since the number of parameters which have to be controlled and understood is smaller. Certainly, actin owes its popularity amongst experimental polymer scientists and biophysicists partly to this excellent in vitro manageability. The process of actin polymerization in vitro can be divided into three steps (figure 3.1). The nucleation step leads to a stable aggregation (blue in figure 3.1) of three to four actin monomers. The globular actin monomers have a diameter of about $8 \mathrm{~nm}$, and a molecular weight of $42 \mathrm{kDa}$. The number of amino acids can be estimated to 382 assuming 110 Da per amino acid. Formation of a nucleus occurs relatively rarely since a third monomer necessarily has to interact with the complex of two monomers before they separate again. During the following growth phase ATP-Gactin (adenosine triphosphate globular actin, gray in figure 3.1) is successively added to the existing filament with a rate constant $k_{o n}$. The ATP is slowly hydrolyzed to ADP (adenosine diphosphate) and stable ADP-F-actin (filamentous actin, red in figure 3.1) forms. Simultaneously, depolymerization occurs with a rate constant 


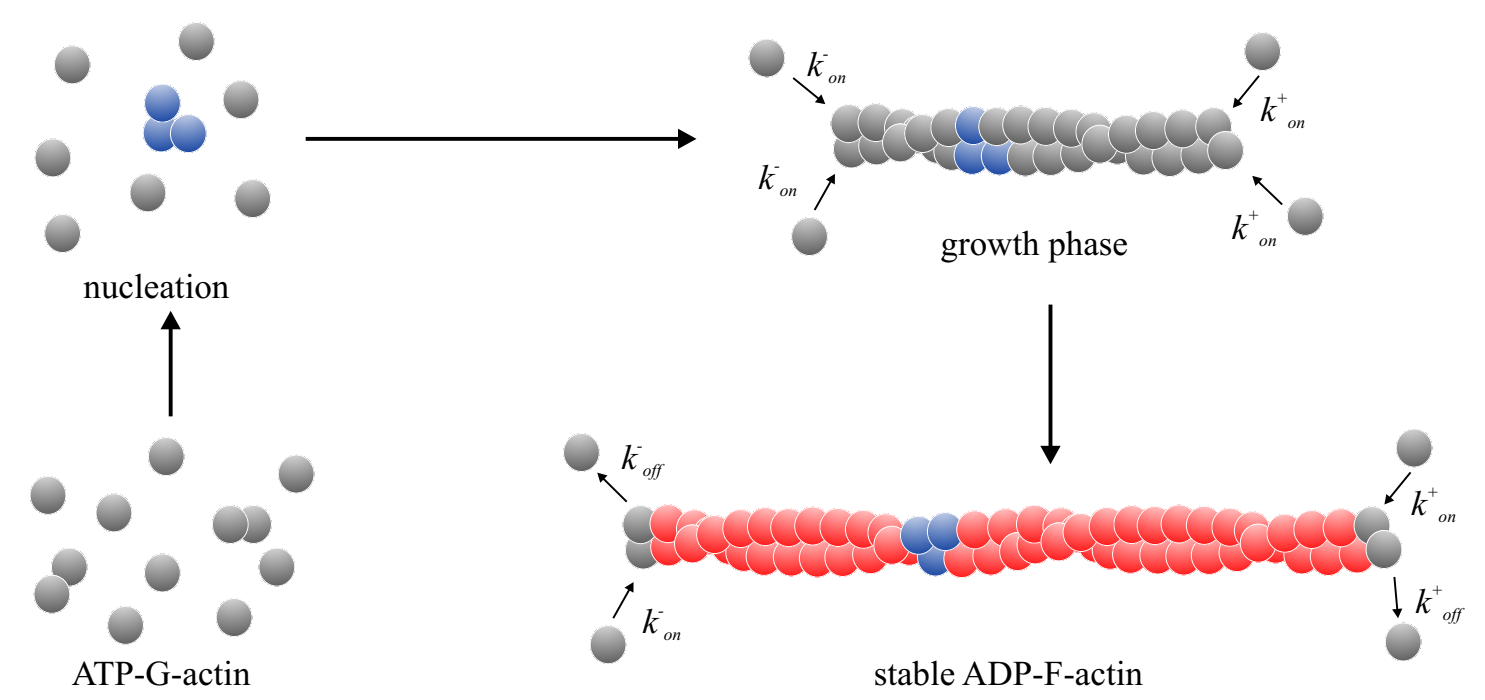

Figure 3.1: Actin polymerization is a three step process including nucleation, growth phase, and steady-state. Stable nuclei (blue) form and are elongated by the addition of ATP-G-actin (gray). Stable ADP-F-actin (red) results from ATP hydrolysis.

$k_{o f f}$. The system is in steady-state when the rate of ATP-G-actin addition equals the rate of ADP-G-actin loss. The corresponding G-actin concentration is the critical concentration $c_{c}$, which correlates the rate constants $k_{o n}$ and $k_{\text {off }}$

$$
k_{\text {off }}=c_{c} k_{o n} .
$$

This indicates that the amount of added ATP-G-actin depends on the concentration in the solution, but not on the amount of removed ADP-G-actin. $c_{c}$ depends very much on the conditions in the actin solution such as temperature and ion concentration. At $4{ }^{\circ} \mathrm{C}$ and in the absence of monovalent and divalent ions $c_{c}$ is more than $3 \mathrm{mg} / \mathrm{mL}(71.4 \mu \mathrm{M})$, whereas it decreases by a factor of 100 upon the addition of $\mathrm{Mg}^{2+}$ and $\mathrm{K}^{+}$. Increasing the temperature further decreases $c_{c}$. Actin filaments are not symmetric concerning both ends. In principle, actin monomers are added to and removed from both ends of the filaments. However, $k_{o n}$ and $k_{\text {off }}$ are not equal for both ends, namely polymerization occurs at a slower rate $k_{o n}^{-}$for the so-called "minus-" or "pointed end" of the filament owing to a necessary conformation change whereas it is 5 - 10 times as fast $\left(k_{o n}^{+}\right)$for the "plus-" or "barbed end". Due to the fact that the rate constants differ for both ends of the filaments, we also have to deal with different critical concentrations,

$$
c_{c}^{-}>c_{c}^{+} \text {. }
$$

It has been shown that at physiological salt concentration and room temperature $c_{c}^{-}=0.5 \mu \mathrm{M}$ and $c_{c}^{+}=0.12 \mu \mathrm{M}$. [78] At a concentration of $0.16 \mu \mathrm{M}$ the filaments 
lengthen at the plus-end at the same rate as they shorten at the minus-end. The monomers "move" along the filament from the plus-end to the minus-end. This process is known as "treadmilling". [3,4]

\subsubsection{In Vitro Actin Polymerization}

Lyophilized rhodamine labeled G-actin from rabbit muscle (purchased from Cytoskeleton, Denver, USA; purity $>99 \%$ ) is stored at $-70{ }^{\circ} \mathrm{C}$. The powder is dissolved in ultrapure water (produced by a Milli-Q facility, Millipore, Schwalbach, Germany) to $10 \mathrm{mg} / \mathrm{mL}$ (in $5 \mathrm{mM}$ tris(hydroxymethyl)aminomethane-hydrochloride (Tris- $\mathrm{HCl}, \mathrm{pH} 8.0$ ), $0.2 \mathrm{mM} \mathrm{NaATP}, 0.2 \mathrm{mM} \mathrm{CaCl}_{2}, 5 \%$ sucrose, and $1 \%$ dextran). The G-actin solution is diluted in A-buffer ( $5 \mathrm{mM}$ Tris-HCl (pH 8.0), $0.2 \mathrm{mM}$ NaATP, $0.2 \mathrm{mM} \mathrm{CaCl}_{2}, 0.5 \mathrm{mM}$ DTT (dithiothreitol), and $0.002 \%$ chlorhexidine) to $0.2 \mathrm{mg} / \mathrm{mL}(4.8 \mu \mathrm{M})$ and placed on ice for two hours to allow for depolymerization of existing oligomers. This step reduces the number of polymerization nuclei and thus leads to fewer but longer filaments. The concentration of $0.2 \mathrm{mg} / \mathrm{mL}$ is also optimized for rather long, individual filaments. A higher concentrated solution will lead to more, but shorter filaments. Polymerization buffer (100 mM Tris- $\mathrm{HCl}$ (pH 7.5), $20 \mathrm{mM} \mathrm{MgCl} 2,500 \mathrm{mM} \mathrm{KCl}$, and $10 \mathrm{mM} \mathrm{ATP}$ ) is added to decrease $c_{c}$ to $<0.03 \mathrm{mg} / \mathrm{mL}$ and to initiate the polymerization process, and the solution is incubated at room temperature for two hours (volume ratio A-buffer : polymerization buffer $=9: 1$ ). The solution is diluted to a final monomer concentration of $70 \mathrm{nM}$ in stabilization buffer to prevent actin filaments from depolymerizing (A-buffer, polymerization buffer (9:1), and $70 \mathrm{nM}$ phalloidin). The solution is thoroughly mixed and kept at $4{ }^{\circ} \mathrm{C}$ until it is used for the experiments. Polymerized actin can be kept at $4{ }^{\circ} \mathrm{C}$ for several weeks without observable degradation of the filaments.

Stabilization with phalloidin increases the persistence length of the filaments by about a factor of two, [26] a fact which we have to keep in mind when transferring any results to an in vivo system. However, the filaments remain semiflexible and the general results are thus still valid. It is important to stabilize filaments and dilute the solution in one and the same experimental step. If the filaments are first stabilized, they form rather dense networks which cannot be diluted easily afterwards. We found that the method of stabilizing fluorescently labeled actin with unlabeled phalloidin is superior to using labeled phalloidin with unlabeled actin since the background fluorescence is distinctively reduced. We have chosen TRITC (tetramethylrhodamine-isothiocyanate) as fluorescent dye for the system. The maximum of the absorption spectrum is at a wavelength $\lambda=554 \mathrm{~nm}$, the maximum of 
the emission spectrum at $\lambda=573 \mathrm{~nm}$. To avoid photobleaching and breakage of the filaments during observation and exposure to light, antifade solution $(77 \mathrm{mM}$ glucose, $11.2 \mathrm{mM}$ DTT, $183 \mathrm{U} / \mathrm{mL}$ glucose oxidase, $0.2 \mathrm{mM} \mathrm{NaATP}, 5 \mathrm{mM}$ Tris$\mathrm{HCl}$ ( $\mathrm{pH} 8.0$ ), $0.2 \mathrm{mM} \mathrm{CaCl}_{2}$, and $0.06 \mathrm{mg} / \mathrm{mL}$ catalase) is added to the actin solution just before filling the microchannel chamber (volume ratio actin solution : antifade $=5: 1$ ). Polymerization buffer and ATP are from Cytoskeleton, the enzymes from Roche Diagnostics (Indianapolis, USA), all other chemicals from Sigma (Taufkirchen, Germany).

\subsubsection{Collagen Characterization}

A $10 \mathrm{mg} / \mathrm{mL}$ solution of collagen I (from calf skin, USB Corporation, Cleveland, $\mathrm{OH}, \mathrm{USA})$ in $0.075 \mathrm{M}$ acetic acid $(\mathrm{AcH}, \sim \mathrm{pH} 3)$ is used. The $\mathrm{pH}$ of the $\mathrm{AcH}$ solution is slightly increased to 3.7 once collagen is dissolved in it. However, we will disregard this buffering effect here in order to simplify the description of the system. Assumedly, the difference in the absolute value of the $\mathrm{pH}$ only weakly affects the $\mathrm{pH}$ conditions discussed here. The collagen solution obtained from the manufacturer contains a combination of alpha subunits $\left(\alpha_{1}, \alpha_{2}\right)$, dimers $\left(\beta_{11}, \beta_{12}\right)$ and trimers $\left(\gamma_{1}, \gamma_{2}\right)$ as determined by SDS polyacrylamide gel electrophoresis (SDS-page, see figure 3.2). We assume that the $\gamma$ trimers $\left(M_{W} \sim 285 \mathrm{kDa}\right)$ represent triple helical

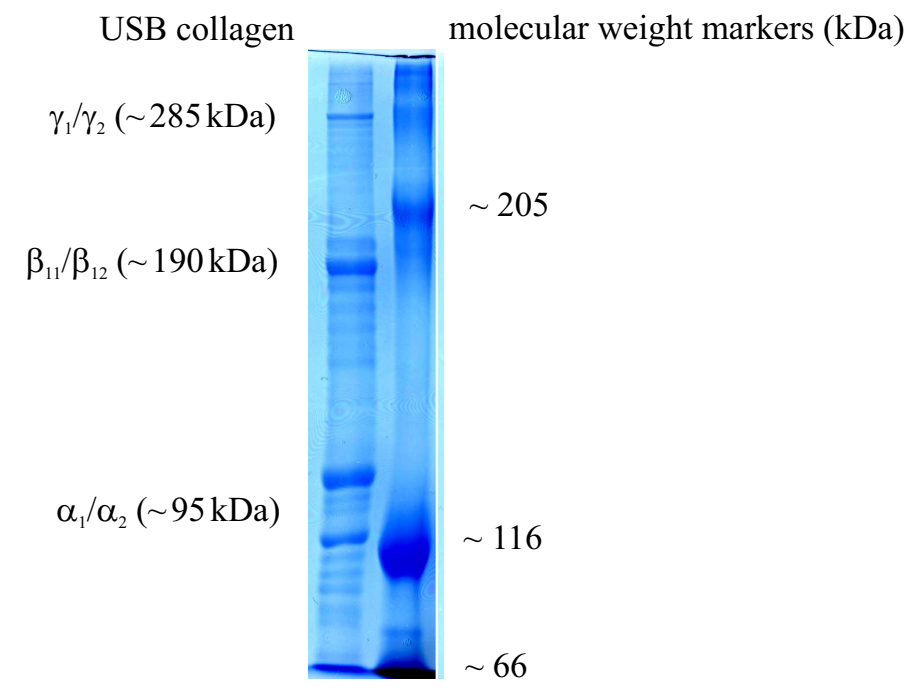

Figure 3.2: SDS page of the used collagen solution containing a combination of alpha subunits, dimers and trimers.

collagen molecules which are stable at acidic $\mathrm{pH}$ and assemble into fibrils at neutral to basic $\mathrm{pH}$. 


\subsection{Microfluidic Devices}

\subsubsection{Soft Lithography}

Just as silicon revolutionized the microelectronics industry in the 1980's, new elastomeric materials progress the development of micrometer scale fluidic devices now, 20 years later.[79] Even though silicon as well as glass are preferable materials whenever electronic or mechanical properties are desired, most applications in chemistry and biology require different attributes. For example, optical transparency and chemical inertness are much more salient. The transparent elastomer PDMS (poly(dimethysiloxane), $\left.\mathrm{CH}_{3}\left(\mathrm{SiO}\left(\mathrm{CH}_{3}\right)_{2}\right)_{n} \mathrm{Si}\left(\mathrm{CH}_{3}\right)_{3}\right)$ provides a very good alternative to microstructured silicon. Advantages include that it is flexible and thus conforms to other surfaces, chemically inert, and surfaces can easily be functionalized. PDMS is mixed with a crosslinker, poured onto the master structure, cured, and peeled off the master for use in experiments. Already in 1998 a method called "rapid prototyping" has been introduced [80] which in principle allows for the experimental realization of new ideas within 24 hours. The desired structures are drawn with a CAD program and printed on a transparency using a high resolution printer. The transparency is used as a lithography mask to fabricate the master structures by means of standard photolithography. A significant advantage of this technique is that one silicon master can be used repeatedly to fabricate replicas, which ensures the cost and time effectiveness of the method.

To fabricate the master structures of the microchannels standard photolithography techniques are used.[81,82] In figure $3.3 \mathrm{a}-\mathrm{d}$ the principle steps of this technique are

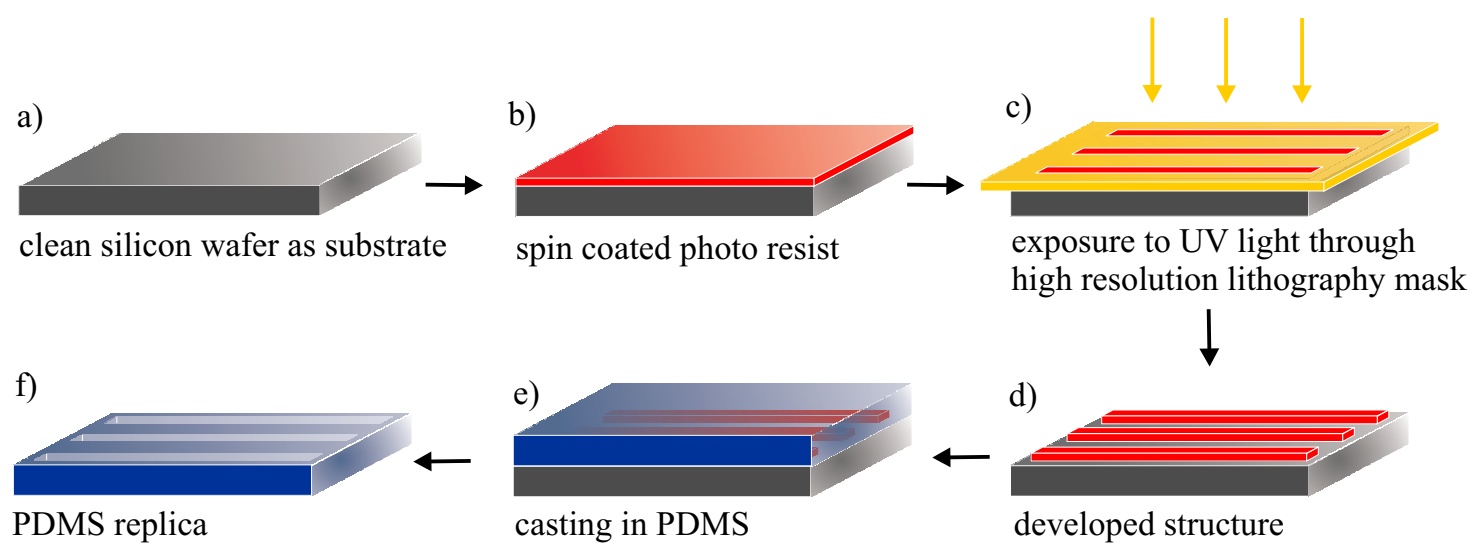

Figure 3.3: Schematic representation of the principle steps in soft lithography. a - d) Conventional photolithography with negative resist. e - f) Casting the microstructures in PDMS. 
shown. Silicon wafers are cleaned with isopropanol and dried with nitrogen and on a hotplate $\left(5 \mathrm{~min}\right.$ at $\left.200{ }^{\circ} \mathrm{C}\right)$. Depending on the experimental requirements, two different formulations of SU-8 negative photo resist (Micro Resist Technology GmbH, Berlin, Germany) are used, namely SU-8 2 and SU-8 50. The less viscous SU-8 2 resist requires a thin layer of Omnicoat (Micro Resist Technology GmbH) to promote adhesion of the photo resist to the silicon wafer (for this step, the spin speed is ramped to $500 \mathrm{rpm}$ in $5 \mathrm{~s}$, then to $2000 \mathrm{rpm}$ in $7 \mathrm{~s}$, and held for $30 \mathrm{~s}$ at $2000 \mathrm{rpm}$; then the sample is baked for $1 \mathrm{~min}$ at $200{ }^{\circ} \mathrm{C}$ ). The wafers are spin coated with a thin layer $(0.9-35 \mu \mathrm{m})$ of SU-8 negative photo resist. The photo resist is then selectively exposed to UV light at a wavelength $\lambda=365 \mathrm{~nm}$ using a Karl Süss MJB3 mask aligner (Süss Microtech AG, Garching, Germany). For resist layers thicker than a few micrometers the exposure has to be divided into several steps, allowing the resist to relax in-between for about $1 \mathrm{~min}$. A high resolution chrome mask or a lithography transparency is used as pattern, depending on the desired lateral resolution ( $\sim 1 \mu \mathrm{m}$ or $\sim 10-100 \mu \mathrm{m}$, respectively). The microstructures are drawn using AutoCAD 2005 (Autodesk, München, Germany). Lithography transparencies are ordered from JD-Photo-Tools Ltd. (Oldham, UK) and the chrome masks from ML\&C (Jena-Maue, Germany). To completely crosslink the exposed areas of the photo resist the wafers are post exposure baked and developed. In table 3.1 an overview of the parameters which are used is given.

\begin{tabular}{|l||l|l|l|}
\hline resist/thickness & SU-8 $50 / 35 \mu \mathrm{m}$ & SU-8 $2 / 1.4 \mu \mathrm{m}$ & $\mathrm{SU}-82 / 0.9 \mu \mathrm{m}$ \\
\hline \hline spin coating & $10 \mathrm{~s} \mathrm{@} 700 \mathrm{rpm}$ & $\begin{array}{l}\text { in } 5 \mathrm{~s} \text { to } 500 \mathrm{rpm} \\
\text { in } 7 \mathrm{~s} \text { to } 2000 \mathrm{rpm} \\
\end{array}$ & $\begin{array}{l}\text { in } 5 \mathrm{~s} \text { to } 500 \mathrm{rpm} \\
\text { in } 17 \mathrm{~s} \text { to } 5000 \mathrm{rpm}\end{array}$ \\
& $45 \mathrm{~s} @ 3000 \mathrm{rpm} @ 2000 \mathrm{rpm}$ & $30 \mathrm{~s} @ 5000 \mathrm{rpm}$ \\
\hline soft bake & $45 \mathrm{~min} @ 90^{\circ} \mathrm{C}$ & $\begin{array}{l}1 \mathrm{~min} @ 65^{\circ} \mathrm{C} \\
3 \mathrm{~min} @ 95^{\circ} \mathrm{C}\end{array}$ & $\begin{array}{l}1 \mathrm{~min} @ 65{ }^{\circ} \mathrm{C} \\
3 \mathrm{~min} @ 95{ }^{\circ} \mathrm{C}\end{array}$ \\
\hline exposure & & $150 \mathrm{~mJ}$ & $75 \mathrm{~mJ}$ \\
\hline post exposure bake & $10 \mathrm{~min} @ 95^{\circ} \mathrm{C}$ & $\begin{array}{l}1 \mathrm{~min} @ 65^{\circ} \mathrm{C} \\
6 \mathrm{~min} @ 95\end{array}$ & $\begin{array}{l}1 \mathrm{~min} @ 65{ }^{\circ} \mathrm{C} \\
6 \mathrm{~min} @ 95\end{array}$ \\
\hline
\end{tabular}

Table 3.1: Parameters used for photolithography.

The wafers with the developed structures as shown in figure $3.3 \mathrm{~d}$ are treated with heptafluoropropyl-trimethylsilane (97\%, Sigma) to ensure that the surface is hydrophobic and the cured PDMS will easily come off the silicon wafer and resist structure. We place the samples together with a small amount of silane (about $10 \mu \mathrm{L}$ per two-inch wafer) in a desiccator, evacuate the system and let the silane adsorb from the gas phase to the surfaces during 2 hours. Sylgard 184 PDMS (Dow Corning GmbH, Wiesbaden, Germany) is mixed with the crosslinker (ratio $10: 1$ ), 
degassed thoroughly, poured onto the three-dimensional microstructures, and cured for 4 hours at $65{ }^{\circ} \mathrm{C}$. The PDMS replicas are peeled off the wafer and used for the experiments (figure $3.3 \mathrm{e}-\mathrm{f}$ ). The width $d$ of the channels is measured by optical brightfield microscopy (accuracy $\pm 200 \mathrm{~nm}$ ), their depth $h$ by confocal reflection microscopy (accuracy $\pm 100 \mathrm{~nm})$.

\subsubsection{Assembly of Microfluidic Flow Chambers}

\section{Single Molecules Experiments}

After peeling off the PDMS microstructures from the silicon wafers, holes are punched into the filling areas with a blunt needle. To obtain closed channel systems, the PDMS microstructures as well as isopropanol-cleaned glass cover slips are oxidized using a plasma cleaner (Harrick Scientific Corporation, Ossining, USA) for $10 \mathrm{~s}$ at 2 mbar and brought into contact. Immediately, a covalent bond forms which resists a static pressure up to 2 bar.

A schematic representation of the flow chambers that are developed to investigate the thermal fluctuations of actin filaments under geometric confinement (see chapter 5) is shown in figure 3.4. They consist of channels with varying widths and different geometries, and a filling area at each front end of the channels. In figure 3.4 a - b schematic representations of a flow chamber with many parallel straight channels with different widths are shown. Other configurations are possible, for example curved channels or wedges (figure 3.4c). The experiments are performed without externally applied flow. To be able to fill the dilute actin solution into the flow chambers, polyethylene tubing with an inner diameter of $280 \mu \mathrm{m}$ and an outer diameter of $610 \mu \mathrm{m}$ (SIMS Portex, Hythe, UK) is glued into the connection holes using Loctite ${ }^{\circledR}$ 406/770 glue (Henkel Loctite, München, Germany).

An external pressure of 1.5 bar is applied to both tubes in order to fill the chamber fast. Remaining air which is captured inside the flow chamber readily diffuses through the PDMS. In this respect, it is a great advantage that PDMS is very permeable to air. On the other hand, however, PDMS is also permeable to water. To demonstrate this effect, in figure 3.5 micrographs of an empty flow chamber before and after immersion in water are shown. After about two hours (thickness of the PDMS $\sim 3 \mathrm{~mm}$ ) small water droplets begin to condens at the channel walls. This proves that water indeed diffuses through the PDMS. During an experiment, once the channels are filled with aqueous solutions the diffusion of water out of the channels $\left(D \sim 10^{-9} \mathrm{~m}^{2} / \mathrm{s}[83]\right)$ leads to an intrinsic flow within the microchannels. [84] 
a)

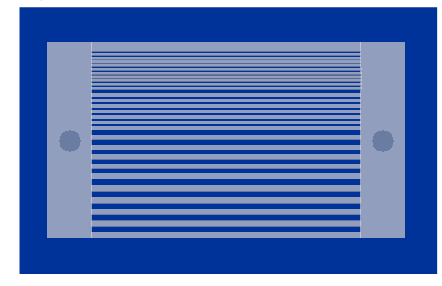

c)

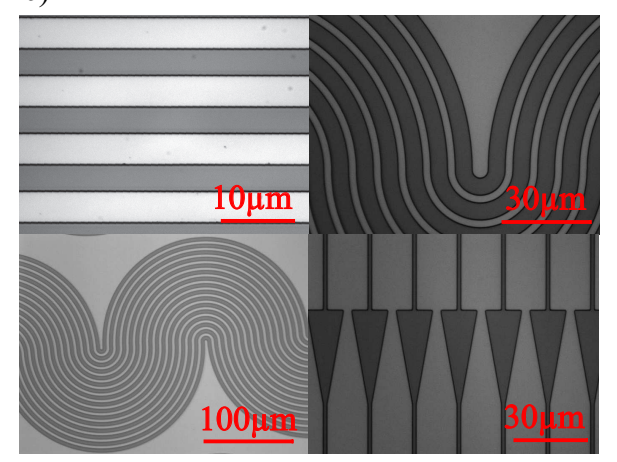

b)

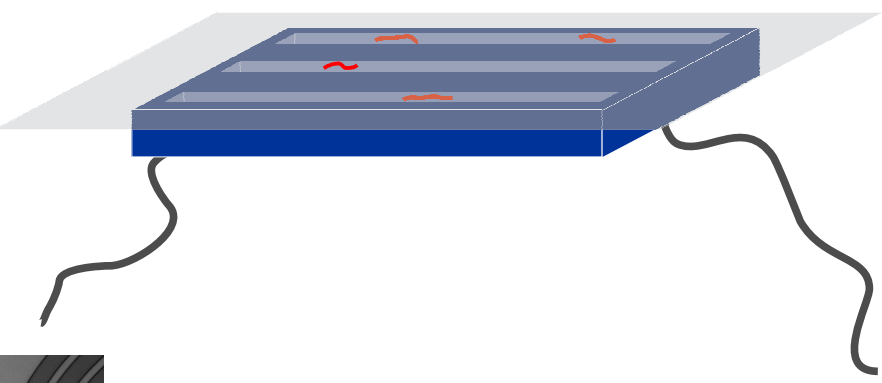

d)

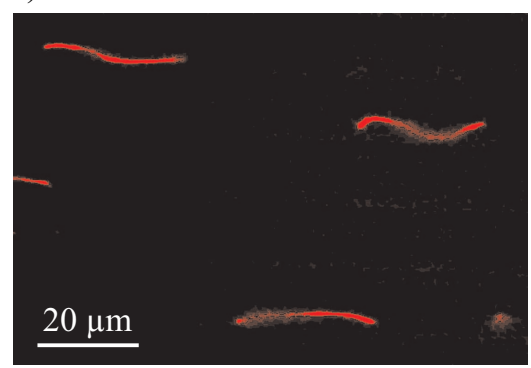

Figure 3.4: a) Schematic representation of the top view of a flow chamber with many parallel channels of different widths. Holes for the connection of the tubing are punched into both filling areas. b) Schematic representation of the assembled flow chamber. The PDMS microstructure is covered with a glass slide and tubes are glued to the connection holes. c) Micrographs of different channel geometries. d) Fluorescence micrograph of several fluctuating actin filaments in a flow chamber similar to the one sketched in a.

Since the intention of the actin experiments is to observe the fluctuations of the filaments as caused by Brownian motion, only data obtained from chambers with negligible intrinsic flow rates, which have no influence on the dynamics of the filaments, can be analyzed. To prevent flow in the microchannels we immerse the flow chamber in water to saturate the PDMS for approximately two hours before as well as during the experiment (see figure 3.6).

To avoid adhesion of biomolecules to the channel walls, the channel surfaces are coated with the protein BSA (bovine serum albumin, $1 \mathrm{mg} / \mathrm{mL}$ ) prior to performing the experiment. This process saturates the PDMS and glass surfaces. Alternatively, BSA can be added to the solution containing the biomolecules. We found that the filaments are fluctuating inside the channels without sticking for at least 24 hours. We carefully adjust our experimental system in order to enhance the reliability and reproducibility of the results. In the case of parallel straight channels the width of the microchannels $(2-10 \mu \mathrm{m})$ covers one order of magnitude, whilst their length $(2 \mathrm{~cm})$ guarantees translational invariance in the direction of the channels. F-actin naturally has a polydisperse length distribution. Since we use microfluidic chambers which include different channel widths, we are able to investigate the channel width 
a)

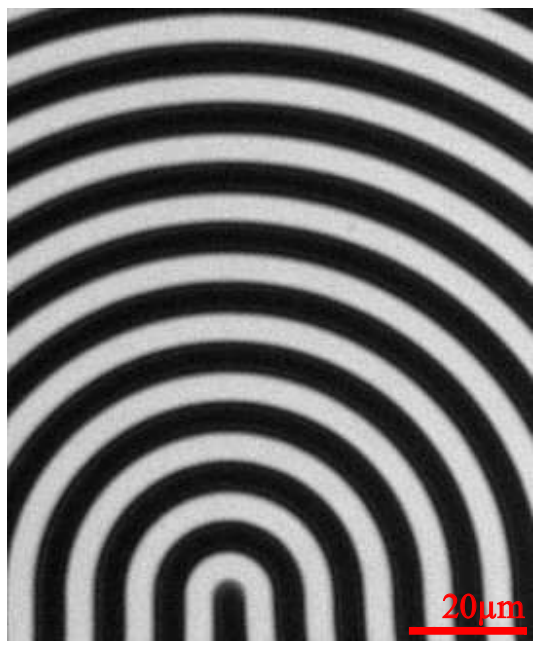

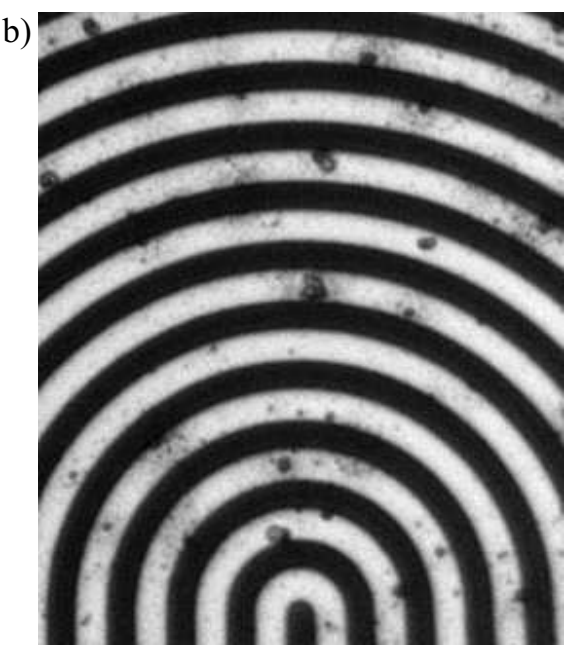

Figure 3.5: a) Flow chamber directly after assembly, the channels are empty. b) The same flow chamber after immersion in water for several hours. Small water droplets have formed at the channel walls.

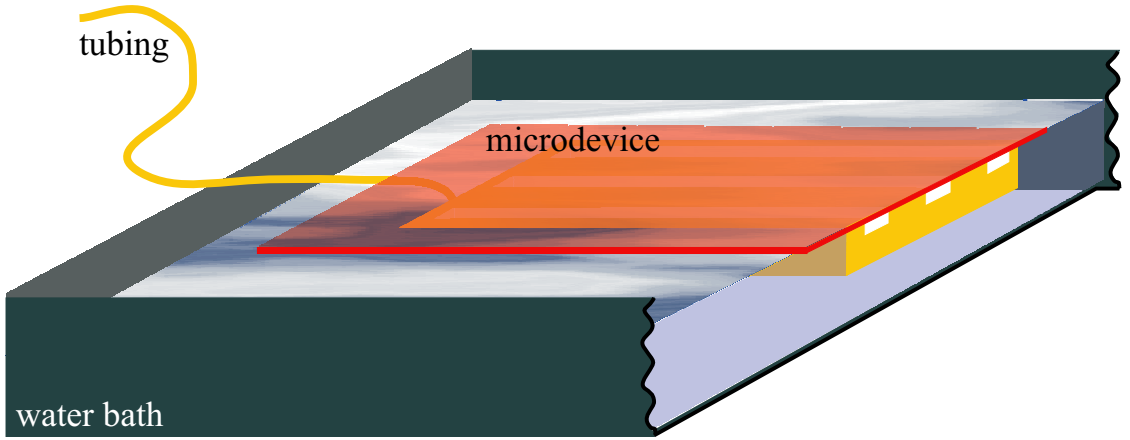

Figure 3.6: Schematic representation of a cross section of the flow chamber in a water bath. The PDMS block is completely immersed in water.

dependence and contour length dependence in a single experiment. Designing flow chambers combining many parallel channels yields the advantage that data from many individual filaments can be recorded simultaneously and additionally the flow chamber is much more reliable because single blocked channels do not affect the reminder of the system and can be disregarded. An actin concentration of $70 \mathrm{nM}$ is chosen to secure investigations of individual actin filaments in order to prevent interactions between actin filaments. We observe F-actin in a quasi two-dimensional system. This is realized by using channels with a depth of merely $h=0.9-1.4 \mu \mathrm{m}$. Furthermore, we visualize the filaments' contour in the focal plane of the microscope. 


\section{Self-Assembly of Collagen}

A further project involves the dynamic observation of collagen fibril formation and alignment (see chapter 6). To be able to apply a controlled flow and stress to the biopolymers while establishing a stable $\mathrm{pH}$ gradient, a hydrodynamical focusing device is used. [85] A schematic representation of the device is shown in figure 3.7. The device consists of two perpendicularly crossed channels $[16,17,86]$ which have

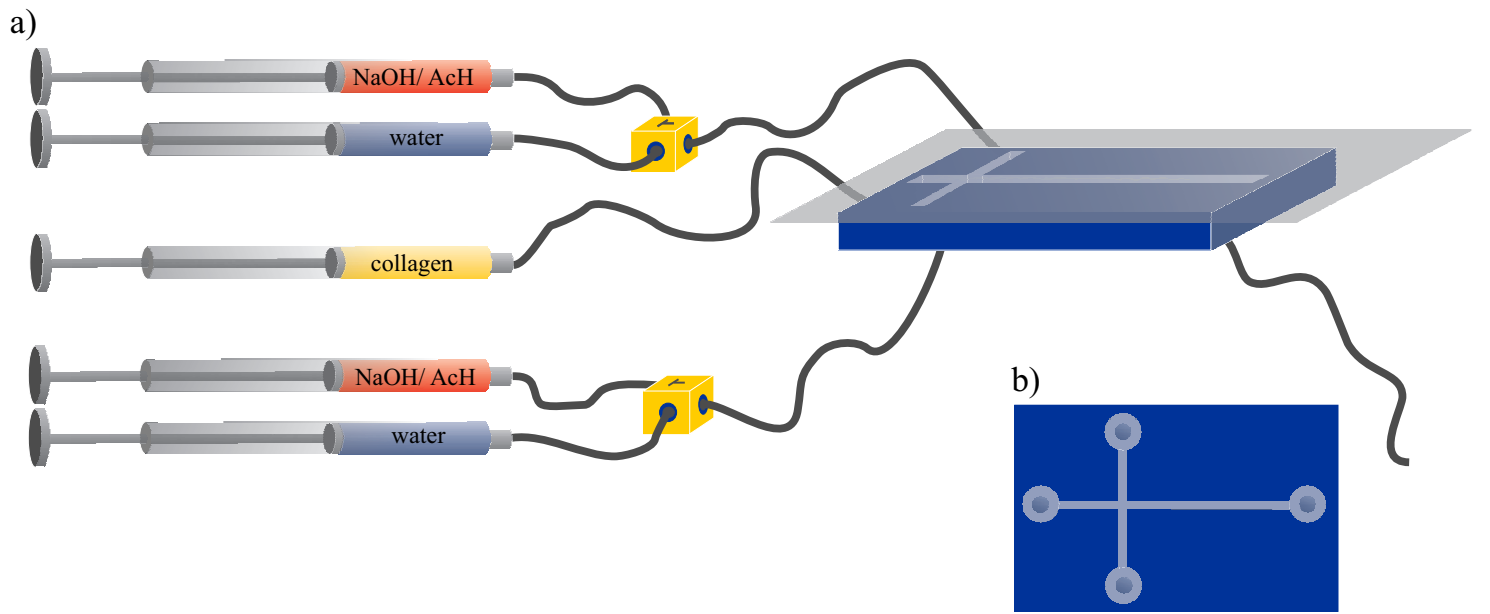

Figure 3.7: a) Setup of the microfluidic flow chamber, tubing and syringes. The main channel is connected to a syringe filled with collagen solution. The solutions in the side channels $(\mathrm{NaOH}, \mathrm{AcH})$ can be diluted with water using T-valves. b) Schematic representation of the top view of a flow chamber with crossed channel geometry. Holes are punched into all four filling areas.

a depth of $35 \mu \mathrm{m}$ and a width of $100 \mu \mathrm{m}$. The microfluidic device is connected via polyethylene (inner diameter $580 \mu \mathrm{m}$, outer diameter $965 \mu \mathrm{m}$, Becton Dickinson \& Co., Sparks, MD, USA) and Teflon ${ }^{\circledR}$ (inner diameter $500 \mu \mathrm{m}$, outer diameter $1000 \mu \mathrm{m}$, NovoDirect, Kehl, Germany) tubing to custom-made syringe pumps, which in turn are driven by programs written in LabVIEW (National Instruments Corporation, Austin, TX, USA).

Collagen solution is injected into the main channel. In order to stabilize the collagen flow before initiating the self-assembly, we first inject the solvent of the collagen solution, acetic acid solution $(\mathrm{AcH}, 0.075 \mathrm{M}, \mathrm{pH} 3)$, into the side channels. We subsequently flush the tubing and channels with ultra pure water (produced by a Millipore Milli-Q facility), and then switch the flow to sodium hydroxide $(\mathrm{NaOH})$ solution. Depending on the experimental requirements different concentrations of $\mathrm{NaOH}(0.075 \mathrm{M}-1 \mathrm{M}$, pH 13 - 14) are used. The NaOH solution can be further diluted with water using T-valves. The flow into the side channels hydrodynamically focuses the collagen stream. [85] The fluid velocity in the main channel (0.84 - 
$8.1 \mathrm{~mm} / \mathrm{s})$ is slower than the side channels $(1.26-40.5 \mathrm{~mm} / \mathrm{s})$. In this configuration, the $\mathrm{pH}$ of the collagen solution gradually increases along the length of the outlet channel resulting from diffusive mixing with the $\mathrm{NaOH} .[87,88]$ Unless otherwise stated, the chemicals are from Sigma.

\subsection{Fluorescence Microscopy}

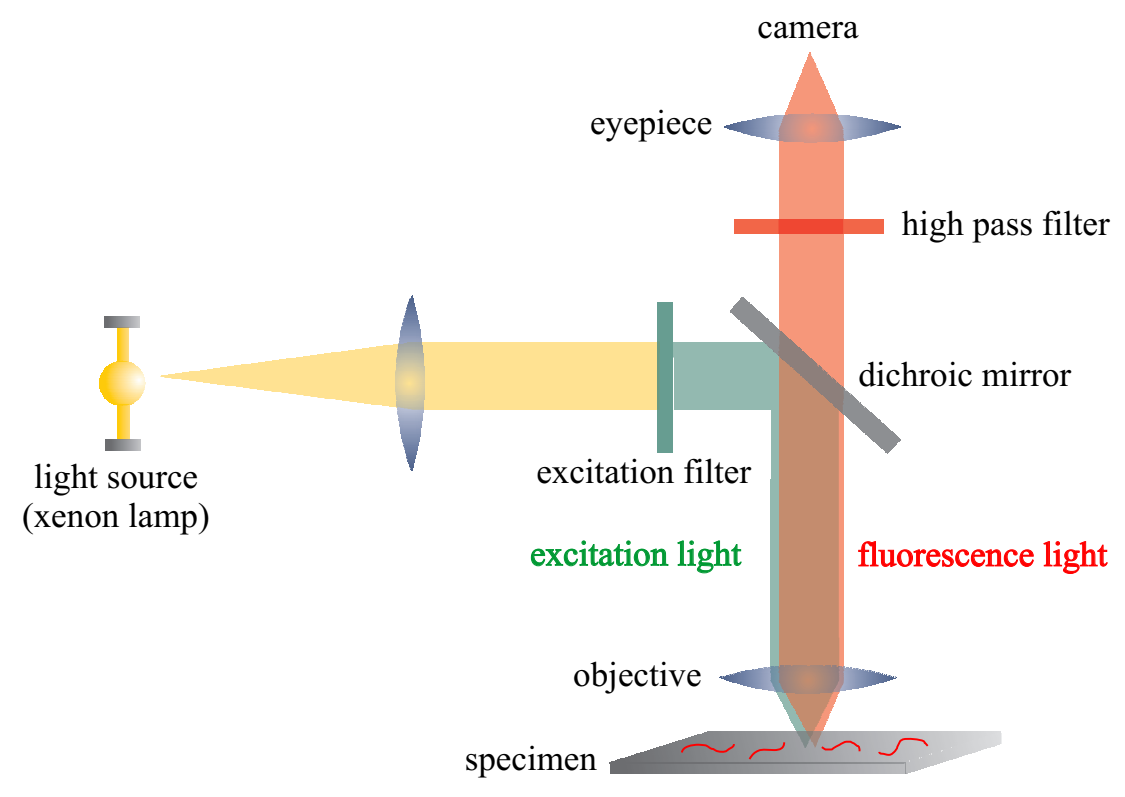

Figure 3.8: The light path for reflection fluorescence microscopy.

Fluorescence microscopy is an excellent method for our experimental purpose since actin filaments with a diameter of merely $8 \mathrm{~nm}$ cannot be visualized by conventional bright field microscopy. Using fluorescence microscopy, the diffraction disc of the dye molecules is imaged. This of course increases the visible width of the filament, but fortunately it does not strongly change the visible contour or the length of the molecule - properties in which we are predominantly interested. The light path for upright reflection fluorescence microscopy is shown in figure 3.8 . An Olympus BX61 microscope is used (Olympus Microscopy, Hamburg, Germany) equipped with a $100 \times$ Plan Apochromat oil immersion objective (N.A. 1.40). The sample is illuminated from above and the reflected light is collected in the eyepiece. The divergent light coming from the light source (in our case a $75 \mathrm{~W}$ xenon lamp, see spectrum in figure $39 \mathrm{a}$ ) is parallelized by a lens and passes the excitation filter. In accordance with the fluorescent dye (TRITC, see spectra in figure $3.9 \mathrm{c}$ ) we use a band pass with wavelengths $\lambda=530-560 \mathrm{~nm}$ (figure $3.9 \mathrm{~b}$ top). The excitation 
a)

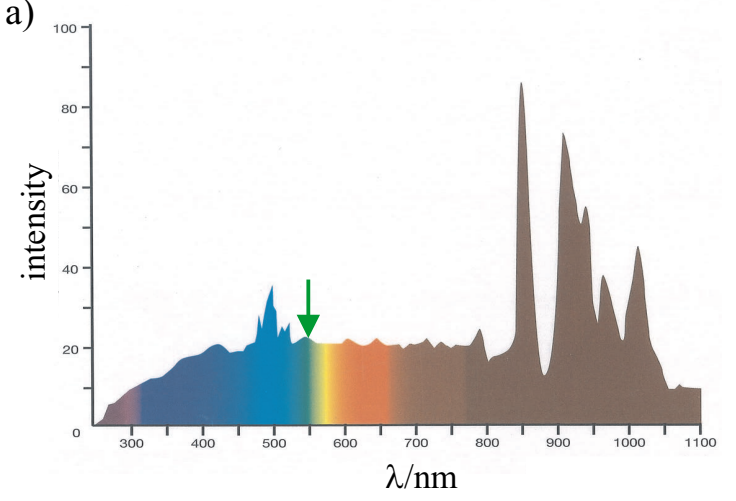

c)

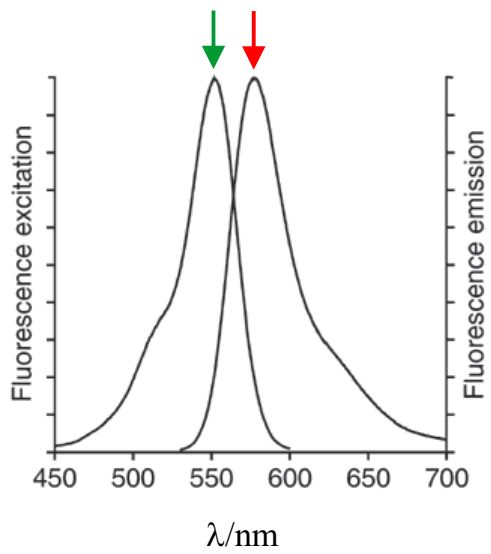

b)
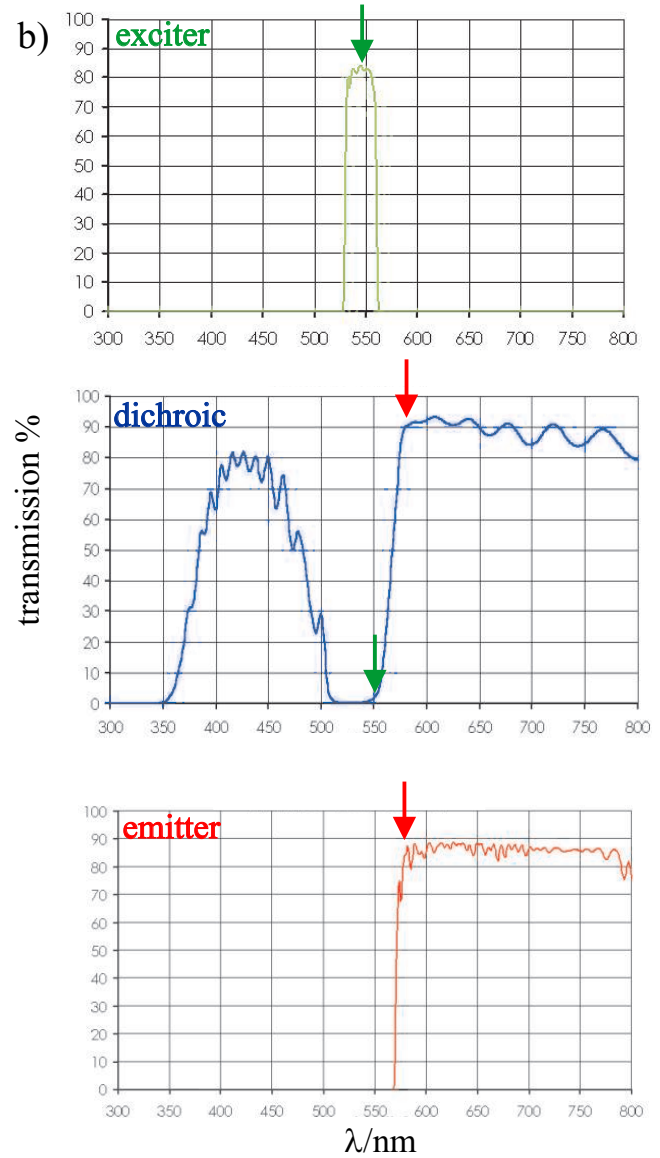

Figure 3.9: a) The emission spectrum of a xenon lamp. [89] b) The spectra of the used filters.[90] c) The absorption and emission spectra of the fluorescence dye TRITC.[91]

light with $\lambda<570 \mathrm{~nm}$ is reflected by the dichroic mirror (figure 3.9b center). The objective also serves as a condenser and focuses the light on the sample. The dye molecules absorb the (higher energy) light and emit lower energy light, which then passes the objective. The difference in wavelength is the so-called Stokes shift. The lower energy light passes the dichroic mirror (while the higher energy fraction is reflected) and finally the high pass filter which cuts off all light with wavelengths below $\lambda=572 \mathrm{~nm}$ (figure $3.9 \mathrm{p}$ bottom). All filters are purchased from AHF Analysetechnik (Tübingen, Germany). The sample can be observed through the eyepiece. Alternatively, images and movies can be recorded using a PCO SensiCamQE CCD camera (PCO, Kelheim, Germany) and the appropriate recording software Camware. For the observation of actin fluctuations the exposure time is $100 \mathrm{~ms}$ and 10 frames per second are recorded. 


\subsection{Polarized Light Microscopy}

Our microscope setup (Olympus BX61) is equipped with a polarizer and a rotatable analyzer, a $10 \times$ objective, and a halogen lamp for transmission illumination. In figure $3.10 \mathrm{a}$ the light path for polarized light transmission microscopy is shown. The divergent, non-polarized light from the light source is parallelized by a collector

a)

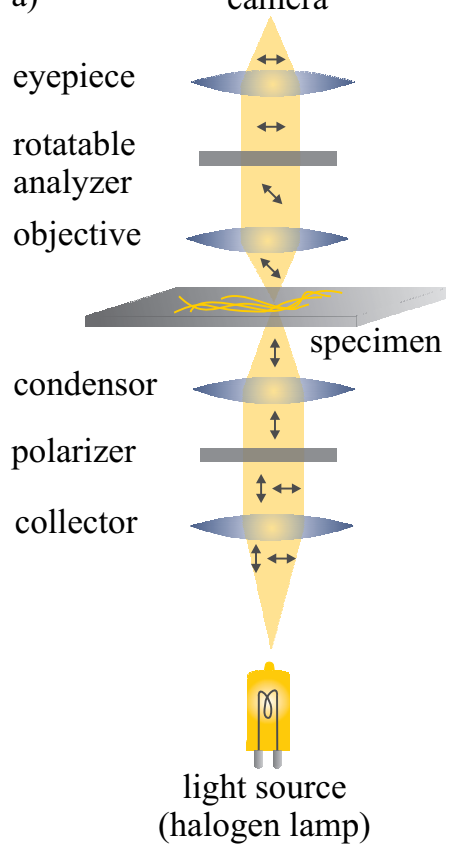

b)

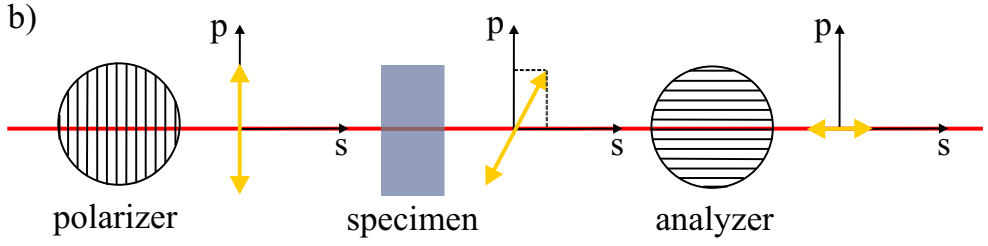

Figure 3.10: a) The light path for transmission polarized light microscopy. b) Schematic representation of the basic principle in polarized light microscopy.

lens. It subsequently passes the polarizer which leads to a parallel, polarized light beam. The light is focused onto the specimen and while passing a birefringent sample the direction of polarization is changed. An objective is used to parallelize the light beam again. The light beam reaches the eyepiece after having passed a rotatable analyzer. The direction of polarization of the analyzer is adjusted such that it is perpendicular to the (fixed) direction of polarization of the polarizer. This means that as long as there is no birefringent sample in the light path, the image is black. Only a sample which is birefringent and turns the direction of polarization of the incoming light will result in a signal.

In figure $3.10 \mathrm{~b}$ the basic principle of polarized light microscopy is shown. After passing the polarizer, one direction of polarization is blocked out of the light beam and only the p-polarized ( $\mathrm{p}$ for parallel) light remains. The analyzer in turn blocks out the p-polarized light. Thus, only the s-polarized (s for senkrecht, German: perpendicular) fraction of the light can be observed. The intensity of the observable 
signal depends on the angle by which the sample turns the direction of polarization, but in general it is lower than the original incoming intensity.

\subsection{X-Ray Microdiffraction}

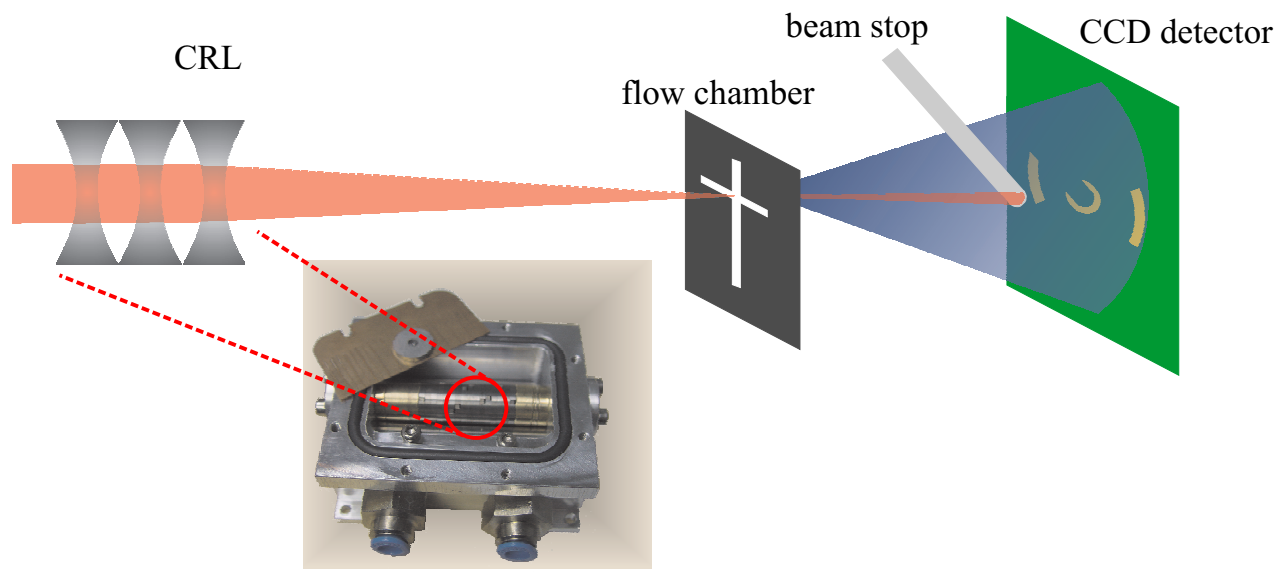

Figure 3.11: Schematic representation of the X-ray microdiffraction setup. Photograph taken from [92].

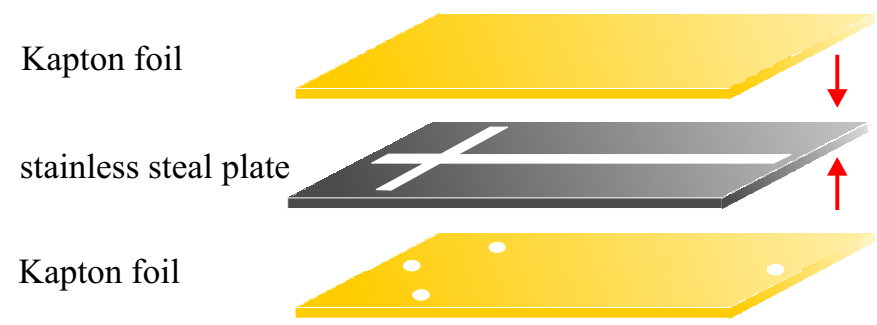

Figure 3.12: Schematic representation of the flow chamber used for X-ray experiments consisting of a cross geometry spark eroded into a stainless steel plate covered with selfadhesive Kapton ${ }^{\circledR}$ foil.

The small angle X-ray microdiffraction experiments are conducted at the beam-line ID10B of the European Synchrotron Radiation Facility (ESRF, Grenoble, France). The ID10B beam-line is a multi-purpose, high-brilliance undulator beam-line for high resolution X-ray scattering and surface diffraction on solids and liquids. [93] The microdiffraction set-up is sketched in figure 3.11. The microfluidic device is mounted onto the ID10B goniometer and the X-ray beam is adjusted by using focusing optics mounted onto a secondary stage for optical elements. Beryllium compound refractive lenses (CRL), $[94,95]$ are used to focus the X-ray beam down to a spot of $\sim 20 \mu \mathrm{m}$. The CRL have a focal distance of $\sim 1.30 \mathrm{~m}$. As a detector we use a CCD camera with fluorescent screen. The resultant 2D images of the diffraction 
patterns cover a $q$-range from 0.02 to $0.35 \AA^{-1}$. The smectic layer spacing of the liquid crystal 8CB (4'-n-octyl-4-cyanobiphenyl, Sigma) serves as calibration. The exposure time of a single image is $30 \mathrm{~s}$. A flow chamber developed specially for $\mathrm{X}$-ray experiments is used [86] as shown in figure 3.12. The cross geometry (width $150 \mu \mathrm{m}$ ) is spark eroded into a stainless steel plate (thickness $300 \mu \mathrm{m}$ ). Both sides of the steel plate are covered with self-adhesive Kapton ${ }^{\circledR}$ polyimide foil (Dr. D. Müller GmbH, Ahlhorn, Germany). Holes are punched into one of the Kapton ${ }^{\circledR}$ foils and tubing is connected to the flow chamber using homemade clips.

Additional in-house experiments are performed using a Bruker (Karlsruhe, Germany) AXS Nanostar. The setup includes a rotating anode X-ray source for $\mathrm{Cu}$ $\mathrm{K}$ radiation $(1.54 \AA)$ at a generator power of $4.05 \mathrm{~kW}$ and a virtually noise-free, real-time $2 \mathrm{D} \mathrm{Hi-Star}$ detector with photon counting ability.

\subsection{Software}

The syringe pumps are controlled by LabVIEW (National Instruments Corporation, Austin, TX, USA) programs. To record the microscopy images and movies we use Camware (PCO, Kehlheim, Germany). Image processing and data analysis is done using the commercial software AnalySIS (Soft Imaging Systems, Münster, Germany) and Image-Pro Plus (Media Cybernetics, Silver Spring, MD, USA) as well as Matlab (The Mathworks, Natick, MA, USA) programs written for this purpose. Data analysis and fit of the data curves is done using Origin (Origin LabCorporation, Northhampton, MA, USA) and C-routines written by Jan Kierfeld for this purpose. Finite element method (FEM) simulations are conducted using the commercial software Femlab (Comsol, Inc., Burlington, MA, USA). For analysis of the X-ray data we use the program fit2d by Andy Hammersley. Mathematica (Wolfram Research, Inc., Champaign, IL, USA) is used to solve systems of equations. 


\section{Chapter 4}

\section{Worm-Like Chain Model}

\subsection{Definition of the Persistence Length}

Synthetic as well as natural polymers can be classified according to their stiffness since many of their mechanical properties are in fact coupled to this characteristic. The stiffness is measured by the bending rigidity $\kappa$ of the molecule or the so-called persistence length $L_{P}$. The two measures are directly proportional:

$$
L_{P}=\frac{\kappa}{k_{B} T}
$$

where $T$ is the temperature of the system and $k_{B}$ is the Boltzmann constant. Strictly speaking we would have to mark $L_{P}$ with an index referring to the temperature for which it is valid. However, in this thesis all experiments have been conducted at room temperature and we therefore assume that $L_{P}$ generally refers to these conditions. Illustratively, $L_{P}$ is the distance along the contour of a polymer where the tangential orientation is lost; for a freely fluctuating polymer, two segments with an arc length distance $L_{P}$ behave independently.

In general, it is important to consider the ratio of $L_{P}$ and the contour length $L$ of the filament. Flexible polymers $\left(L_{P} \ll L\right)$, can be described by Gaussian statistics. [13,96-98] A biological polymer with a contour length on the order of micrometers that falls in this category is the DNA double strand $\left(L_{P}=53.4 \mathrm{~nm}\right.$ [99]). Stiff rods, where $L_{P} \gg L$, denote the other extreme. Here, a good biological example is microtubules $\left(L_{P}=5.2 \mathrm{~mm}[25]\right)$. The transition between both these ranges of stiffness is given by the so-called semiflexible polymers $\left(L_{P} \approx L\right)$, represented for example by actin filaments. 


\subsection{Unconfined Polymers}

\subsubsection{Tangent Correlation Function}
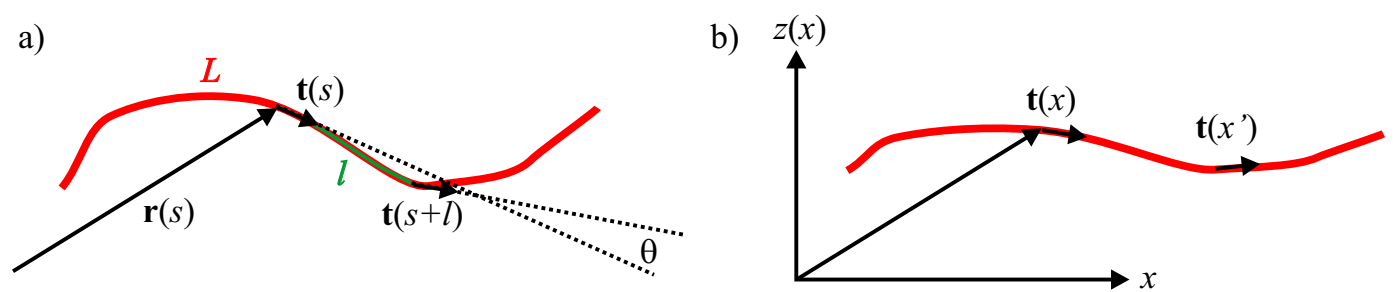

Figure 4.1: Schematic representation of a fluctuating polymer. a) Arc length parametrization, b) Monge parametrization.

Since the persistence length $L_{P}$ of semiflexible polymers is on the same order of magnitude as their contour length $L$, they cannot be described by Gaussian statistics. Instead, the worm-like chain (WLC) model $[100,101]$ is used to characterize this class of polymers. In the following section the WLC model will be discussed for two-dimensional systems, since our experimental setup physically confines the filaments to two dimensions and we observe the projection of the filaments' fluctuations merely in the focal plane of the microscope. The generalization to three dimensions will be pointed out when necessary.

The general idea of the WLC model is that the Hamiltonian describing the mechanical properties of the system only contains the bending free energy of the chain-like molecule:

$$
\mathcal{H}_{f}[\mathbf{r}(l)]=\frac{\kappa}{2} \int_{0}^{L} d l\left[\frac{\partial^{2} \mathbf{r}(l)}{\partial l^{2}}\right]^{2},
$$

were $\mathbf{r}(l)$ is the position vector on the contour of the filament parameterized by the arc length $l$ of the polymer (see figure 4.1a). The index $f$ in equation 4.2 refers to the unconfined (free) case, in contrast to equations describing spatially confined polymers that will be indexed with $c$ in the following discussion. The correlation of the unit tangent vectors $\mathbf{t}(s)=\frac{\partial \mathbf{r}(s)}{\partial s}$ can be calculated in a straightforward manner by applying a Boltzmann distribution to the energies in the system, and shows a first-order exponential decay with $L_{P}$ as a characteristic length scale:[101,102]

$$
\langle\cos \theta(l)\rangle_{f} \equiv\langle\overline{\mathbf{t}(s) \cdot \mathbf{t}(s+l)}\rangle=\exp \left(-l / 2 L_{P}\right),
$$

where $\theta$ is the angle between the two considered tangent vectors. The bar in equation 4.3 denotes the spatial averaging within one snapshot of a conformation of a filament, whereas the brackets denote the thermal (that is, time) averaging which includes 
many conformations of a filament. The two-dimensionality leads to the factor 2 in the denominator of the exponent. According to the definition that we use here $L_{P}$ is an intrinsic property of the filament. This implies that the value of $L_{P}$ is the same in two and in three dimensions and the factor 2 appears in the tangent correlation function. ${ }^{1}$

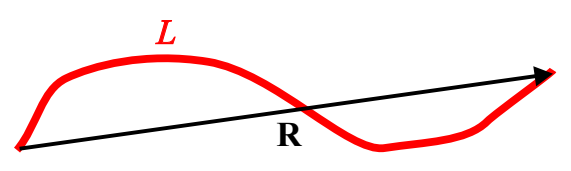

Figure 4.2: Definition of the end-to-end vector $\mathbf{R}$ of a fluctuating polymer.

The mean square end-to-end distance $\left\langle\mathbf{R}^{2}\right\rangle$ can be calculated by integrating the tangent correlation:

$$
\left\langle\mathbf{R}^{2}\right\rangle=\left\langle\int_{0}^{L} \int_{0}^{L} d l_{1} d l_{2} \mathbf{t}\left(l_{1}\right) \cdot \mathbf{t}\left(l_{2}\right)\right\rangle .
$$

Since averaging operation and integral commute we arrive at

$$
\left\langle\mathbf{R}^{2}\right\rangle=8 L_{P}^{2}\left(\frac{L}{2 L_{P}}-1+\exp \left(-\frac{L}{2 L_{P}}\right)\right)
$$

for the two-dimensional case

\subsubsection{Radial Distribution Function}

To demonstrate the principle difference between Gaussian and semiflexible chain molecules, it is instructive to compare the respective radial distribution functions. For Gaussian chains it can for many purposes be well approximated by a normal probability curve. For semiflexible polymers the description is more complex. Nevertheless, for a given $L$ and $L_{P}$ the approximate radial distribution function $G_{f}(R / L)$ for free semiflexible polymers can be calculated analytically. [35] However, one has to introduce the Monge parametrization, which is also very convenient if the fluctuations of a semiflexible polymer are to be related to some kind of external potential (see section 4.3). Only weak bends are assumed and configurations where the filament performs overhangs are neglected. The contour line can be described by a function $z(x)$ (see figure 4.1b). Since we study a two-dimensional system, $z(x)$

\footnotetext{
${ }^{1}$ Alternatively, a more mathematical definition of $L_{P}$ as the characteristic decay of the tangent correlation can be chosen. In this case, $L_{P_{2 D}}=2 L_{P_{3 D}}$ and the tangent correlation function is universal for two or three dimensions.[103]
} 
is a scalar; however, in the more general case of a three-dimensional system it is a two-dimensional vector. The assumption that the filament is only weakly bent also implies that $z^{\prime}(x) \ll 1$. In this case the second derivative of $z(x)$ equals the curvature. With these assumptions the Hamiltonian in equation 4.2 becomes

$$
\mathcal{H}_{f}[z(x)]=\frac{\kappa}{2} \int_{0}^{L_{x}} d x\left[\frac{\partial^{2} z(x)}{\partial x^{2}}\right]^{2},
$$

where $L_{x}$ is the contour length projected on the $x$-axis. For small fluctuations we can set $L_{x}=L$.

To calculate the radial distribution function the expression

$$
G_{f}(R / L)=\delta(R / L-|\mathbf{R} / L|)
$$

where $\mathbf{R}$ is the end-to-end vector (see figure 4.2 ) and $\delta(x)$ is the Dirac function, has to be evaluated. We make the rotation invariance explicit by using the normalized norm of the end-to-end vector $R / L$ as the argument of the function. In principle the end-to-end vectors of the different conformations do not necessarily have to be considered, but the radial distribution function can be analyzed for any vectors $\widetilde{\mathbf{R}}$ connecting points on the contour of the filament with an arc length distance $\widetilde{L}$. However, in section 5.3 we will analyze our data in terms of the radial distribution function of the end-to-end vectors. Therefore we consider this special case. Equation 4.7 can be solved by applying Monge parametrization and harmonic or Gaussian approximation, which implies that any orders higher than second are neglected. For the two-dimensional case this leads to [35]

$$
G_{f}(R / L)=\frac{2 L_{P}}{L \pi \mathcal{N}} \sum_{k=1}^{\infty} \pi^{2} k^{2}(-1)^{k+1} \exp \left(-\frac{2 L_{P}}{L} \pi^{2} k^{2}(1-R / L)\right),
$$

where $\mathcal{N}$ is a normalization constant. Analyzing the radial distribution function in addition to the tangent correlation function provides a second method to obtain the persistence length from the experimental data and thus verifies the consistency of the experiment and the data analysis. Combining both statistical measures, the tangent correlation and the radial distribution function, helps to complete the characterization of semiflexible polymers.

\subsection{Confined Polymers}

\subsubsection{Tangent Correlation Function}

The WLC model is based on the mere mechanics of the actin filaments. To be able to describe confined filaments an additional energy term describing the microchannels 
has to be accounted for and included into a modified WLC model. Ideally, this should be a hard wall potential implying rectangular channel walls. However, in a first approximation a parabolic wall potential $K / 2 \cdot z(x)^{2}$, where the $z$-axis is perpendicular to the direction of the channels and $K$ is a constant value determining the strength of the potential, will be discussed.[38, 104] We will show that even this very rough approximation is surprisingly well suited to describe our experimental results. Again, since we discuss a two-dimensional system $z(x)$ is a scalar, although in the more general case of a three-dimensional system we would have to consider a two-dimensional vector. The Hamiltonian using Monge parametrization is now given by

$$
\mathcal{H}_{c}[z(x)]=\int_{0}^{L_{x}} d x\left[\frac{\kappa}{2}\left(\frac{\partial^{2} z(x)}{\partial x^{2}}\right)^{2}+\frac{K}{2} z(x)^{2}\right] .
$$

For F-actin in confining channels this approximation is certainly justified. Furthermore, we assume infinitely long filaments and no special boundary conditions. We use the Fourier transform

$$
z(x)=\frac{1}{2 \pi} \int d q z(q) \exp (-i q x)
$$

and take the limit $L_{x} \rightarrow \infty$ in terms of which the Hamiltonian in equation 4.9 becomes

$$
\mathcal{H}_{c}[z(q)]=\frac{1}{4 \pi} \int_{-\infty}^{\infty} d q\left(\kappa q^{4}+K\right) z(q) z^{*}(q)
$$

Equation 4.11 shows that the modes $z(q)$ decouple. There are no mixed terms $z(q) z^{*}\left(q^{\prime}\right)$ with $q^{\prime} \neq q$. From this it follows that

$$
\left\langle z(q) z\left(q^{\prime}\right)\right\rangle=2 \pi G(q) \delta\left(q-q^{\prime}\right)
$$

In order to determine $G(q)$, we recall that Boltzmann weights $\exp \left(-\mathcal{H}_{c}[z(q)] / k_{B} T\right)$ lead to a Gaussian distribution for each mode $z(q)$ if $\mathcal{H}_{c}$ is a quadratic Hamiltonian. The expected value for a quantity with Gaussian distribution is known, and thus we obtain

$$
G(q)=\frac{k_{B} T}{\kappa q^{4}+K}
$$

First of all the height-height correlations

$$
\left\langle z(x) z\left(x^{\prime}\right)\right\rangle=\frac{1}{4 \pi^{2}} \iint d q d q^{\prime}\left\langle z(q) z\left(q^{\prime}\right)\right\rangle e^{i\left(q x-q^{\prime} x^{\prime}\right)}=\frac{1}{2 \pi} \int d q \frac{k_{B} T}{\kappa q^{4}+K} e^{i q\left(x-x^{\prime}\right)}
$$

shall be calculated. To solve the integral in equation 4.14 we close the integration path in the complex plane, analyze the poles of the denominator and apply 
the residue theorem. Furthermore, we introduce a new length scale, the so-called deflection length

$$
\lambda=\sqrt{2}\left(\frac{\kappa}{K}\right)^{1 / 4}
$$

which is characterized by the competition of bending energy and confining energy. A descriptive idea of the deflection length as the distance between two consecutive

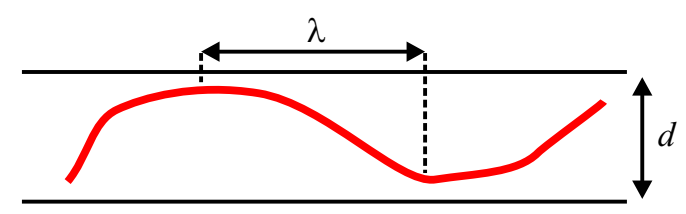

Figure 4.3: Illustration of the deflection length $\lambda$.

contact points of the filament with the channel walls and the appropriate scaling law have been introduced by Odijk.[14] He found that

$$
\lambda=a \cdot d^{2 / 3} L_{P}^{1 / 3}
$$

where $a$ is a geometry dependent coefficient and $d$ is the channel width. Equation 4.14 thus leads to

$$
\left\langle z(x) z\left(x^{\prime}\right)\right\rangle=\frac{\lambda}{4 \sqrt{2} L_{P}} \exp \left(\frac{x-x^{\prime}}{\lambda}\right) \cos \left(\frac{x-x^{\prime}}{\lambda}-\frac{\pi}{4}\right) .
$$

The angular correlations can now be calculated:

$$
\left\langle\cos \theta\left(x-x^{\prime}\right)\right\rangle \approx 1-\frac{1}{2}\left\langle\theta\left(x-x^{\prime}\right)^{2}\right\rangle,
$$

which for small fluctuations $\left(\theta \approx \tan \theta=\frac{\partial z}{\partial x}\right)$ becomes

$$
\left\langle\cos \theta\left(x-x^{\prime}\right)\right\rangle \approx 1-\frac{1}{2}\left\langle\left(\frac{\partial z(x)}{\partial x}-\frac{\partial z\left(x^{\prime}\right)}{\partial x^{\prime}}\right)^{2}\right\rangle \equiv 1-\left(g(0)-g\left(x-x^{\prime}\right)\right),
$$

where

$$
g\left(x-x^{\prime}\right)=\left\langle\frac{\partial z(x)}{\partial x} \cdot \frac{\partial z\left(x^{\prime}\right)}{\partial x^{\prime}}\right\rangle=\frac{\lambda}{2 \sqrt{2} L_{P}} \exp \left(\frac{x-x^{\prime}}{\lambda}\right) \cos \left(\frac{x-x^{\prime}}{\lambda}+\frac{\pi}{4}\right) .
$$

For small fluctuations we can now set $\left(x-x^{\prime}\right)=l$ and obtain an analytical solution for the tangent correlation function for confined filaments:

$$
\langle\cos \theta(l)\rangle_{c}=1-\frac{\lambda}{2 \sqrt{2} L_{P}}\left(\cos \left(\frac{\pi}{4}\right)-\cos \left(\frac{\pi}{4}+\frac{l}{\lambda}\right) \exp \left(\frac{-l}{\lambda}\right)\right) .
$$

In addition to $L_{P}$ which describes the mechanical rigidity of the actin filaments, the deflection length $\lambda$ describing the channel geometry appears as a fitting parameter 
in equation 4.21. Note that the scaling arguments by Odijk, as well as more rigorous calculations of the deflection length, were deduced for semiflexible filaments in cylindrical hard wall tubes of diameter $d,[14,43]$ whereas a parabolic potential is used to describe our system.

To show that the tangent correlation function in equation 4.21 for $K \rightarrow 0(\lambda \rightarrow \infty)$ approaches the free tangent correlation in equation 4.3 we expand both expressions in series around $l=0$ taking into account that all terms with $\lambda$ in the denominator approach zero for small $K$. For unconfined filaments we arrive at

$$
\langle\cos \theta(l)\rangle_{f}=1-\frac{l}{2 L_{P}}+\mathcal{O}\left(l^{2}\right)
$$

whereas for confined filaments the tangent correlation is

$$
\langle\cos \theta(l)\rangle_{c}=1-\frac{\lambda}{2 \sqrt{2} L_{P}}\left(\frac{1}{\sqrt{2}}-\left(\frac{1}{\sqrt{2}}-\frac{\sqrt{2} l}{\lambda}+\mathcal{O}\left(l^{2}\right)\right)\right) .
$$

A comparison of both terms yields

$$
\langle\cos \theta(l)\rangle_{c}=\langle\cos \theta(l)\rangle_{f}=1-\frac{l}{2 L_{P}}
$$

Equation 4.3 can therefore be considered as a special case of equation 4.21 for infinitely small potential strength $K$.

In the following we will discuss the course of the tangent correlation under the influence of a confining potential in more detail. For large arc length distances $l$, the tangent correlation function described by equation 4.21 does not decay to zero in the same manner as the tangent correlation for unconfined filaments (equation 4.3). Instead, it approaches a constant asymptotic non-zero value:

$$
\langle\cos \theta(l)\rangle_{\infty} \approx 1-\frac{\lambda}{4 L_{P}}=\text { const. } \neq 0
$$

We observe a characteristic oscillation along the tangent correlation function whose wavelength is set by the external potential. Determining the position of the first local minimum $l_{\text {min }}$ of the modified tangent correlation (equation 4.21), we find that it depends on the deflection length but not on the persistence length:

$$
\lambda=\frac{2}{\pi} l_{\text {min }}
$$

This provides a suitable method to deduct the deflection length from the experimental results. 


\subsubsection{Radial Distribution Function}

The radial distribution function is a central quantity of single polymers. In contrast to the tangent correlation function, which demands knowledge of the complete course of the contour of the filament, it can also be analyzed in the case of polymers bound in networks. As in the case of unconfined polymers (equation 4.8) the Monge parametrization and Gaussian approximation are presumed. If the confining potential is again considered to be quadratic the radial distribution function can be calculated.[36] For the two-dimensional case we obtain:

$$
G_{c}(R / L)=\frac{1}{\mathcal{N} \pi} \sum_{k=1}^{\infty} \exp [-F(k)(1-R / L)] F(k)(-1)^{k+1} \frac{\sqrt{e} \pi p_{4}(e)}{k \sin \frac{\pi \sqrt{e}}{k}} \cdot \frac{1-e / k^{4}}{1+e / k^{4}}
$$

where

$$
\begin{gathered}
F(k)=\frac{2 L^{3} L_{P}}{\pi^{2} k^{2} \lambda^{4}}+\frac{2 L_{P} \pi^{2} k^{2}}{L}, \\
e=\frac{L^{4}}{\pi^{4} \lambda^{4}}
\end{gathered}
$$

and

$$
p_{4}(e)=\prod_{j=1}^{\infty}\left(1+\frac{e}{j^{4}}\right) .
$$

Note that we set $L_{x}=L$ for these calculations which is a valid approximation for the case of small fluctuation which is considered here. For $K \rightarrow 0(\lambda \rightarrow \infty)$ we obtain once more equation 4.8, which describes the radial distribution function of unconfined filaments. In this case $F(k)$ is reduced to its second summand, $e=0$, consequently $p_{4}(e)=1$. A series expansion of $x / \sin x$ around zero shows that the respective factor in equation 4.27 approaches the value 1 .

Equation 4.27 contains three free parameters: $L, L_{P}$, and $\lambda$. The distribution becomes narrower and is shifted towards larger values of $R / L$ if the filaments are shorter (decrease of $L$ ), stiffer (increase of $L_{P}$ ), or more confined (decrease of $\lambda$ ). Since $L$ can usually be directly deduced from micrographs, the same set of free parameters, $L_{P}$ and $\lambda$, remains as in the tangent correlation function. Therefore we are able to analyze both quantities independently and cross-check the results for consistency.

\subsubsection{Segment Distribution}

A confining potential also influences the temporal and spatial distribution of segments of the filament within the conformational space. If we consider unconfined 


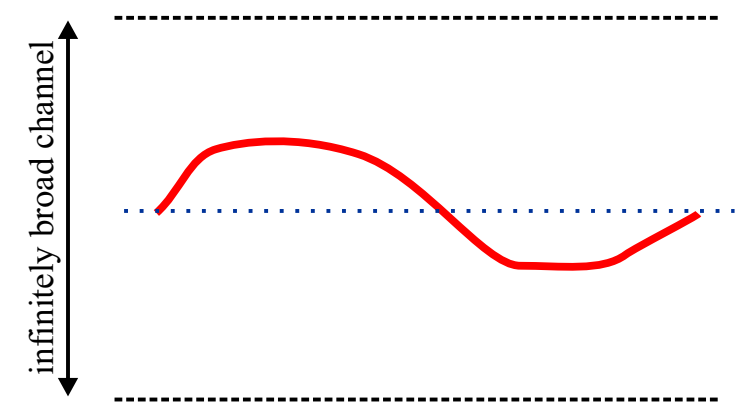

Figure 4.4: Illustration of the procedure to determine the thermal roughness exponent $\zeta$.

polymers, their spatial distribution is determined merely by the so-called thermal roughness exponent $\zeta=3 / 2$. That is to say, if we imagine a main direction (or an infinitely broad channel) in the system (dashed lines in figure 4.4) the probability to find the two ends of the polymer at the same vertical position (dotted line) scales with $L^{-\zeta}=L^{-3 / 2} \cdot[105-107]$

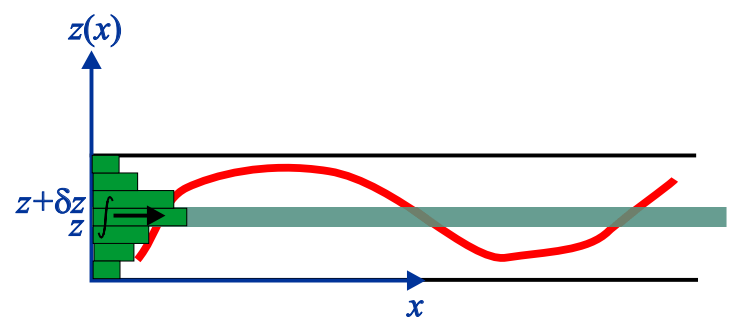

Figure 4.5: Illustration of the procedure to determine the segment distribution.

If a confining wall potential comes into play, it induces deflections of the polymers at the wall and competes with the bending rigidity of the molecules. We consider semiflexible polymers in the proximity of a surface or in the presence of two walls (that is, in a channel). The exponents related to the segment distribution perpendicular to the wall $\left(\theta_{r}, r\right.$ for "repulsion") and to the return probability to the wall along the polymer (parallel to the wall, $\chi_{r}$ ) can be obtained by calculating the probability $P(z)$ to find a segment of a filament within a range $[z, z+\delta z]$ as a function of $z$ (see figure 4.5). It can be shown that

$$
P(z) \propto\left(\frac{\lambda}{L_{P}}\right)^{-\chi_{r}}\left(\frac{z}{L_{P}}\right)^{\theta_{r}+1 / 3} .
$$

From normalization requirements it follows that $\theta_{r}$ and $\chi_{r}$ are not independent but

$$
\chi_{r}=2+\zeta \theta_{r}=2+\frac{3 \theta_{r}}{2} .
$$

It has been shown that $\theta_{r}=1 / 3$ and $\chi_{r}=5 / 2$.[105-107] Thus, the probability to find a segment of the filament at a distance $z$ close to the channel wall scales with 
$z^{2 / 3}$ and the absolute value of the probability at a certain distance $z$ is proportional to $\lambda^{-5 / 2}$. The relation 4.31 is true for the behavior of filaments in the presence of one or two walls since a segment of a polymer is influenced by only one wall. However, in the case of a confining channel we have to keep in mind that $\lambda$ plays the role of the longest free segment instead of $L$, as in the case of only one wall. Thus, in addition to the scaling relation between $\lambda, L_{P}$, and $d$ (equation 4.16), a second scaling law for semiflexible polymers is obtained. Note that these scaling laws are independent of the considered form of the confining potential and also hold in the exact case of a rectangular hard wall potential. Hence we can in some respect consider the data analysis in terms of scaling laws as a more general approach than the description of tangent correlation function and radial distribution function. 


\section{Chapter 5}

\section{F-Actin in Microfluidic Environment}

Single actin filaments shall be investigated in an environment which resembles their native surrounding: a polymer network with a mesh size on the micrometer scale. We analyze the mechanics of the biopolymers under the influence of confining microchannels. The system is characterized by the stiffness of the biopolymers and the confining potential, which we determine by analyzing the tangent correlation functions, the radial distribution functions, and the segment distributions for different channel geometries.

\subsection{Image Processing and Data Analysis}

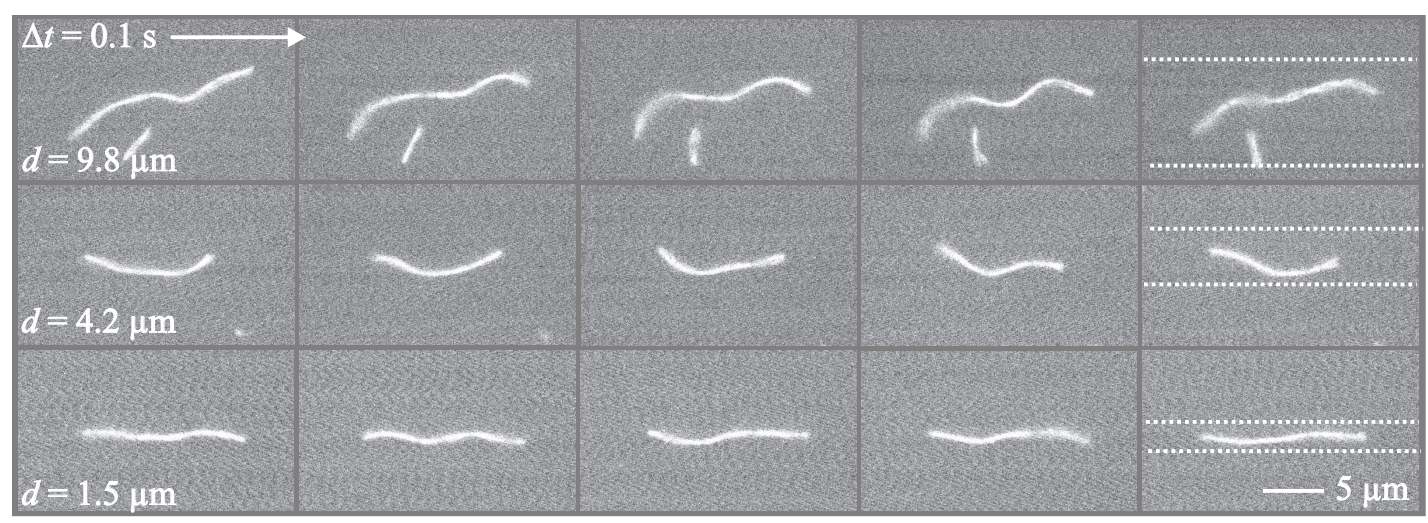

Figure 5.1: Snapshots of fluctuating actin filaments in channels of different widths. The elongation of the biopolymers increases with decreasing channel width. The white dotted lines mark the positions of the channel walls which are not visible in the fluorescence micrographs. 


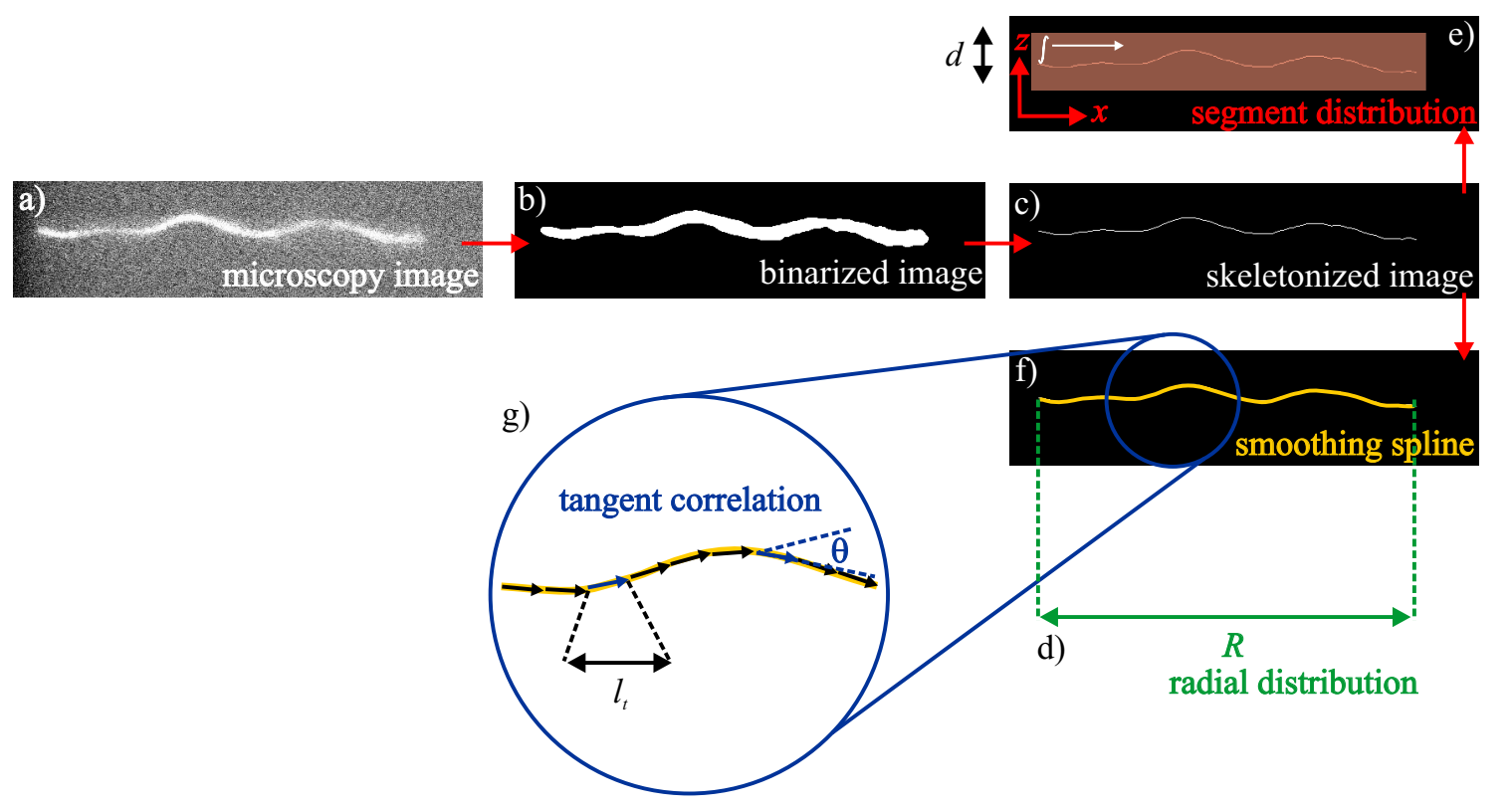

Figure 5.2: Step by step representation of image processing and data analysis. a) The statistical noise in the original micrographs is reduced and the images are b) binarized. c) Subsequently, the contour is skeletonized. d) The radial distribution function is obtained by determining the end-to-end distance of the contour. e) To obtain the segment distribution the intensity is integrated in the direction of the channels along the full length of the polymer and plotted against the $z$-coordinate over the complete channel width $d$. f) A smoothing spline fit is applied and $\mathrm{g}$ ) the tangent correlation function is determined.

Snapshots of the fluctuating actin filaments are taken and the contour is analyzed in order to determine the tangent correlation function, the radial distribution function of the end-to-end distances, and the segment distribution. In figure 5.1 three different series of such snapshots are shown. Obviously, the smaller the channel width $d$ is the more elongated the filaments are, whereas in the channels with $9.8 \mu \mathrm{m}$ they almost behave like unconfined filaments. The 16 bit grayscale images (figure $5.2 \mathrm{a}$ ) are recorded using a PCO SensiCamQE CCD camera and the appropriate recording software Camware. The image processing software AnalySIS is used to apply an $\mathrm{N} \times \mathrm{N}$ filter to the images, which reduces statistical noise by assigning the averaged gray value of a square area of the image to the central pixel of this square. The size of the square and the number of iterations are determined individually for each data set and usually lie in-between $3 \times 3-5 \times 5$ and $1-4$, respectively. A threshold is set and the images are binarized (figure $5.2 \mathrm{~b}$ ). In the following step, the images of the filaments are skeletonized to a one-pixel-line using the image processing software Image-Pro Plus (figure 5.2 c).

The radial distribution function is obtained by measuring the end-to-end distance $R$ for each image, that is to say, each conformation, of one data set (figure $5.2 \mathrm{~d}$ ). The 
contour length $L$ corresponds to the length of the one-pixel-line. To obtain the segment distribution, we rotate all images such that the channel direction is horizontal. We integrate the intensity along the full filament length along the direction of the channel, average the values over all recorded conformations of a filament, normalize the result by the length of the filament, and plot the result against the $z$-coordinate perpendicular to the channel. In the resulting graph one channel wall is positioned at $z=0$ while the second channel wall lies at $z=d$ (figure $5.2 \mathrm{e}$ ).

Analysis of the tangent correlation function is conducted using a Matlab program written for this purpose. A smoothing spline fit is applied to the one-pixel-line (figure $5.2 \mathrm{f}$ ). An arc length reparametrization of the fitted line is obtained by dividing it into tangent vectors of equal length (figure $5.2 \mathrm{~g}$ ). The correlation of these tangent vectors as a function of the arc length $l$ is given by their scalar product. Although

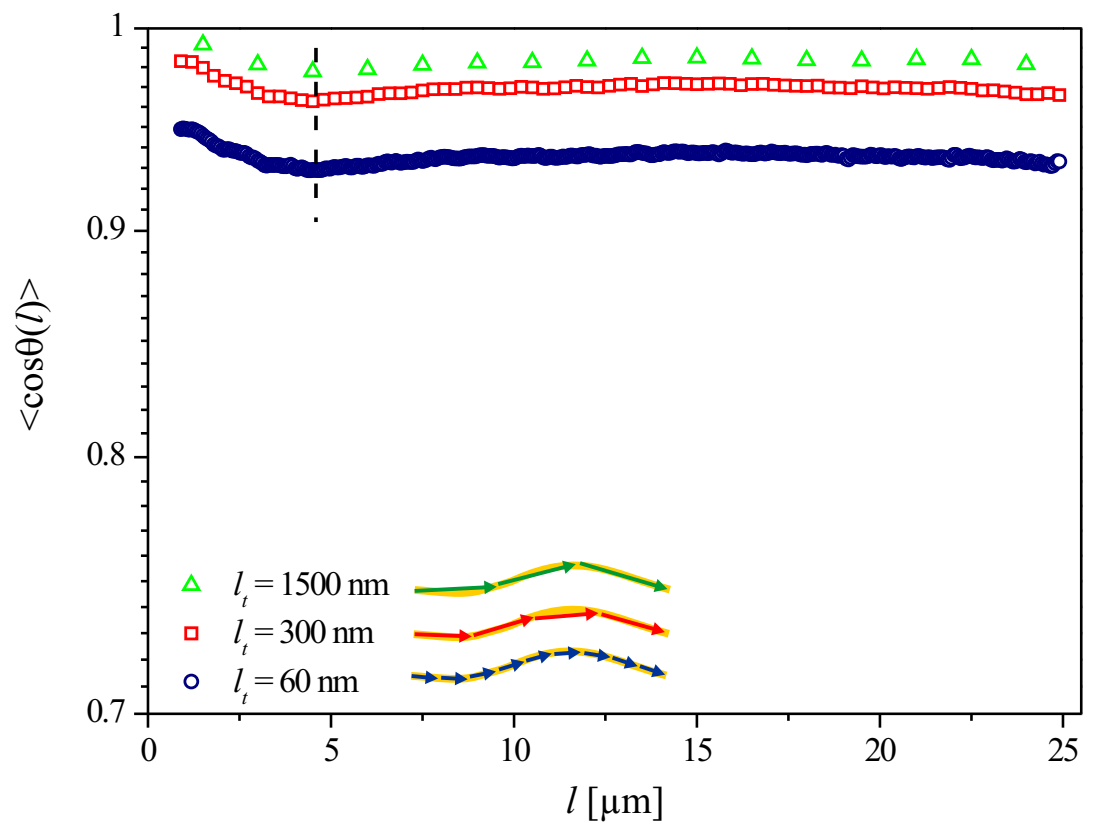

Figure 5.3: Data set analyzed by choosing different values for $l_{t}$. The vertical position but not the horizontal position of the curve is affected.

data acquisition and analysis are in principle straightforward, they still hold some pitfalls. First of all we have to deal with the so-called pixelization - a pronounced decorrelation of neighboring pixels on the one-pixel line due to the discretization of the microscopy images. In order to reduce this effect, we apply a cubic smoothing spline to the one-pixel-line thus allowing for coordinates which do not lie exactly on the grid which is given by the pixels of the image. Using a cubic spline ensures that (i) all given pixels on the filament's contour are included, (ii) the first and second derivative of the curve are continuous, and (iii) the fourth derivative of the position 
vector equals zero $\left(\frac{\partial^{4} \mathbf{r}(l)}{\partial l^{4}}=0\right)$. The latter constraint is equal to the equation of motion which minimizes the bending energy $H=\frac{\kappa}{2} \int_{0}^{L} d l\left[\frac{\partial^{2} \mathbf{r}(l)}{\partial l^{2}}\right]^{2}$. The Lagrangian density of the bending energy is

$$
\mathcal{L}\left(\mathbf{r}^{\prime \prime}\right)=-\frac{\kappa}{2} \mathbf{r}^{\prime \prime 2}
$$

and the Hamiltonian principle and the Euler-Lagrange equations yield

$$
\frac{d}{d t} \frac{\partial \mathcal{L}}{\partial \dot{\mathbf{r}}}-\frac{d^{2}}{d l^{2}} \frac{\partial \mathcal{L}}{\partial \mathbf{r}^{\prime \prime}}=\frac{\partial \mathcal{L}}{\partial \mathbf{r}} \Rightarrow \frac{\partial^{4} \mathbf{r}(l)}{\partial l^{4}}=0
$$

For confined filaments, the Hamiltonian includes a second energy term which, strictly speaking, should also be considered. However, we can apply the above argumentation since the segments of the filament in-between the wall contacts are still governed by bending energy.

Subsequently, equidistant points are placed on the smoothed line and these points are connected by secants. We assume that on small length scales the secants and the tangents are very similar and can be considered as equal. We choose a tangent length $l_{t}$ of $300 \mathrm{~nm}$ (corresponds to 4.8 pixels), since this is the approximate optical resolution of the system. We find that the vertical position of the tangent correlation function depends on the value chosen for $l_{t}$, as can be seen in figure 5.3. The same data set is analyzed in terms of the tangent correlation function for different values for $l_{t}$. The curve is vertically displaced to higher values for higher values of $l_{t}$. However, we have to keep in mind that the differences are very small: In the example shown here, decreasing $l_{t}$ by a factor of 25 decreases the values for $\langle\cos \theta(l)\rangle$ only by about $5 \%$. Also, the horizontal position of the curve and consequently the local minimum (indicated by the dashed line in figure 5.3) is not affected by the choice of $l_{t}$.

The microscopy and image processing procedures we use slightly broaden the contour due to restrictive optical resolution and thermal fluctuations during the exposure time of $100 \mathrm{~ms}$. The effect leads to a superposition of slightly different conformations of the filament in one and the same fluorescence micrograph (see figure 5.4). The full width at half maximum (FWHM) of a filament, or effective width, in an original gray scale micrograph is $D_{f} \approx 0.3-0.5 \mu \mathrm{m}$. After applying the appropriate threshold criterion to binarize the images, the filaments' effective width is $\sim 1 \mu \mathrm{m}$. Since the actual diameter of F-actin is merely $8 \mathrm{~nm}$, this implies that some higher frequency fluctuations cannot be visualized or analyzed. We account for this fact by introducing a correcting term in the theoretical description of the problem.[103] The local averaging of data over arc length distances similar to $D_{f}$ can be taken 


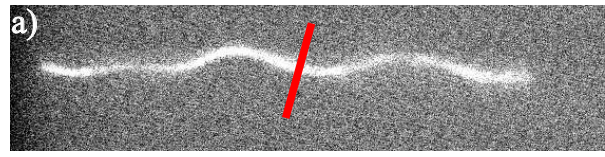

c)

b)
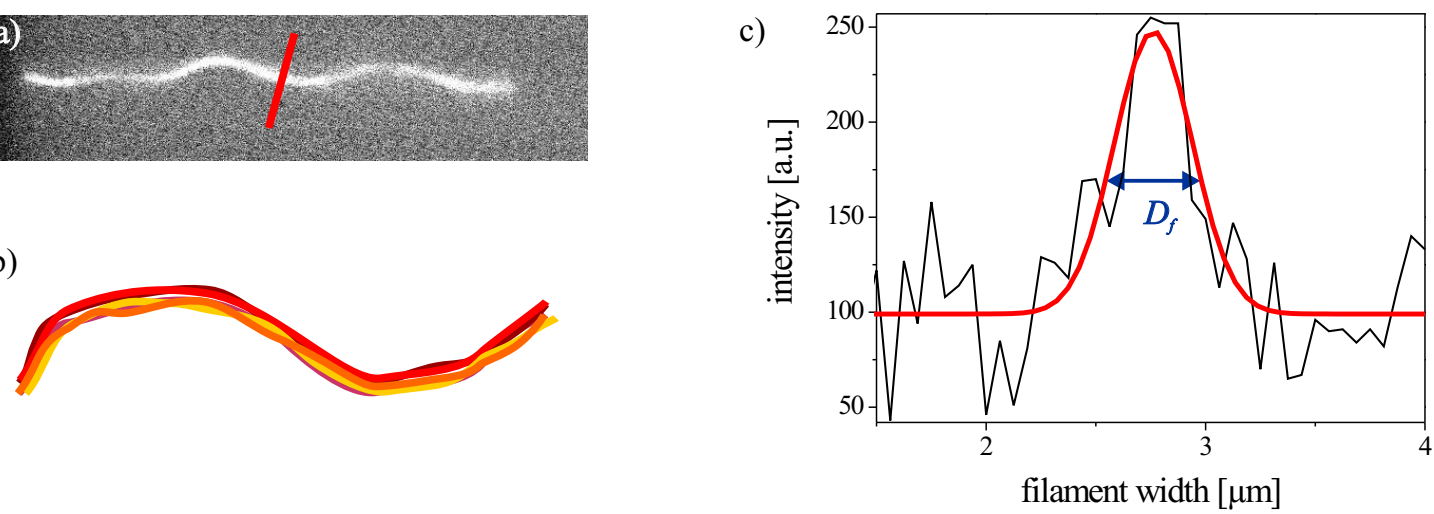

Figure 5.4: a) Micrograph and b) schematic representation of a slightly blurred actin filament owing to thermal fluctuations during the exposure time. c) Cross section and Gaussian fit (see red line in a) of the intensity in the micrograph. The effective width (FWHM) is $D_{f}=0.4 \mu \mathrm{m}$ in this case .

into account by convoluting the correlation function of Fourier modes (see equation 4.13)

$$
G(q)=\frac{k_{B} T}{\left(\kappa q^{4}+K\right)}
$$

with a Gaussian of width $\sim D_{f}$, similar to the Debye-Waller factor in solid state physics, which leads to

$$
G_{D}(q)=\exp \left(-q^{2} D_{f}^{2}\right) \frac{k_{B} T}{\left(\kappa q^{4}+K\right)}
$$

We use $D_{f}=0.5 \mu \mathrm{m}$ which is in agreement with the experimental values. The procedure is described in more detail elsewhere.[103] Fits of the data using equation 4.13 in consideration of equation 5.4 are performed using C-routines written by J. Kierfeld. [107] Thus, we are able to determine the free fitting parameters, the deflection length $\lambda$ and the persistence length $L_{P}$, and therefore to describe the mechanics of the filaments as well as the influence of the external potential. In order to improve the statistics, spatial averaging within the same image as well as thermal (that is to say, time) averaging, which includes many conformations of a filament, are performed. Discussing the data, we exclude the first few pixels $(\lesssim 1 \mu \mathrm{m})$ because it is extremely difficult, if not impossible, to make assumptions on a length scale similar to the optical resolution.[22] 


\subsection{From Free to Confined Filaments}

\subsubsection{Characterization of Unconfined Filaments}

The thermal fluctuations of unconfined filaments have been experimentally investigated intensively in the past (see also section 2.1).[21-27, 108, 109] Filaments are restricted between two glass slides and observed in the focal plane of the microscope. $L_{P}$ is derived by different methods which leads to values between $7.4-22 \mu \mathrm{m}$. The tangent correlation functions show the exponential decay as theoretically predicted, with $L_{P}$ as the characteristic length scale (see equation 4.3).

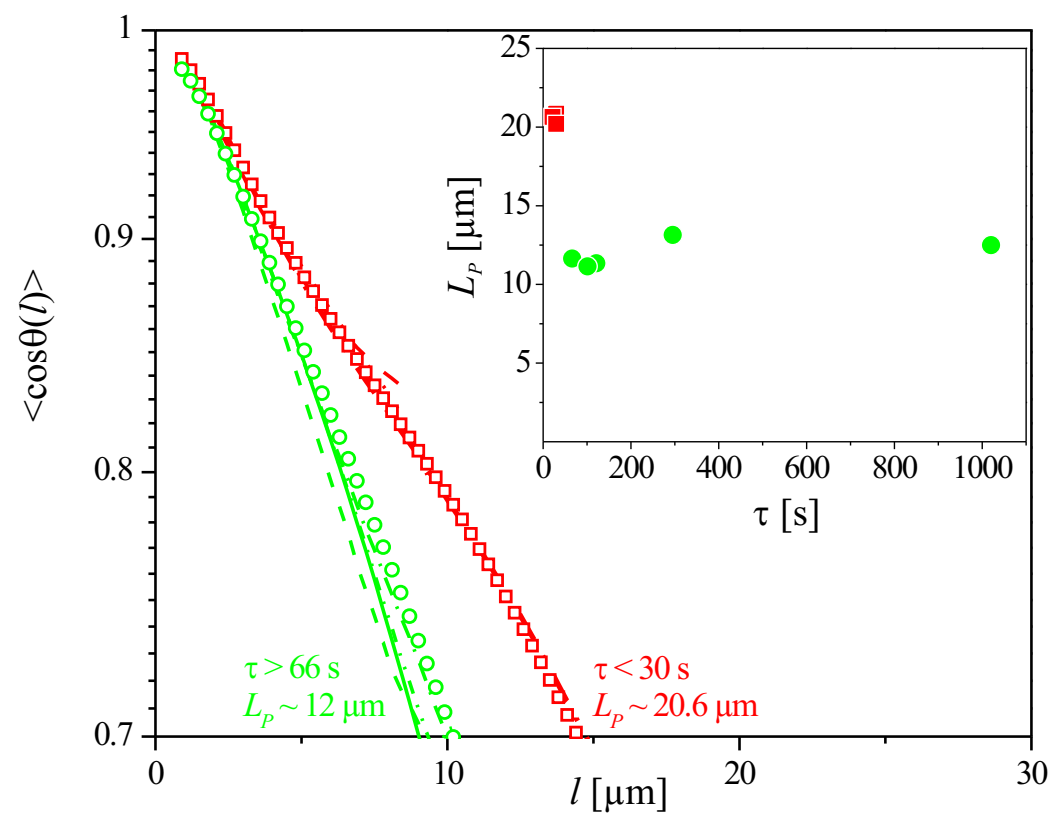

Figure 5.5: Tangent correlation functions of individual, unconfined filaments. Red lines and symbols: filaments with a total observation time $\tau<30 \mathrm{~s}$. Green lines and symbols: filaments with a total observation time $\tau>66 \mathrm{~s}$. Inset shows a plot of $L_{P}$ against $\tau$. Clearly, if $\tau$ is too short, filaments seem to be stiffer than they are.

In order to characterize our specific actin system, we also analyze measurements with filaments that freely fluctuate in a quasi two-dimensional environment between two glass surfaces without further lateral confinement. We find a dependence of $L_{P}$ on the total observation time $\tau$. The red and green data lines and symbols in figure 5.5 show semi-logarithmic plots of such tangent correlation functions of unconfined filaments. The red lines represent data sets derived from filaments where the total observation time is $\tau \leq 30 \mathrm{~s}$. The characteristic decay $L_{P}$ of the exponential function is $20-21 \mu \mathrm{m}$ (see red squares in inset). In contrast, if the total observation time 
is increased to $\geq 66 \mathrm{~s}$ (green lines), $L_{P}$ is decreased to $11-13 \mu \mathrm{m}$ (see green circles in inset). Even for $\tau>17 \mathrm{~min}=1020 \mathrm{~s}$ the value of $L_{P}$ is not decreased any further. After an observation time as short as $30 \mathrm{~s}$ the filament has not taken all possible shapes yet. That is to say, the complete conformational space of the system has not been occupied and the individual snapshots of the filaments' contour are still very correlated. For $30 \mathrm{~s}<\tau<66 \mathrm{~s}$ we expect a smooth transition in the value of $L_{P}$. Our data indicate that after about 1 min observation time the filament has taken all possible conformations and $L_{P}$ reaches an asymptotic value of $12 \pm 0.8 \mu \mathrm{m}$.

To support this assumption, we compare different total observation times for one specific filament. The altogether 2360 individual snapshots of the filament are taken in subsets of 295 images each, with an exposure time of $100 \mathrm{~ms}$ and no delay time in-between the images. Between the subsets there is a delay time of $1-3 \mathrm{~min}$ and the total observation time of the filament adds up to more than 17 min. First, only one data subset (295 images, $\tau=29.5 \mathrm{~s}$ ) is included in the analysis that leads to a tangent correlation as shown by the red open squares in figure 5.5 and $L_{P}=20.2 \mu \mathrm{m}$. Second, we analyze every tenth image of the whole data set, so that the total number of images (240) is approximately the same as in the first case, but the total observation time is an order of magnitude higher $(\tau \geq 17 \mathrm{~min})$. We obtain the tangent correlation shown by the green open circles and $L_{P}=12.5 \mu \mathrm{m}$. Obviously, to deduce the real $L_{P}$ of the data it is therefore crucial to observe the individual filaments over a sufficiently long period of time. It is not primarily the mere number of images included into a data set which improves the statistics of the measurement but rather the prominent role of $\tau$. Actin filaments that are confined in microchannels are much more sensitive to breaking than unconfined filaments. Mostly, we recorded data for $\sim 30$ s which proved to be sufficiently long for the filament to thoroughly occupy the conformational space, which is smaller if a microchannel is employed than in the unconfined case.

\subsubsection{Tangent Correlation Function of Confined Filaments}

Confinement of F-actin is achieved by placing the filaments into rectangular channels of varying widths and geometries. We consider our system two-dimensional for the following two reasons. First of all, the depth $h$ of the microchannels for all experiments presented here is small $(\leq 1.4 \mu \mathrm{m})$. Monte Carlo simulations show that a further reduction of the channel depth does not influence the results. [107] Secondly, using optical microscopy, as a matter of principle we observe the molecules in the focal plane. Possible fluctuations that occur in the third dimension cannot 


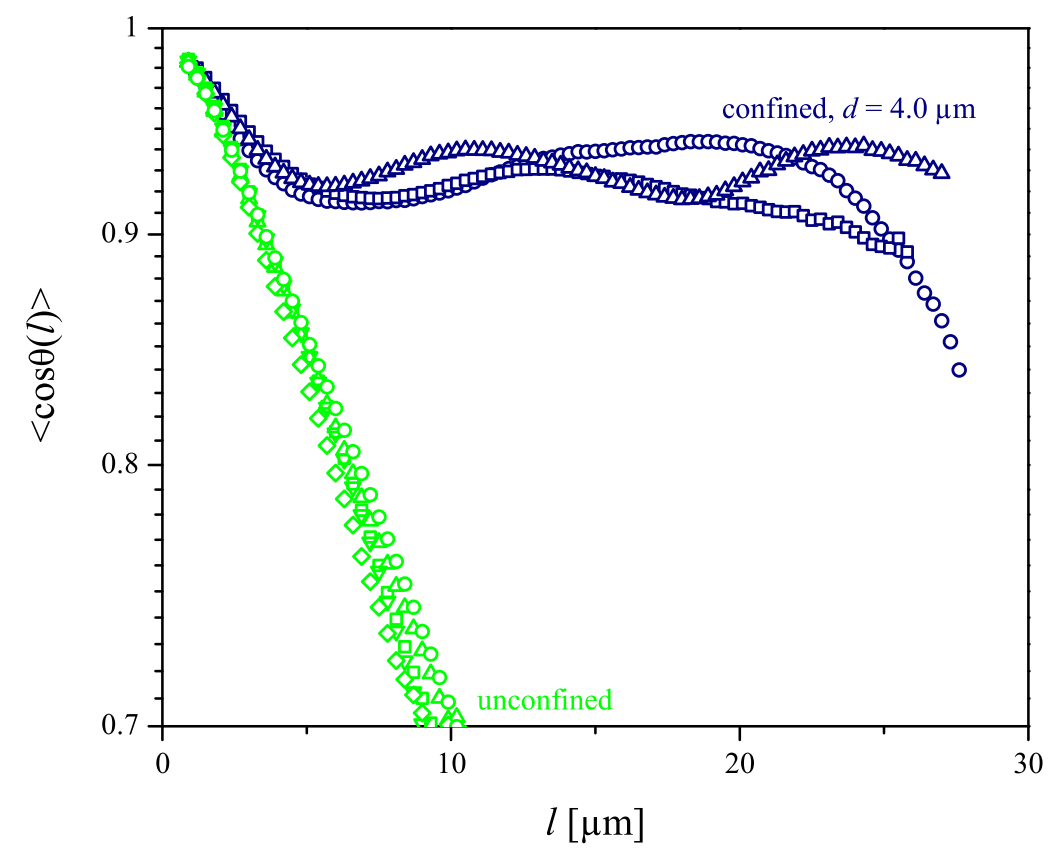

Figure 5.6: Tangent correlation functions of individual filaments. Blue symbols: confined filaments, channel width $d=4.0 \mu \mathrm{m}$. Green symbols: unconfined filaments. Despite of individual deviations the increased overall correlation of the confined filaments is clearly visible.

be visualized. Since the perpendicular directions decouple from each other, we can disregard the third dimension.

In simulations, the fluctuations of the filaments and the corresponding tangent correlation functions in three dimensions can be investigated. This results in more detailed information about the system. It has been shown that for filaments confined between two walls with distance $h$ there is a crossover between the twoand the three-dimensional case. [110] For arc length distances $l$ smaller than $h$ the filaments show three-dimensional behavior and the tangent correlation function $\langle\cos \theta(l)\rangle_{f}=\exp \left(-l / L_{P}\right)$ is obtained, whereas for $l>h$ two-dimensional behavior is observed, that is to say, the tangent correlation is $\langle\cos \theta(l)\rangle_{f}=\exp \left(-l / 2 L_{P}\right)$. If the simulated chain is projected onto a two-dimensional plane before the tangent correlation function is determined, which corresponds to the microscopy experiments, there is no such transition and the two-dimensional behavior is present for the complete range of $l$. This result is in good agreement with our experimental observations. We can therefore equate our system with the two-dimensional case and apply the corresponding analytical description.

In figure 5.6, tangent correlation functions of confined (blue symbols) and unconfined (green symbols) filaments are compared with one another. The channel width 
is $d=4.0 \mu \mathrm{m}$. Despite individual variations within the two groups of data (free versus confined filaments) the completely altered course of the tangent correlation function under the influence of confinement is obvious. The most striking feature is that the overall correlation is dramatically increased. Instead of reaching a zero correlation for infinitely large distances $l$, as in the unconfined case, the curves approach an asymptotic value. The fluctuations about the saturation plateau, which are clearly observed here and which vary individually for each of the three data sets (that is to say, filaments) shown here, can be attributed to slight differences between the individual macromolecules. They are indeed averaged out once several data sets are included.

The ramification of our observation is that the tangential orientations of two polymer segments which are far apart from each other (large $l$ ) seem to be very well correlated (in contrast to the free tangent correlation, where they are not at all correlated). However we have to keep in mind that this is not a direct coupling of the segments as in the case of stiffer polymers with larger persistence lengths. All segments are coupled to the external channel potential and thus merely indirectly coupled to each other. The external confining potential thus serves as a micrometerscale track for the polymer. As a matter of principle there is a noteworthy difference: The tangent correlation function of stiffer polymers that are freely fluctuating without any confining microchannels (for example, microtubules with $L_{P} \sim \mathrm{mm}$ ) still shows an exponential decay, but $L_{P}$ is larger and thus the decay is weaker. On the contrary, here we observe a characteristic oscillation along the tangent correlation function whose wavelength is set by the external potential.

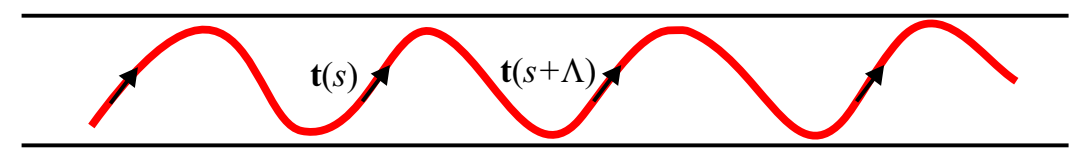

Figure 5.7: Schematic representation of an idealized polymer chain which is periodically reflected at the channel walls. The tangential orientation, indicated by the arrows, returns to its original direction after one wave length $\Lambda$.

In a simplified manner, we can imagine a periodic course of the contour of the filament. In this idealized case the tangential orientation of the polymer's contour would return to its initial value after one wave length $\Lambda$ (see figure 5.7). Therefore, the correlation between these parallel vectors would be 1 and the numerical value of the correlation function would oscillate between a minimal value and 1. Naturally, in reality the filament does not follow such an ideal course, rather the deflections on each channel wall are superimposed by the intrinsic thermal fluctuations caused by 
Brownian motion of the biopolymer. Consequently, the correlation does not thoroughly return to 1 . Since the value of $\Lambda$ is determined by the channel width $d$, we will discuss the influence of confinement in more detail in the following section.

\subsubsection{Influence of Contour Length and Channel Width}

In figure 5.8 tangent correlation functions of individual actin filaments with varying contour lengths $(L=18-51 \mu \mathrm{m})$ fluctuating in channels of different widths $(d=1.5 \mu \mathrm{m}, 4.2 \mu \mathrm{m}, 5.8 \mu \mathrm{m})$ are shown. Each data set represents one individual filament and is averaged over all conformations of this filament. The principle course is the same for all data sets. For small values of $l \lesssim 5 \mu \mathrm{m}$ we observe a decay which leads to a pronounced minimum, followed by oscillations. The length of this oscillation regime depends on the length of the filament. The oscillations are followed by a strong decay, that is to say, a dramatic decrease of the correlation. This quasi-free behavior on a length scale on the order of the channel width is due to the finite length of the filaments. The free ends of the filaments are not distinctly influenced by the channel walls and thus show free behavior. Even though in the examples shown here the number of conformations is rather small for the smaller channels $(d=1.5 \mu \mathrm{m})$ it can clearly be seen in figure 5.8 that the statistics are better. The data sets show less individual variations than for larger $d$ and the course of the tangent correlation is more defined. This can be attributed to the fact that in this case the conformational space of the system is smaller and is occupied in shorter time spans $\tau$.

A further improvement of the statistics is achieved by averaging data sets derived from several filaments and thus eliminating features that stem from specific individual differences. In figure 5.9 semi-logarithmic plots of the tangent correlation functions for fluctuating filaments in microchannels of different widths $(d=1.5 \mu \mathrm{m}$, $4.2 \mu \mathrm{m}, 5.8 \mu \mathrm{m})$ are shown. $L=28-51 \mu \mathrm{m}$ is chosen for this analysis to ensure that the contour length of the filaments is large compared to the channel width $d$. Compared to the data shown in figure 5.8, the oscillations about the saturation level are averaged out. This fact enables us to describe the curve in more detail and compare it to the analytical expressions discussed in section 4.3. The dashed line marks the ideal tangent correlation of a freely fluctuating filament $\left(L_{P}=12 \mu \mathrm{m}\right)$ in two dimensions, while the open symbols show experimental results for filaments in channels. Clearly, filaments confined in $9.8 \mu \mathrm{m}$ channels $(L \approx 3 d)$ are not very strongly influenced by the channel geometry; they show a behavior similar to free filaments. However, for the smaller channels $(d \leq 5.8 \mu \mathrm{m})$ a strong influence of the 
restraining channel walls is observed. We observe an overall increasing correlation with decreasing channel width. For large $l$ the correlation reaches a large asymptotic value of $1-\lambda /\left(4 L_{P}\right)$, instead of dropping to zero as for unconfined filaments. This saturation plateau increases with decreasing channel width, whereas the position of the local minimum $l_{\text {min }}=\pi \lambda / 2$ is shifted to smaller values with decreasing channel width.

The averaged data sets shown in figure 5.9 as well as the individual data sets included in the averaging are fitted separately in order to determine the two free fit parameters $\lambda$ and $L_{P}$. We use equation 4.21 in consideration of the correction factor introduced in equation 5.4, as explained in section 5.1. The fit curves of the averaged data sets are shown by solid lines in figure 5.9. Since data for very large arc length distances $l$ are generally less statistically reliable, we have excluded those values from the fits. Clearly, the fit for the smallest channels $(d=1.5 \mu \mathrm{m})$ is best. In the WLC model, infinitely long filaments are assumed. This approximation is best realized for a high ratio of filament length to channel width $(>18$ in the case of the $1.5 \mu \mathrm{m}$ channels).

In figure 5.10 the results for $L_{P}$ are plotted against $d$. The closed red circles are the values deduced from the averaged data curves, whereas the open red circles mark the values for the individual data curves. The error bars have been determined as follows: $\Delta d=0.2 \mu \mathrm{m}$, corresponding to the optical resolution of the microscope used to measure the channel width and $\Delta L_{P}$ is the standard deviation of the data for individual filaments. We are able to show that all values for $L_{P}$ lie in the range in-between $\sim 10$ and $20 \mu \mathrm{m}$, which is in agreement with values measured for unconfined filaments. The scatter of the values is smaller for smaller channel widths, which again can be attributed to the smaller conformational space for smaller channels. The dashed line corresponds to $L_{P}=(13.1 \pm 0.6) \mu \mathrm{m}$, which is the mean of all values for $L_{P}$ determined here.

The results for the second fit parameter $\lambda$, which is characterized by the channel wall potential, are plotted logarithmically against $d$ in figure 5.11. Once again, the open red circles correspond to data from individual filaments and the closed red circles to the averaged data. The error bars have been determined as follows: $\Delta l_{\text {min }}$ is the error of reading the data and $\Delta \lambda$ is the standard deviation as calculated by the scattering of the values for the individual data sets.

We are aware of the fact that the tangent correlation reacts sensitively to parameters used for data analysis such as the digitalization of the contour of the filaments, smoothing due to application of image processing filters, and the choice of the tangent vector length $l_{t}$ (see discussion in section 5.1 ). Thus, we perform a consistency 


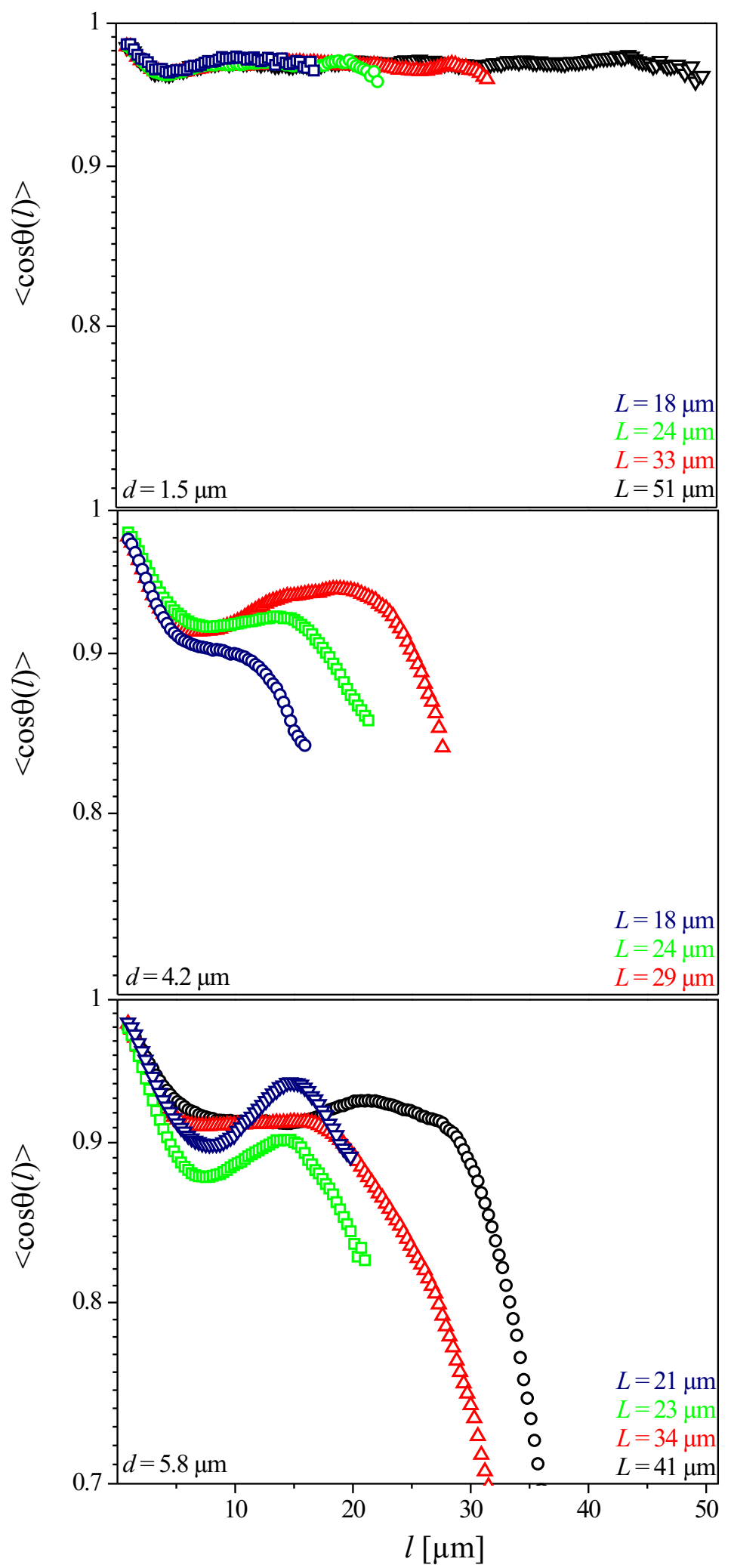

Figure 5.8: Tangent correlation functions of individual actin filaments of different contour lengths $L$ confined in channels of different widths $d$. 


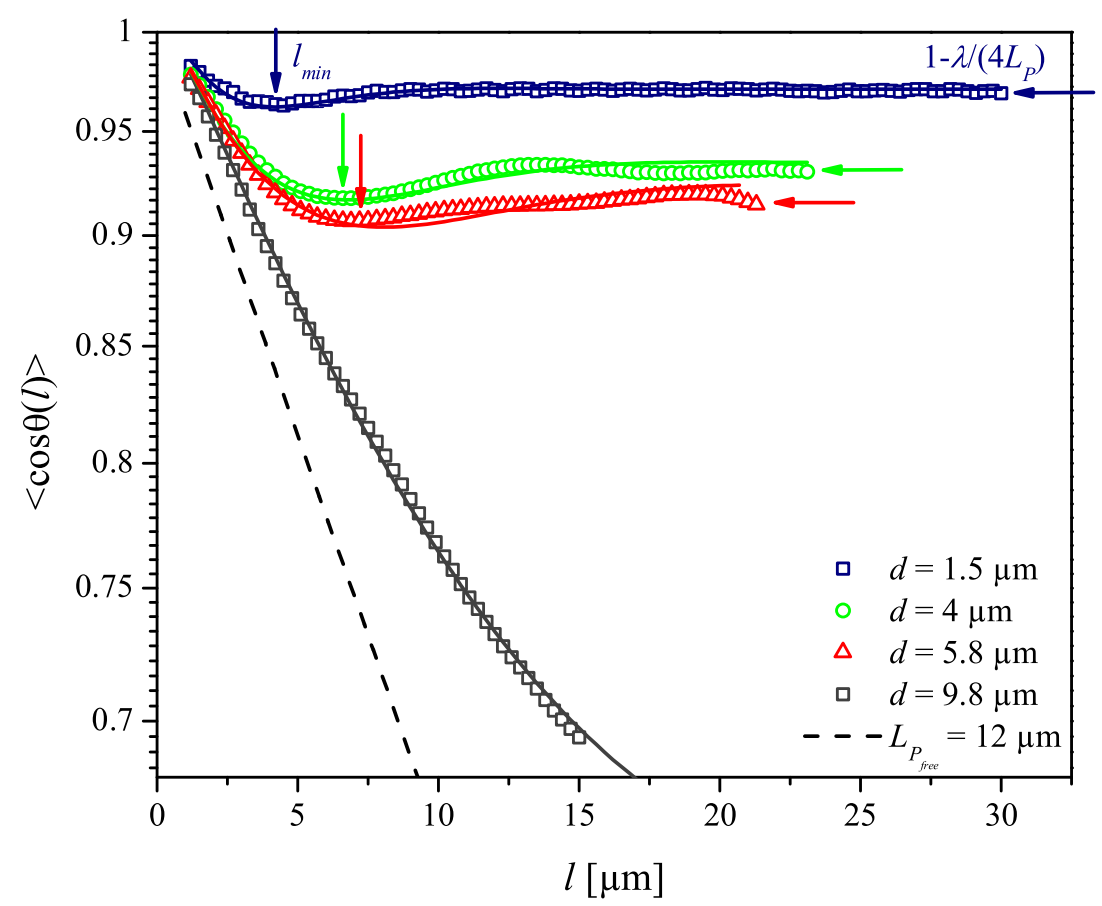

Figure 5.9: Averaged tangent correlation functions of actin filaments confined in channels of different widths $(1.5-9.8 \mu \mathrm{m})$. The contour length of the filaments included in the averaging is $28-51 \mu \mathrm{m}$. Open symbols: experimental data; solid lines: fit curves; dashed line: ideal tangent correlation function of free filaments. Decreasing channel width $d$ increases the overall correlation of the filaments' contour. Arrows denote $l_{\min }$ and the saturation plateau.

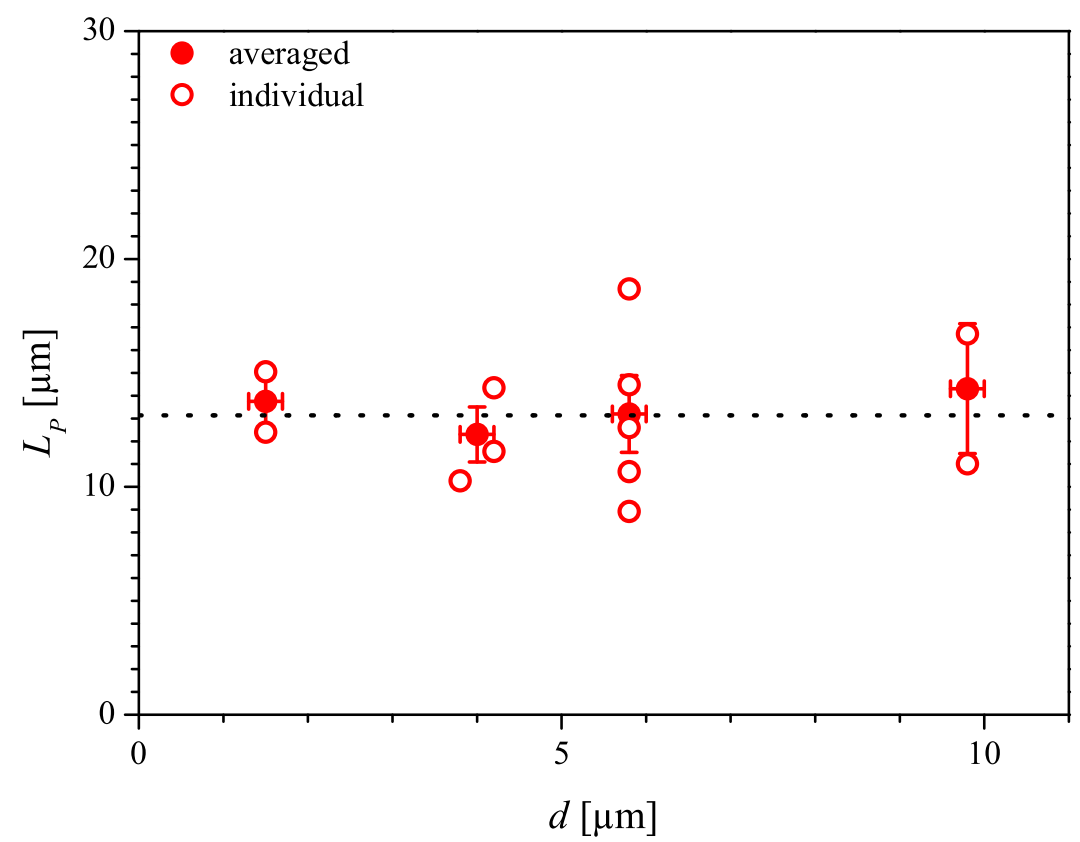

Figure 5.10: The persistence length $L_{P}$ plotted against the channel width $d$. Dashed line: mean value $L_{P}=13.1 \mu \mathrm{m}$. 


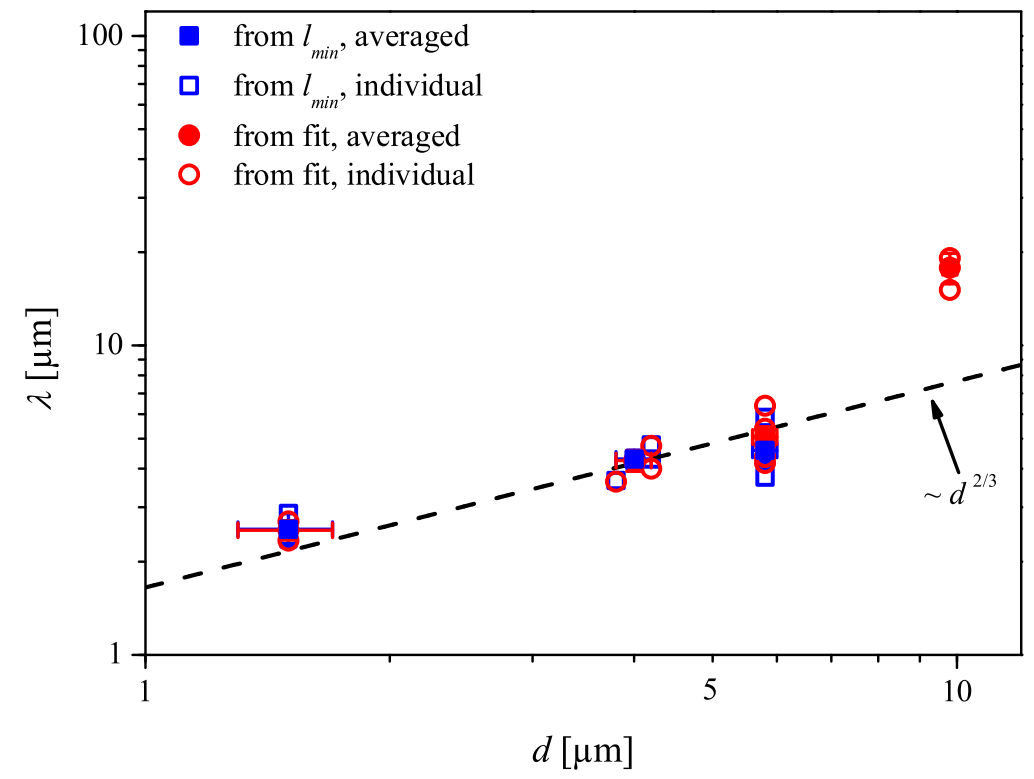

Figure 5.11: The deflection length $\lambda$ plotted against the channel width $d$. The Odijk scaling $\lambda \propto d^{2 / 3}$ (dashed line) is experimentally confirmed. Filaments in $9.8 \mu \mathrm{m}$ channels are not distinctly confined.

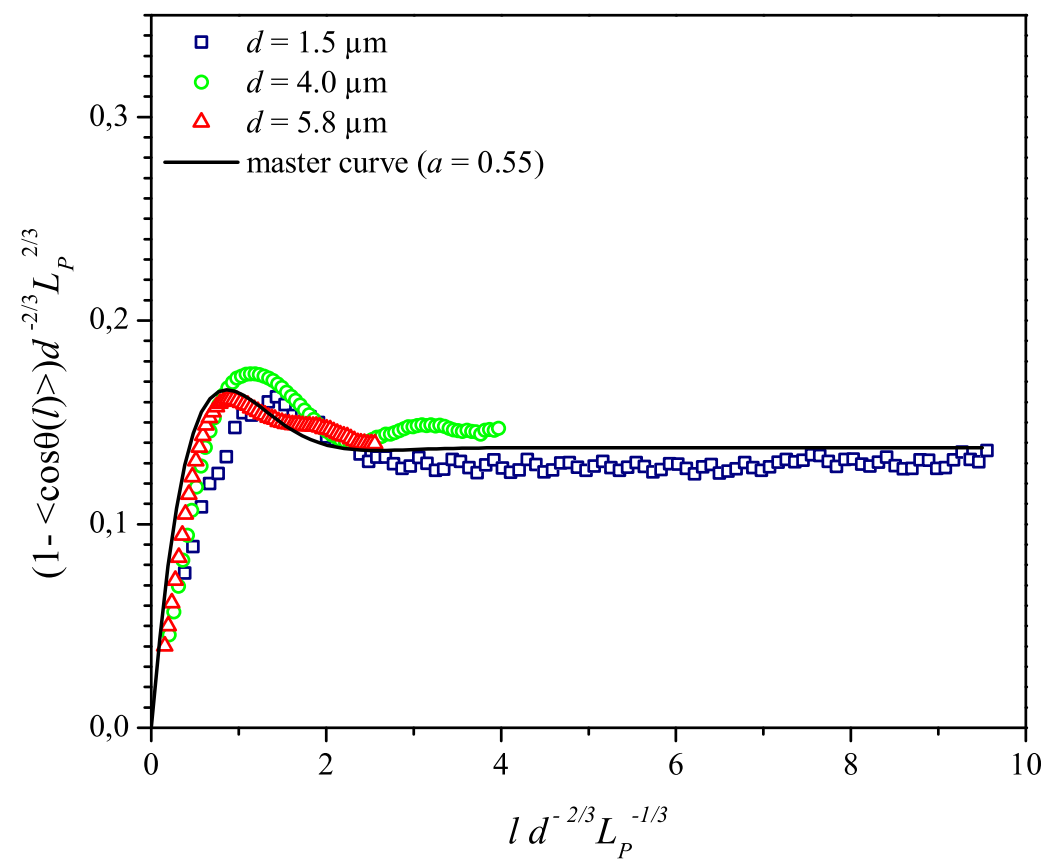

Figure 5.12: Rescaled tangent correlation functions for three different channel widths (open symbols). The solid black line marks the master curve on which all data collapse upon rescaling, $a=0.55$. 
check of both our experimental results and the model used for data analysis. In section 4.3 we have seen that the position of $l_{\text {min }}$ is determined exclusively by the value of $\lambda$, while, on the other hand, the horizontal position of the tangent correlation function is not affected by changes of the parameters used for analysis. Therefore, the local minima of the averaged data curves (figure 5.9) as well as the data curves corresponding to individual filaments are determined. Using equation 4.26, values for $\lambda$ are obtained. These data points are shown by the blue squares in figure 5.11 , where the open symbols correspond to data from individual filaments and the closed symbols represent the averaged data. The data for the $9.8 \mu \mathrm{m}$ channels do not display a local minimum because the confining potential is relatively weak. The values of $\lambda$ for channel widths $d=1.5 \mu \mathrm{m}, 4.0 \mu \mathrm{m}$, and $5.8 \mu \mathrm{m}$ are in good agreement for both methods of analysis, which shows that we have chosen stable parameters for our data analysis.

In addition, in figure 5.11 the Odijk scaling law $\lambda \propto d^{2 / 3}$ is shown (equation 4.16, dashed line). For $d \leq 5.8 \mu \mathrm{m}$ the experimental results agree very well with the scaling law whereas the value for $d=9.8 \mu \mathrm{m}$ is not in agreement with the scaling law. This result supports the above mentioned assumption that filaments in wide channels are merely weakly confined. The scaling law is confirmed, however, the coefficient differs from what has been calculated for circular tubes and a hard wall potential.[43] This discrepancy may be attributed to approximating the confining geometry by a parabolic potential. In turn, the shape and structure of the confining potential plays an important role in setting $\lambda$ as a characteristic length scale. The parabolic potential we used for the model is a simple but effective estimate.

The scaling law relating $d, L_{P}$, and $\lambda$ suggests that the data curves derived from measurements in different channels can be rescaled and should collapse on a single master curve. To prove this assumption equation 4.21 is rescaled by $d^{-2 / 3} L_{P}^{2 / 3}$

$$
(1-\langle\cos \theta(l)\rangle)_{c}^{*}=\left(1-\langle\cos \theta(l)\rangle_{c}\right) d^{-2 / 3} L_{P}^{2 / 3}
$$

The function is plotted over the arc length distance rescaled by $d^{-2 / 3} L_{P}^{-1 / 3}$ such that

$$
l^{*}=l \cdot d^{2 / 3} L_{P}^{1 / 3}
$$

The resulting master curve

$$
\left(1-\left\langle\cos \theta\left(l^{*}\right)\right\rangle\right)_{c}^{*}=\frac{a}{2 \sqrt{2}}\left(\cos \left(\frac{\pi}{4}\right)-\cos \left(\frac{\pi}{4}+\frac{l^{*}}{a}\right) \exp \left(\frac{-l^{*}}{a}\right)\right)
$$

is independent of $L_{P}$ and $\lambda$ and only contains the coefficient $a$ in the Odijk scaling in equation 4.16. The rescaled data curves are shown in figure 5.12, When fitting the 
three data curves for $d=1.5 \mu \mathrm{m}, 4.0 \mu \mathrm{m}$, and $5.8 \mu \mathrm{m}$ independently with equation 5.7, we obtain $a=0.52,0.55$, and 0.57 , respectively. In figure 5.12, the solid black line denotes the master curve for the mean value, $a=0.55$.

In addition to the analytical description, our data shall be compared to results obtained by simulating the system. J. Kierfeld has performed Monte Carlo simulations for semiflexible polymers in rectangular channels with $h \ll d$ such that the quasi two-dimensional case was realized. All parameters were chosen comparable to the experiments discussed here for this procedure, which is described in more detail elsewhere.[103] Simulations for different $L_{P}$ and $d$ have been performed. Since the master curve described by equation 5.7 unifies the characteristic length scales of the system in one single parameter $a$ it provides a very well suited method to compare experiment and simulation. For the simulations $a=0.59$ is obtained, which is in good agreement with our experimental results.

The experimental system is characterized by the bending energy of the filaments, given by $L_{P}$, and the confining channel potential, given by $\lambda$. Both parameters can consistently be derived from the experimental data, and the results agree with the simulations as well as the analytical description taking into account the channel potential as parabolic. Somewhat surprising, the ad hoc assumption to approximate the rectangular hard wall potential by a simple parabolic potential works exceedingly well.

In order to allow for direct comparison of the mechanical properties of F-actin with those of other materials, we calculate the Young's modulus $E$ using

$$
E=\frac{L_{P} k_{B} T}{I}
$$

where $I$ is the geometrical moment of inertia. The numerical value for actin is $I=2.8 \cdot 10^{-35} \mathrm{~m}^{4},[25]$ which leads to $E=1.9 \mathrm{GPa}$. This is comparable with annealed tantalum with a Young's modulus of $E=1.83 \mathrm{GPa}$, or nickel, $E=2.02 \mathrm{GPa}$. To put these values in perspective, steel wire has $E=196 \mathrm{GPa}$.[111] The value of $E$ for actin is quite large, considering that it is a biomaterial. On the other hand, it may partly explain the remarkable strength of muscles.

To better ascertain which energy $W$ is acting on the filaments evoked by the wall potential, we plot the parabolic term $K / 2 \cdot z(x)^{2}$ (red solid line in figure 5.13) as well as the corresponding hard wall potential (black solid line) for $d=4.0 \mu \mathrm{m}$. $W$ per $\mu \mathrm{m}$ is plotted in units of $k_{B} T$. We estimate the potential energy directly at the channel wall, that is to say at $z=d / 2=2 \mu \mathrm{m}$. We use $L_{P}=13.1 \mu \mathrm{m}$ and $\lambda=4.25 \mu \mathrm{m}$ :

$$
\frac{K}{k_{B} T}=\frac{\sqrt{2} L_{P}}{\lambda^{4}} \Rightarrow W(2 \mu \mathrm{m})=\frac{\sqrt{2} L_{P}}{2 \lambda^{4}} \cdot(2 \mu \mathrm{m})^{2} k_{B} T=0.114 k_{B} T \mu \mathrm{m}^{-1}
$$




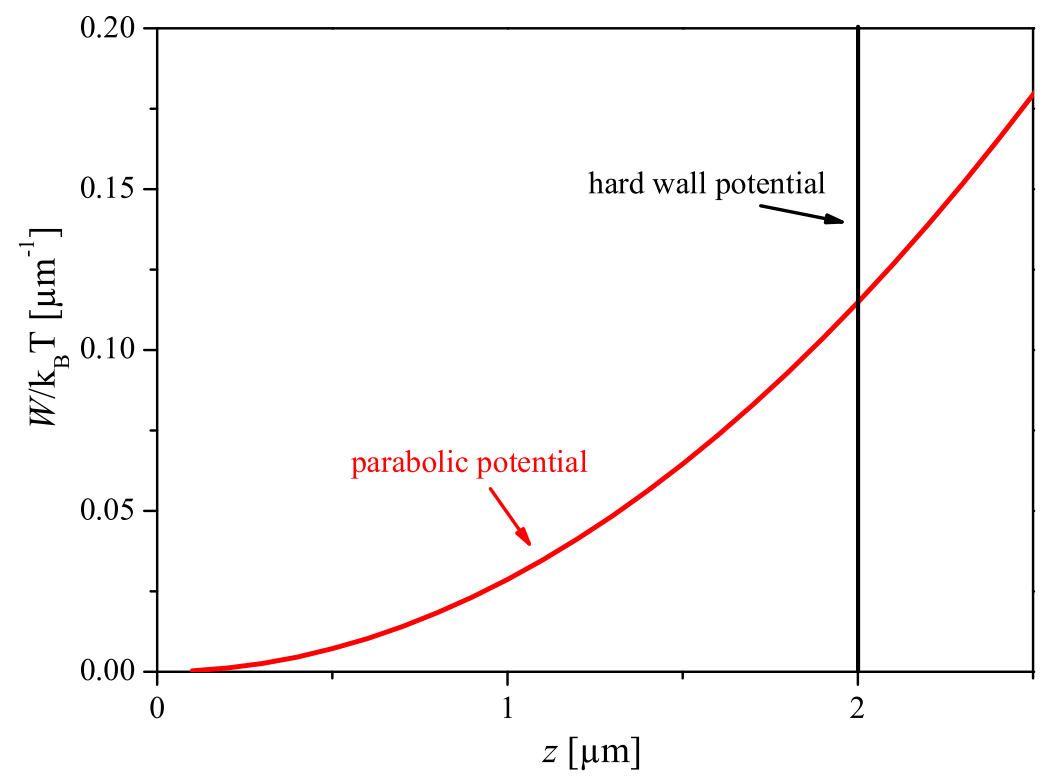

Figure 5.13: The parabolic potential energy per $\mu \mathrm{m}$ in units of $k_{B} T$ plotted against the $z$-coordinate perpendicular to the channel direction (red solid line). For comparison the hard wall potential for the corresponding $d=4.0 \mu \mathrm{m}$ is plotted (black solid line).

Thus, the potential energy per $\mu \mathrm{m}$ of filament length is about one order of magnitude smaller than the thermal energy $k_{B} T$. If we consider a filament with a length of $L=20 \mu \mathrm{m}$ the total potential energy at the channel wall is $2.28 k_{B} T$. A segment of the filament has to raise its thermal energy remarkably, on the order of magnitude of $10^{-1} k_{B} T$ per $\mu \mathrm{m}$, so as to approach to the channel wall. In contrast, for a real hard wall potential the potential energy is absolutely zero inside the channel and increases to infinity only at the channel wall. In consideration of this fact, is is all the more impressive that we can describe our experiments so accurately by approximating the confining energy by a parabolic potential.

\subsection{Radial Distribution Function}

Apart from the tangent correlation, the radial distribution function is an important statistical measure in polymer science. Experimentally, it might be easier to obtain only specific points along the filaments' contour rather than the whole contour line. However, small imperfections or defects that yield kinks in the shape of the polymer have a strong influence on the global radial distribution function, while they tend to average out for the local tangent correlation function. In addition, data sets as shown in figure 5.14 give rise to 200 - 300 individual data points, while for the tangent correlation functions (as presented earlier in this chapter) the spatial averaging 


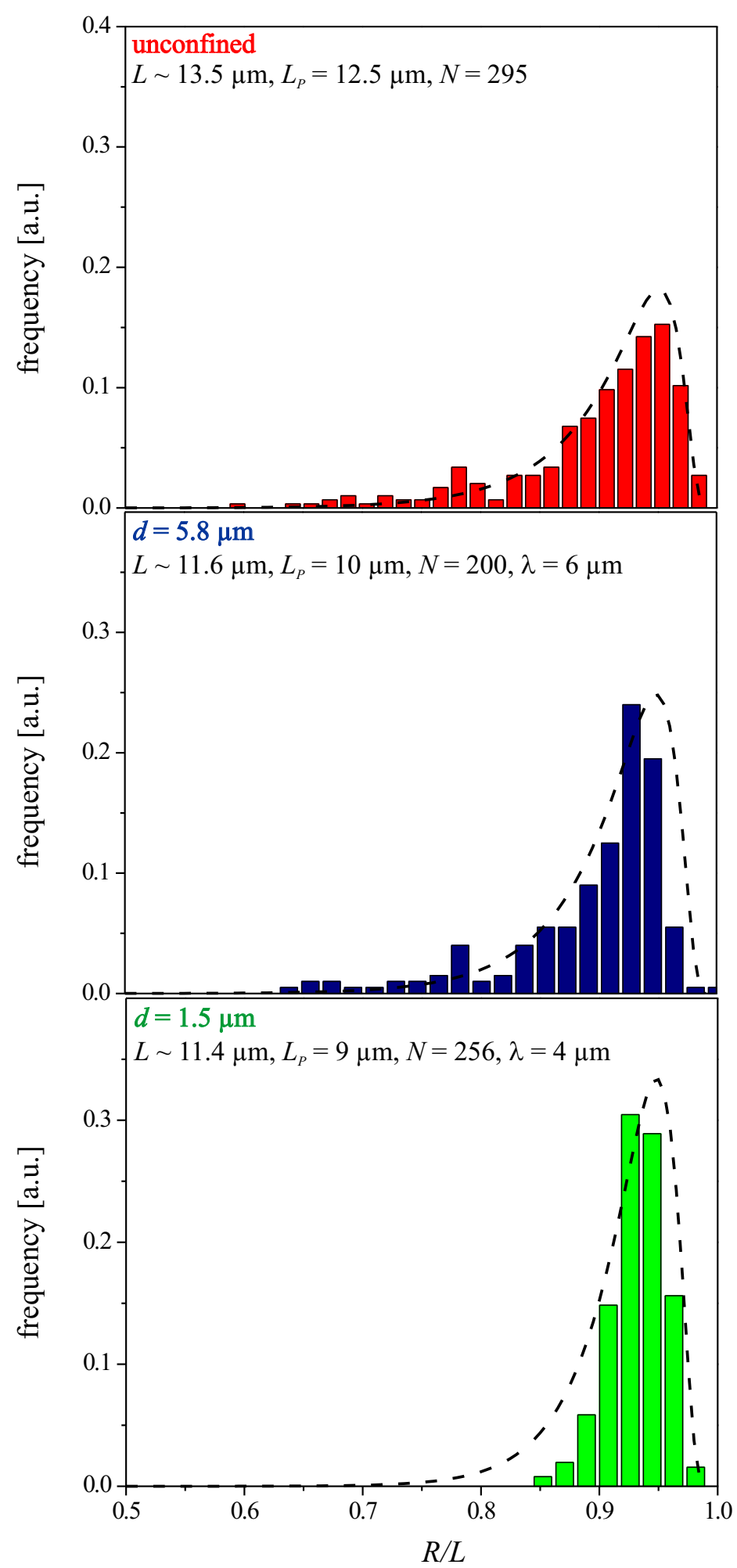

Figure 5.14: Radial distribution function of a free, a modestly confined $(d=5.8 \mu \mathrm{m})$, and a strongly confined $(d=1.5 \mu \mathrm{m})$ individual actin filament. The results can be fitted using equations 4.8 and 4.27 (dashed lines). 


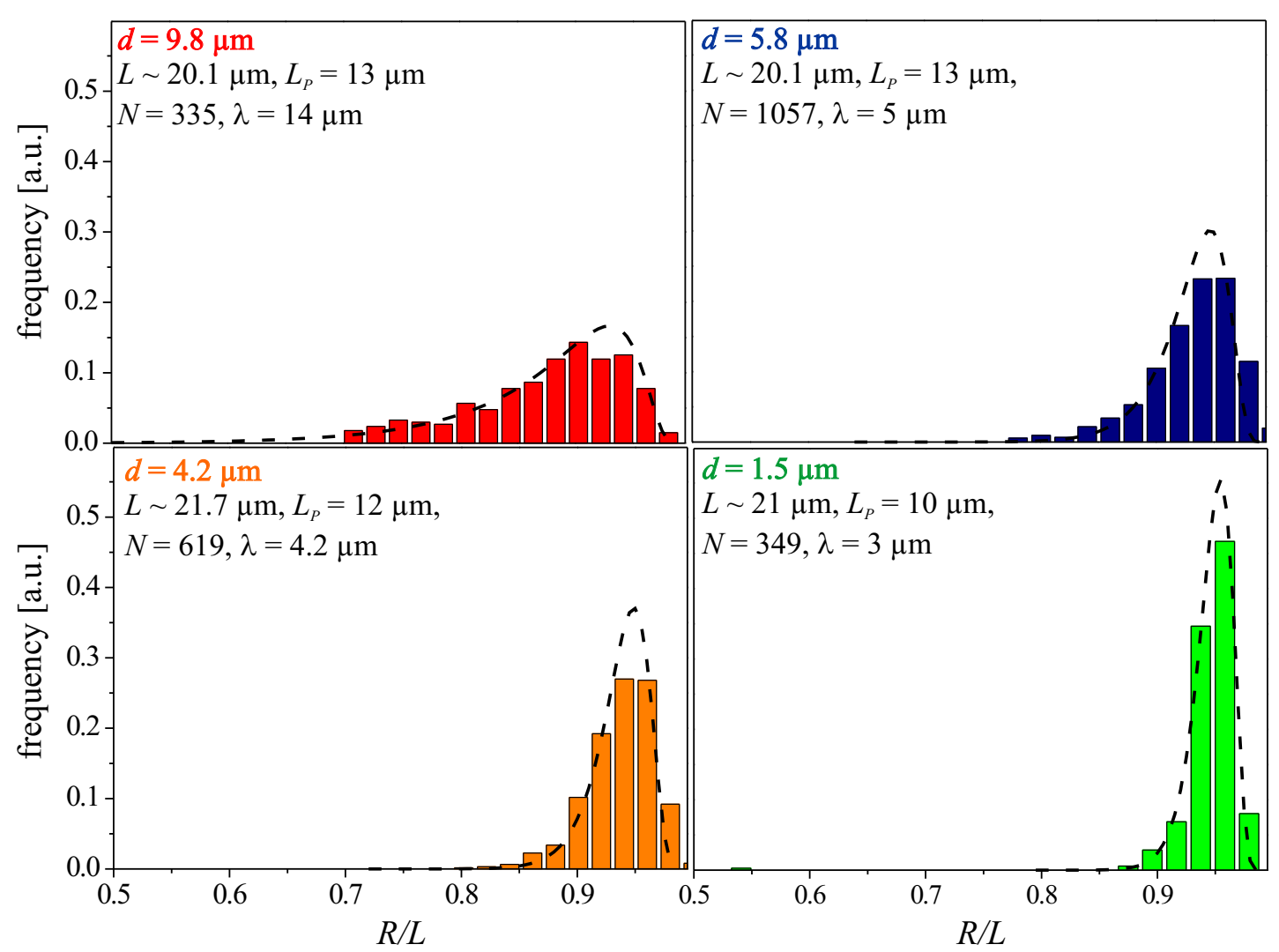

Figure 5.15: Radial distribution function of confined actin filaments in channels of different widths. The data have been combined from several individual filaments. Dashed lines: fits using equation 4.27 .

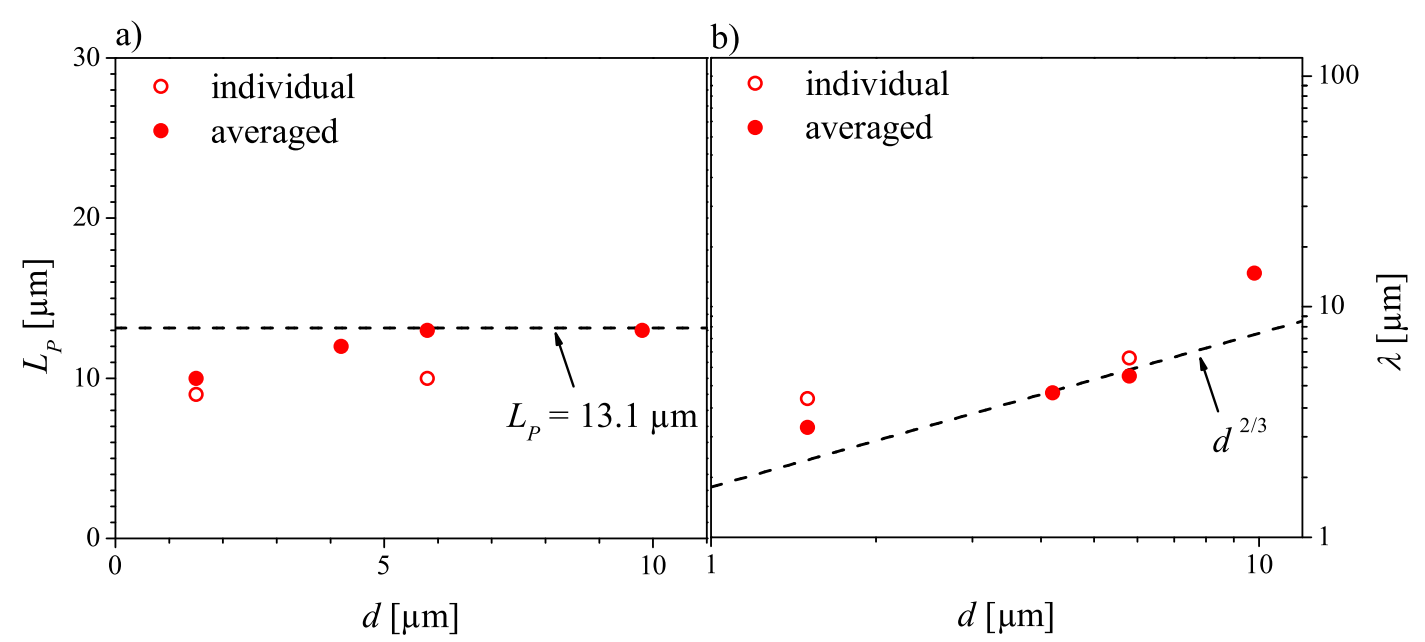

Figure 5.16: a) $L_{P}$ plotted against $d$. Dashed line: mean value derived from analysis of the tangent correlation function. b) $\lambda$ plotted against $d$. Dashed line: Odijk scaling, parameters chosen as in figure 5.11 . 
within one image of a filament yields additional statistical weight.

In figure 5.14 the normalized radial distribution functions for a free, a modestly confined $(d=5.8 \mu \mathrm{m})$, and a strongly confined $(d=1.5 \mu \mathrm{m})$ actin filament are shown. Each data set stems from one single actin filament and we cover 200 to 300 conformations. We plot the frequency of a certain end-to-end distance $R$ against the normalized value of $R / L$. In our case, $R$ is the distance between the end points of the filament and $L$ is the arc length between them. The histograms are normalized such that the sum of all frequencies is 1 . The step size for the histograms is chosen such that a range corresponding to the approximate optical resolution is covered $(210 \mathrm{~nm})$. The statistics of our results may be moderate in comparison to the data shown in figure 5.9, but nevertheless the results are in good agreement with the theoretical description. This is emphasized by the fit curves (dashed lines) that show the course described by equations 4.8 and 4.27 . When the filament is confined, the distribution function narrows and the maximum is shifted to larger values of $R / L$. In order to improve the statistics of the data analysis we have combined data of several filaments. It is important to ensure that the data sets stem from filaments having approximately the same length in a channel of equal width, because both parameters determine the course of the curve. In figure 5.15, filaments with $L \sim 20 \mu \mathrm{m}$ are compared for different channel widths $(d=9.8 \mu \mathrm{m}, 5.8 \mu \mathrm{m}, 4.2 \mu \mathrm{m}, 1.5 \mu \mathrm{m})$. The data clearly show that a stronger confinement (smaller $d$ ) leads to a narrower distribution function.

For all histograms shown here, fits with the corresponding equations yield values for $\lambda$ and $L_{P}$ that are in good agreement with the values deduced from the tangent correlation function as presented earlier in this chapter. In figure 5.16 $L_{P}$ and $\lambda$ are plotted against $d$. To facilitate a comparison with those values derived from analysis of the tangent correlation function, we plot the mean value for $L_{P}=13.1 \mu \mathrm{m}$ and the Odijk scaling law $d \propto d^{2 / 3}$ as in figures 5.10 and 5.11, respectively (dashed lines). The open red circles correspond to data sets from individual filaments whereas the closed red circles are derived from averaged data sets. Both methods of analysis - the tangent correlation functions and the radial distribution functions - lead to results which are consistent. However, the scatter in the results of analysis of the radial distribution function is larger owing to higher statistical uncertainty. Therefore, we consider the analysis of the tangent correlation function more reliable. Thus, we utilize $L_{P}=13.1 \mu \mathrm{m}$, the value which is derived from analysis of the tangent correlation function, whenever a numerical value is required in the discussions. 


\subsection{Segment Distribution in the Microchannels}

Having successfully analyzed the data in terms of tangent correlation functions and radial distribution functions, we shall now discuss a third measure to describe polymer properties. Analyzing the segment distribution of filaments near a wall sheds light on two exponents characterizing a semiflexible polymer: $\chi_{r}$ for the return probability to the wall along the polymer, that is to say, parallel to the wall, and $\theta_{r}$ for the segment distribution perpendicular to the wall.

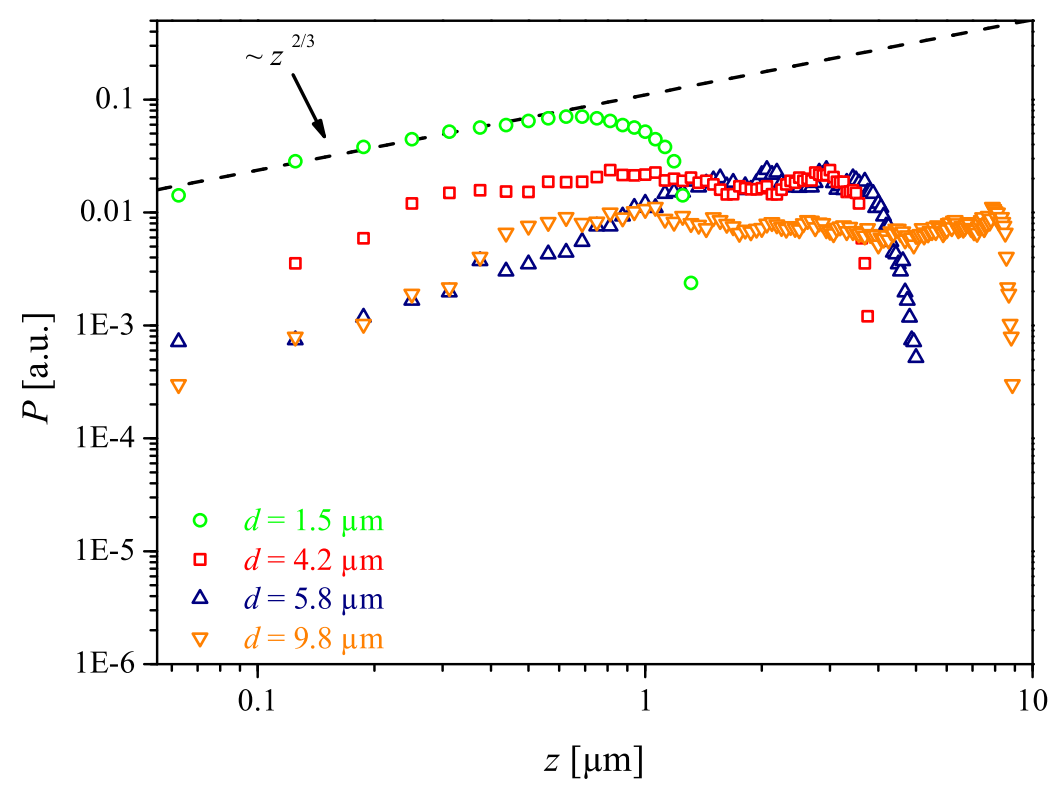

Figure 5.17: The probability $P(z)$ to find a segment of a filament in a range $[z, z+\delta z]$ plotted against $z$ on a double logarithmic scale. Symbols: data for filaments fluctuating in channels of different widths. The scaling law for the segment distribution close to the walls is confirmed (dashed line).

The segment distribution for different channel widths $(d=9.8 \mu \mathrm{m}, 5.8 \mu \mathrm{m}, 4.2 \mu \mathrm{m}$, $1.5 \mu \mathrm{m})$ is analyzed. A double logarithmic plot of the results against $z$ is shown in figure 5.17. The data are averaged over all recorded conformations of a filament and subsequently mirrored and averaged at the center line of the channel in order to improve the statistics. In this case, all curves are plotted such that the $z$-coordinate of 0 corresponds to one of the channel walls. The dashed line corresponds to the scaling law $P(z) \propto z^{2 / 3}$ (equation 4.31). Here, the improved statistics of the data for smaller $d$ are clear. Although all four data sets show the scaling, a smoother course is observed for the smaller channels.

The second exponent to be considered here, $\chi_{r}$, describes the scaling of $P(z)$ for a given $z^{*}$ in dependence of the channel width $d$. Analysis of the values for $P\left(z^{*}\right)$ 


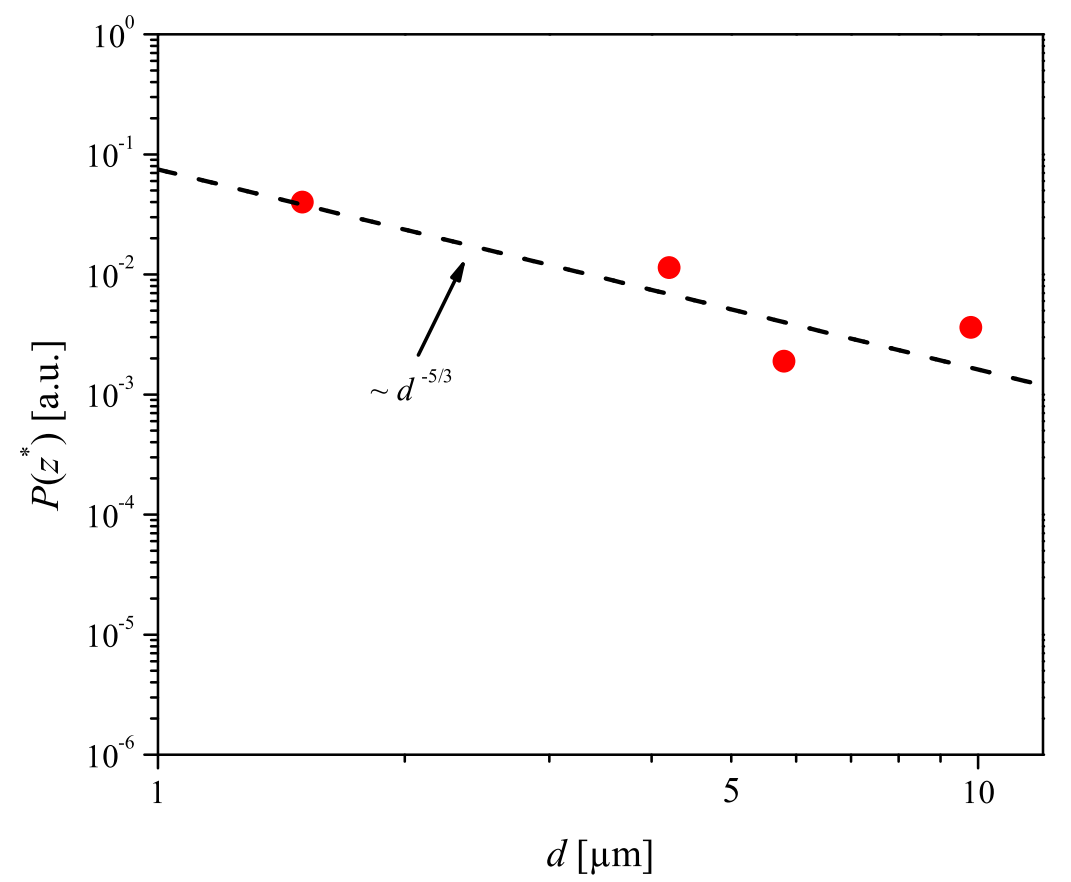

Figure 5.18: The averaged intensity $P\left(z^{*}\right)$ (first 9 data points of figure 5.17) plotted against $d$. The corresponding scaling law is confirmed (dashed line).

requires careful renormalization of the data by the mean projected length $L_{x}$ of the regarded filament. Subsequently, we average the frequencies of the first nine data points $\left(z^{*} \leq z \approx 0.6 \mu \mathrm{m}\right)$ and plot the results against $d$ (see figure 5.18 ). We choose $z \approx 0.6 \mu \mathrm{m}$ because the analysis plotted in figure 5.17 shows clearly that this is the distance from the channel wall up to which the scaling with $\theta_{r}$ holds. The dashed line in figure 5.18 denotes the appropriate scaling law for $\chi_{r}$. Since $P(z) \propto \lambda^{-\chi_{r}}$ (see equation 4.31) and $\lambda \propto d^{2 / 3}$ (see equation 4.16) we obtain with $\chi_{r}=5 / 2$

$$
P(z) \propto d^{-5 / 3}
$$

Both scaling parameters, $\theta_{r}$ and $\chi_{r}$, describe the experimental results on a length scale of $\lesssim 0.6 \mu \mathrm{m}$ in the proximity of the channel walls. By extracting the contour line from the micrographs and analyzing the statistical probability to find a filament segment at a specific distance from the wall, we can make educated assumptions about the behavior of the filaments on a length scale smaller than the optical resolution. The increase of the segment distribution at the walls with $d^{2 / 3}$ describes the depletion of segments near the wall owing to reflection of the filament by the potential. The influence of the confining wall potential on the filaments is extended quite far into the channel. Furthermore, we can comprehend that $\theta_{r}$ and $\chi_{r}$ are indeed not independent, if we consider that the probability to find the filament at 
any position $z$ within the channel is 1 and therefore the integrated probability is

$$
\int_{0}^{d} P(z) d z=1
$$

$\theta_{r}$ determines that $P(z)$ increases with $d^{2 / 3}$ at the channel walls. Together with the given channel width $d$ and the normalization condition 5.11 the absolute intensity $P(z)$ directly at the wall is a defined value. Naturally, for smaller channels $P(z)$ is a narrower distribution, whereas it is broadened for larger $d$.

\subsection{Additional Bending of Confined Filaments}

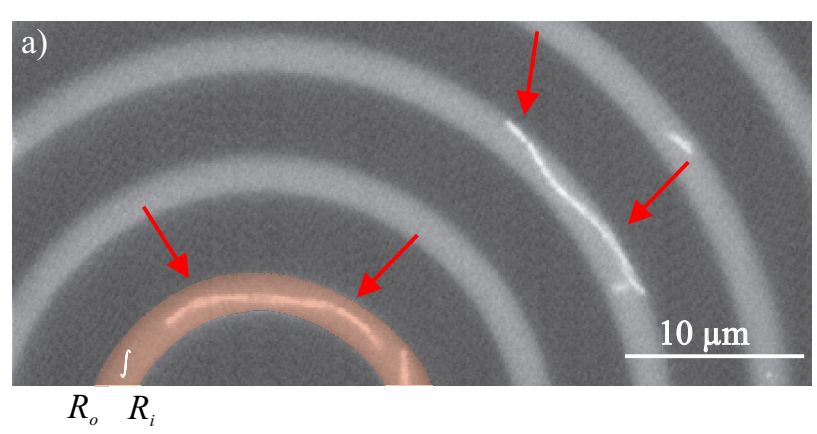

b)

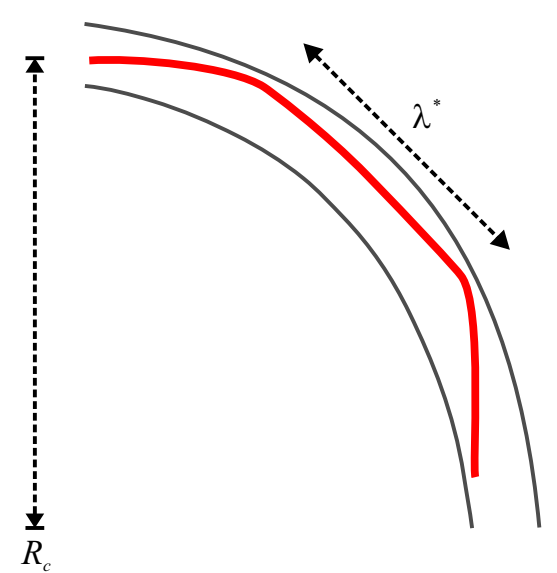

Figure 5.19: a) Overlay of a fluorescence micrograph of actin filaments in curved channels and the corresponding bright field image. b) Schematic representation of a filament fluctuating in a curved channel.

In the previous sections of this chapter we have discussed the influence of channel width and filament length on the mechanical behavior of confined semiflexible polymers. We will now present experiments where we confine actin filaments in channels with varying radius of curvature $R_{c}$ and thus investigate the influence of additional bending on the confined filaments. $R_{c}$ is defined as the radius of the center line of the channel. In figure 5.19a, an overlay of a fluorescence micrograph of actin filaments fluctuating in curved channels and the corresponding bright field image is shown. At first glance, it can be observed in the micrographs that the probability of finding a polymer is higher near the outer channel wall than at the inner channel wall. The filaments seem to avoid the additional bending by the channels by occupying the space near the outer channel wall. A schematic representation of the situation is shown in figure $5.19 \mathrm{~b}$.

In order to analyze the probability of finding a polymer at a specific position within 
a)

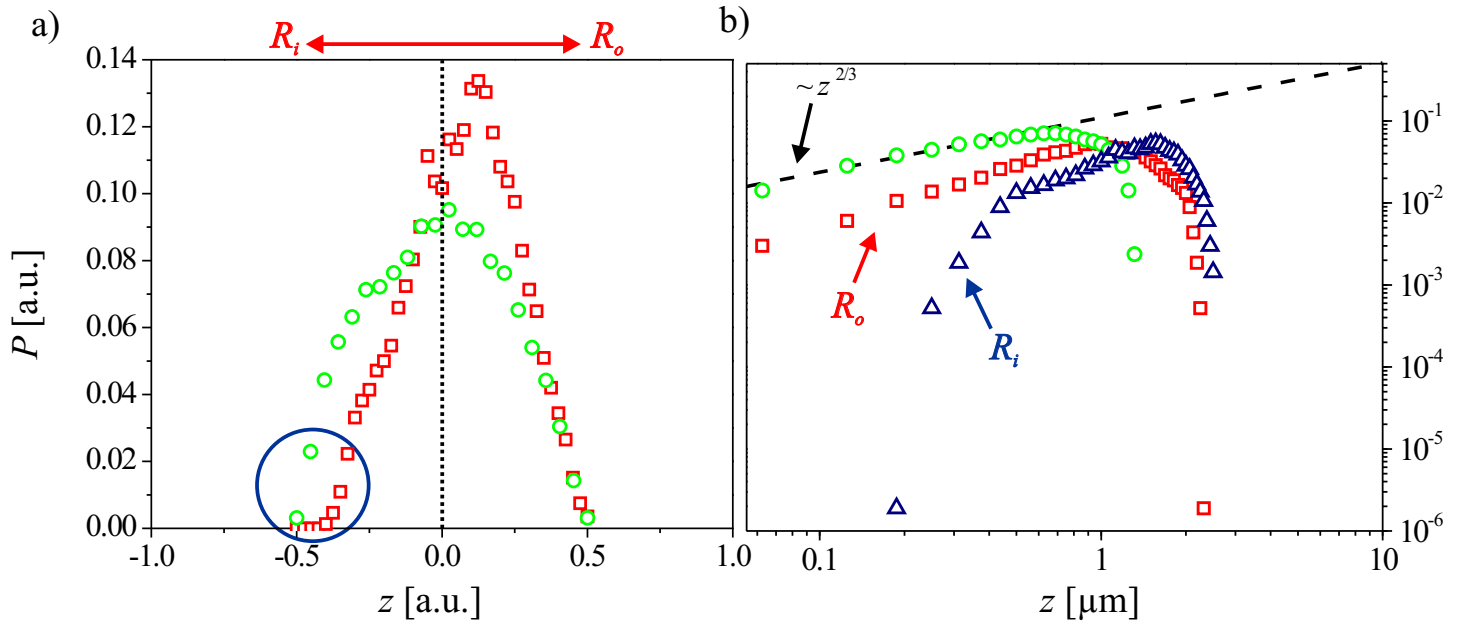

Figure 5.20: The probability $P(z)$ to find a segment of a filament at a position $z$ for a straight (green circles) and a curved (red squares, blue triangles) channel. a) $P(z)$ plotted linearly against $z$. The center line of the channel is at $z=0$. A depletion layer is found at the inner radius of curvature $R_{i}$ for the curved channels. b) Logarithmic plot of $P(z)$. Red squares: $R_{o}$ at $z=0$; blue triangles: $R_{i}$ at $z=0$; dashed line: scaling law for the segment distribution.

the channel more precisely, we determine the segment distribution in the microchannel. In this case we conduct an azimuthal integration (as indicated in figure 5.19a) along the circular channel, average the results over all recorded conformations of a filament, and plot the resulting probability $P(z)$ against the radial distance between both walls, that is to say, between the inner radius $R_{i}$ and the outer radius $R_{o}$. The resulting distribution for a filament in a channel with $d=2.5 \mu \mathrm{m}$ and $R_{c}=33.5 \mu \mathrm{m}$ is shown in figure 5.20 (red squares). We compare this result to the segment distribution of a filament in a straight channel ( $d=1.5 \mu \mathrm{m}$, green circles). In the linear plot in figure $5.20 \mathrm{a}, z=0$ is in the center of the channels. For better comparability, the channel width is normalized to 1 while keeping the area under the curve constant. Clearly, the probability distribution of the filament in the curved channel is shifted into the direction of the outer wall leaving a depletion layer in the proximity of the inner wall (indicated by the blue circle in figure 5 20 a). Such a behavior cannot be observed for straight channels, where the distribution is symmetric.

In figure $520 \mathrm{~b}$ we plot the same data on a logarithmic scale. In this case, $z=0$ is positioned at one of the channel walls. The dashed line marks the scaling law which is discussed in section 54 . For the curved channels, we analyze the behavior at both walls separately. The red squares show the segment distribution if the outer wall $\left(R_{o}\right)$ is plotted at $z=0$. The scaling of $P(z)$ comes close to the value for non-curved walls. This result is to be expected since the scaling laws derived for the segment 
a)

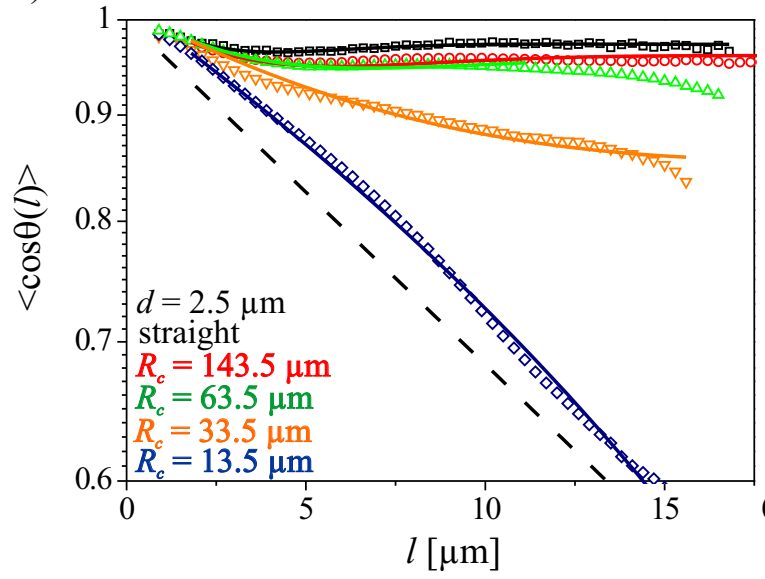

b)

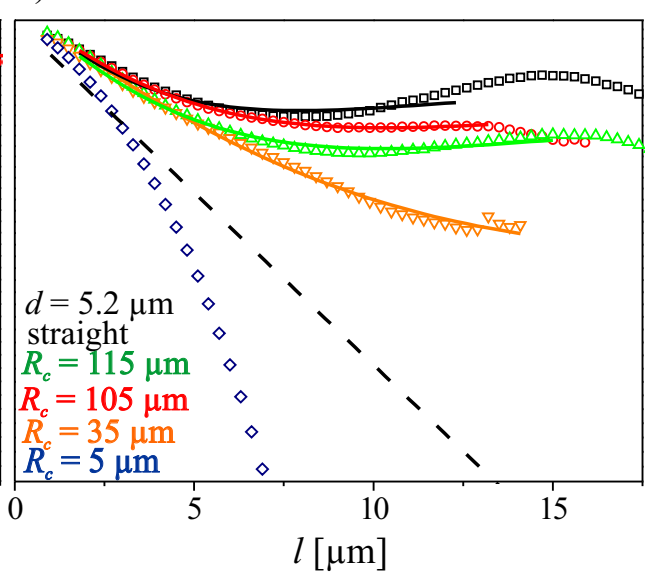

Figure 5.21: Tangent correlation functions for actin filaments fluctuating in curved channels for various radii of curvature $R_{c}$. Open symbols: experimental data; solid lines: fit curves. a) Channel width $d=2.5 \mu \mathrm{m}$; b) channel width $d=5.2 \mu \mathrm{m}$. The larger the curvature of the channels is the more the correlation is diminished as compared to a straight channel (black symbols).

distribution do not implicate a specific wall geometry. In contrast, if the inner wall $\left(R_{i}\right)$ is plotted at $z=0$ (blue triangles), the segment distribution scales differently. This is due to the depletion layer directly at the wall.

A further characterization of the filaments' mechanics is done by analyzing their contour in the curved channels. The tangent correlation functions for two different values of $d$, namely $2.5 \mu \mathrm{m}$ and $5.2 \mu \mathrm{m}$, and various values of $R_{c}$ are shown in figure 5.21 . Each curve represents data from an individual filament and the data are averaged over all recorded conformations. All filaments have a similar contour length $L \approx 20 \mu \mathrm{m}$. The radii of curvature lie in-between $5 \mu \mathrm{m}$ and $143.5 \mu \mathrm{m}$. These values are on the same order of magnitude as filament length $L$, persistence length $L_{P}$, channel width $d$, and deflection length $\lambda$. Therefore we expect an influence of the radius of curvature on the filaments' behavior. For comparison, we have included the tangent correlation function of a filament of similar contour length fluctuating in a straight channel of similar width $(1.5 \mu \mathrm{m}$ and $5.8 \mu \mathrm{m}$, respectively, black squares). The dashed lines indicate the ideal tangent correlation function of an unconfined filament $\left(L_{P}=13.1 \mu \mathrm{m}\right)$. Compared to the straight channels, the fluctuations in the curved channels are less correlated. This effect is more pronounced in channels with smaller $R_{c}$. Most filaments are yet more correlated than unconfined filaments. In order to quantify these results we assume, for a first approximation, that the situation can be described by the same formalism as filaments in straight channels. The behavior of the filaments is governed by $L_{P}$ and by deflections at the channel walls which give rise to a characteristic length scale $\lambda_{r}$. The index $r$ indicates that we dis- 


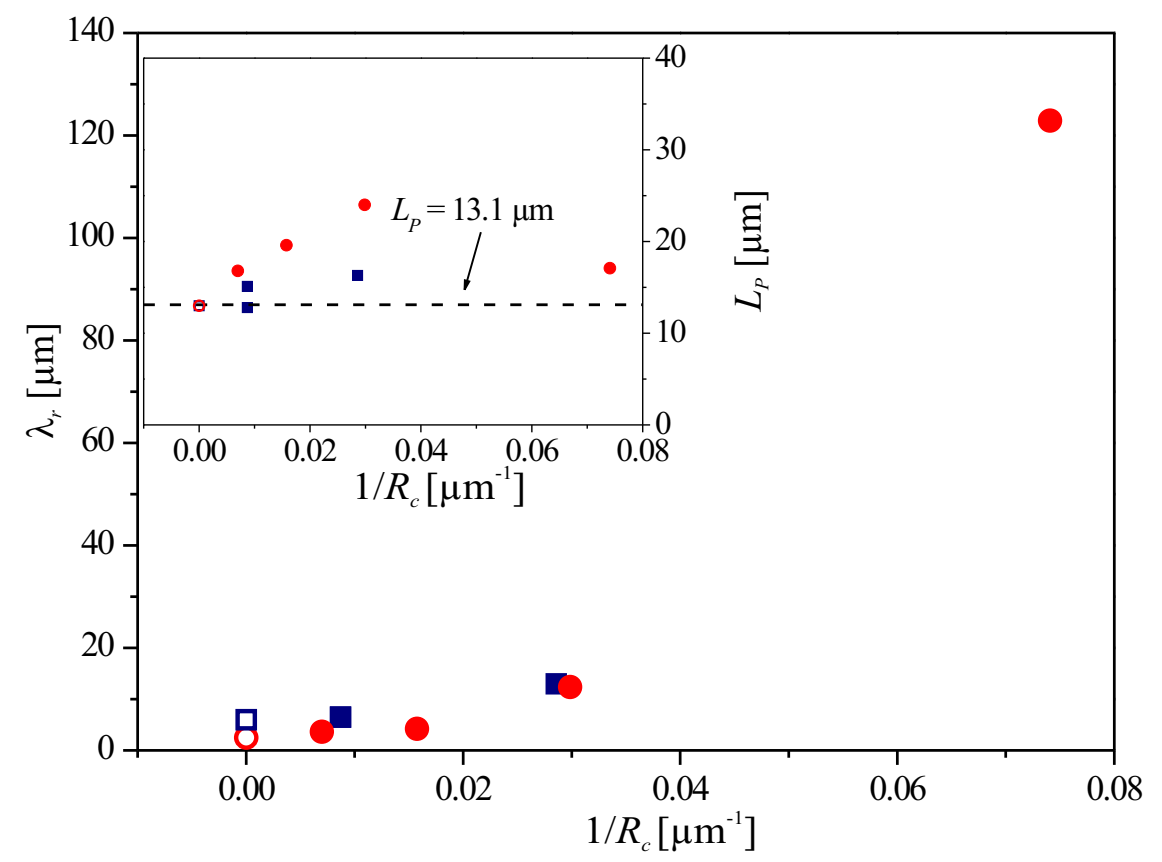

Figure 5.22: $\lambda_{r}$ plotted against the curvature $1 / R_{c}$. With increasing curvature $\lambda_{r}$ increases. Inset shows the corresponding values for $L_{P}$. Red circles: channel width $d=2.5 \mu \mathrm{m}$, blue squares: channel width $d=5.2 \mu \mathrm{m}$. The open symbols correspond to straight channels.

cuss the deflection length observed for filaments in curved channels. We fit the data curves (open symbols in figure 5.21) using equation 4.21 in consideration of the correction factor introduced in equation 5.4 as explained in section 5.1. The fit curves are shown as solid lines in figure 5.21. Only the data for the smallest $R_{c}=5 \mu \mathrm{m}$ cannot be fitted by the equations known from analysis of straight channels. This is, however, not surprising considering the fact that the curve (blue diamonds in figure $5.21 \mathrm{~b}$ ) lies even beneath the ideal tangent correlation function for unconfined filaments.

Both fit parameters, $\lambda_{r}$ (figure 5.22) and $L_{P}$ (inset), are plotted against $1 / R_{c}$. All values for $L_{P}$ lie in the expected order of magnitude. The dashed line marks the mean value from above, $L_{P}=13.1 \mu \mathrm{m} . \quad \lambda_{r}$ is increased with decreasing $R_{c}$. This result implies that the potential acting on the filaments is weaker and we therefore deal with an increased effective channel width $d_{r}$. We can depict the situation in the channels which leads to an increased deflection length $\lambda_{r}$ with decreasing radius of curvature $R_{c}$ as follows: In the case of curved channels it is possible that successive wall contacts both lie at the outer channel wall (see red arrows in figure 5.19a) as opposed to straight channels where ideally the wall contacts alternate between both walls (see for example schematic representation in figure 4.3 ). We introduce a new 
deflection length $\lambda^{*}$ which is the distance between two successive contacts at one and the same wall. The smaller the radius of curvature $R_{c}$ of the channel is the more likely the situation sketched in figure $5.19 \mathrm{~b}$ is to occur. The filament is deflected successively at the outer wall. In turn, the distance $\lambda_{r}$ between two wall contacts is, on average, larger than for weakly curved or straight channels.

Certainly, the data derived from filaments fluctuating in curved channels cannot thoroughly be described by the model developed for filaments in straight channels. The additional bending by the curved channels will have to be taken into account. However, we are able to qualitatively describe the behavior in a first estimate and obtain reasonable results.

\subsection{Future Directions}

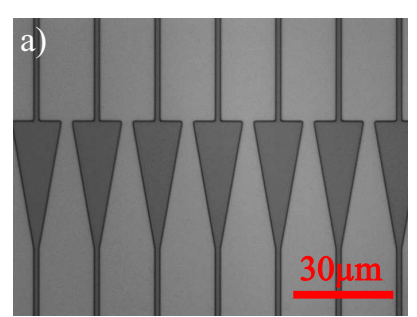

b)

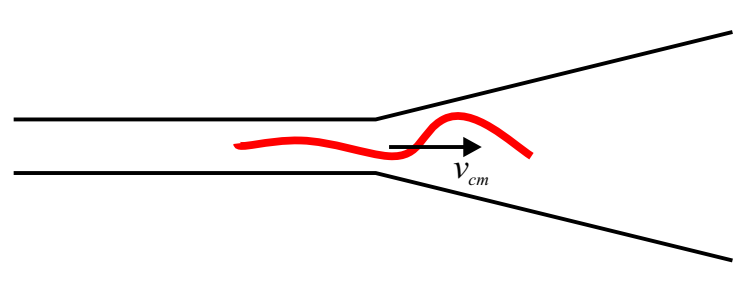

Figure 5.23: a) Micrograph of array of wedge shaped channels. b) Schematic representation of an actin filament in a wedge shaped microchannel. Owing to entropy gain it is drawn into the direction of the wider channels with a center of mass velocity $v_{c m}$.

In this chapter we have characterized individual actin filaments in an external wall potential and related the polymer fluctuations to several mechanical properties of the system. By varying filament length, channel width, and geometry, we were able to obtain information about the influence of confinement on semiflexible polymers. We showed that the behavior of confined filaments is governed by the competition of confining energy and bending energy. Furthermore, the results were successfully compared to analytical descriptions and numerical simulations of semiflexible polymers in confinement.

Future projects shall include the study of actin filaments confined in more complicated geometries, such as wedge shaped channels. In figure 5.23a, a micrograph of an array of such channels is shown. A filament could be placed with one end at the narrower part of the wedge and with the second end towards the broader end of the wedge as shown schematically in figure $5.23 \mathrm{~b}$. We expect the filament to move towards the broadest point of the wedge with center of mass velocity $v_{c m}$, 
since this is where the effective external potential is smallest. The wedges alternate with straight channel segments that have been introduced in order to detect any intrinsic flow field in the channels. Flow would, of course, interfere with the observations of the filaments' movements. However, this project likely requires the development of new flow chambers made of materials other than PDMS, such as cyclic olefin copolymers (COC), which allow for more precise control of the flow in the microchannels. Moreover, one could think of performing the polymerization of actin directly in microchannels. The assembly of actin monomers into filaments in confining environment is expected to be diffusion limited, an effect which could be further investigated by applying defined channel geometries and hydrodynamic flow fields. 


\section{Chapter 6}

\section{In Situ Collagen Self-Assembly}

Our aim in studying collagen is to gain deeper insight into the structural properties of the ECM. In section 2.2.1 the hierarchical organization of collagen in vivo has been described. In order to biomimic the system in vitro, we utilize of the wellknown fact that in bulk experiments of collagen, self-assembly is initiated by an increase in the solution $\mathrm{pH}$ from acetic to neutral or basic conditions. [61,75] It is desirable to observe fibril formation on a microscopic length scale to allow for direct visualization of the processes. The birefringent properties of collagen distinguish polarized light microscopy as an excellent method with which to carry out such studies. Additionally, we discuss X-ray microdiffraction experiments at the end of this chapter, which enable us to describe the system on the molecular length scale. Microfluidics is an optimal experimental method that provides the possibility to establish a stable $\mathrm{pH}$ gradient and at the same time is suitable for dynamic microscopy and X-ray measurements.

\subsection{Hydrodynamic Focusing and Diffusive Mixing}
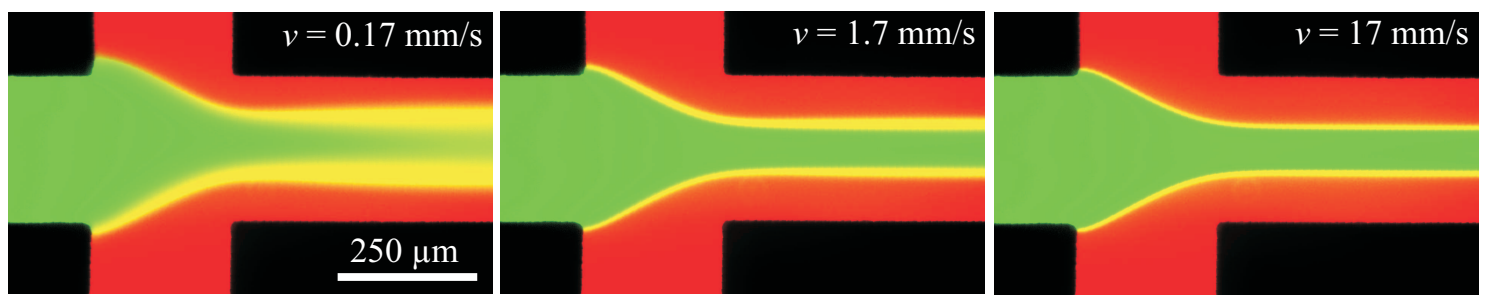

Figure 6.1: Fluorescence micrographs of the center region of crossed microchannels.

The principle of hydrodynamic focusing and diffusive mixing in laminar flow on a 
microchip has been introduced by Knight et al.[85] In a crossed channel geometry, a fluid stream that is injected into the main channel can be hydrodynamically focused by two side streams. In figure 6.1, the focused stream in the center region of such a microfluidic mixing device (see also section 3.2.2) is shown. Fluorescein solution (green) is injected into the main channel (in the figure from the left hand side), and is hydrodynamically focused by rhodamine solutions (red) injected into the side channels. The overlap regions where rhodamine and fluorescein solution are mixed by diffusion are shown in yellow. In the example shown in figure 6.1 the inlet flow velocities for the aqueous solutions in main and side channels are equal and consequently the center stream occupies a third of the full width of the outlet channel. Generally, the width of the stream can be controlled by adjusting the flow rate ratio. A faster velocity in the side channels $v_{\text {side }}$ compared to the velocity in the

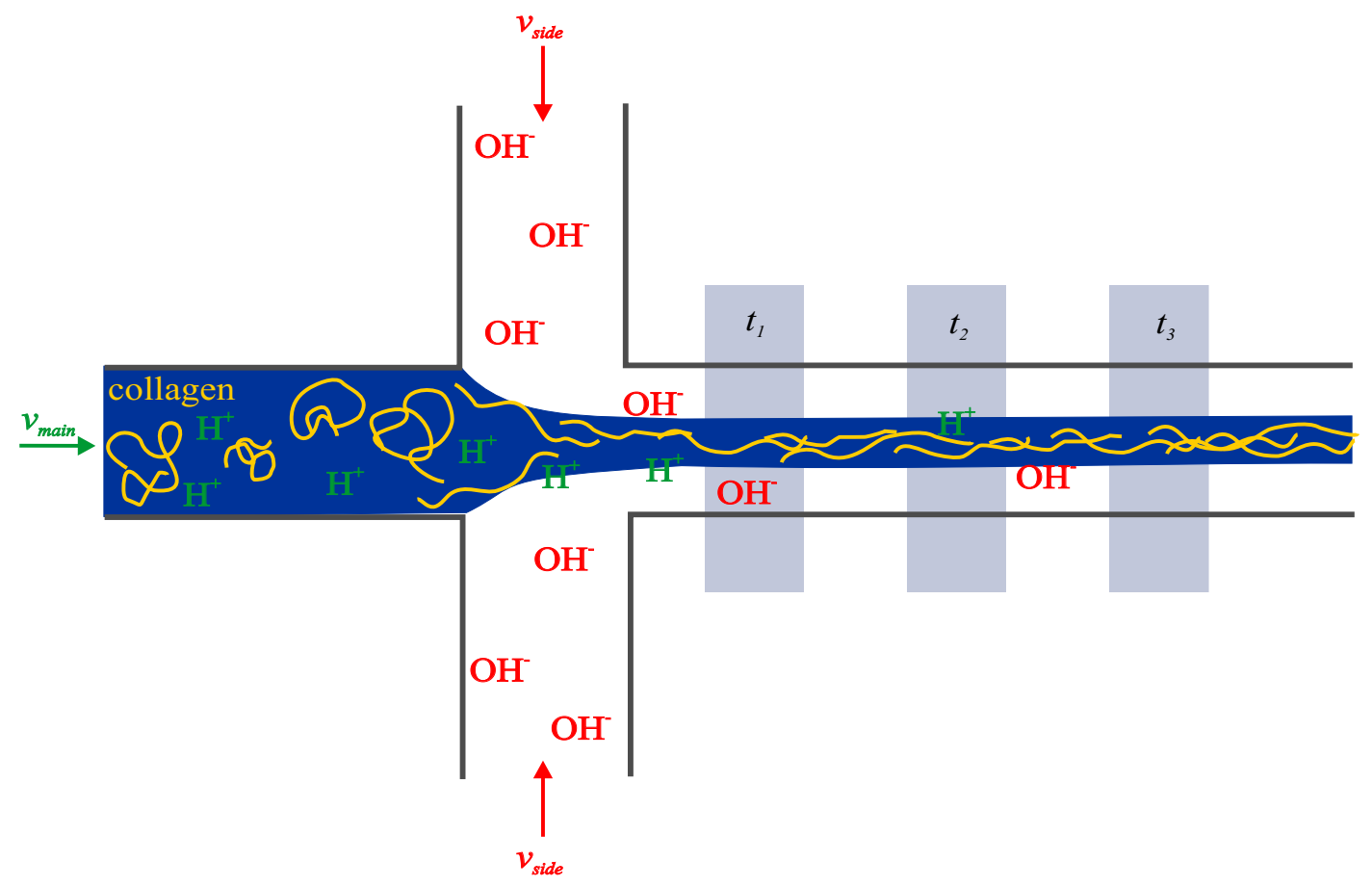

Figure 6.2: Schematic representation of the crossed channels device. The fluid in the main channel is hydrodynamically focused by the flow into the side channels. Owing to the laminar flow conditions different time points in the interaction can be observed at different positions in the outlet channel.

main channel $v_{\text {main }}$ leads to a narrower center stream. The mixing and interaction width of the components in main and side channels is decreased for higher overall velocities. This effect is obvious in figure 6.1, where the flow rates span two orders of magnitude. The higher the overall velocity is the farther extended into the outlet channel the mixing of rhodamine and fluorescein solutions is.

In figure 6.2, a schematic representation of the flow conditions in the microchan- 
nels is shown. Apart from the flow rates, diffusion constants and viscosity of the reagents in the microfluidic device determine the observable hydrodynamic and diffusive phenomena. Particularly, if the solutions in the main and side inlet channels have different $\mathrm{pH}$ values, a stable $\mathrm{pH}$-gradient is established by diffusive mixing of the solutions. This gradient enables us to perform non-equilibrium measurements in continuous flow. The sudden increase in overall flow rate at the confluence of all three inlet streams in the center of the cross additionally induces an elongational flow, which aligns and stretches the biomolecules. This allows for controlled manipulation of individual macromolecules and investigation of the influence of external stress on molecular dynamics and relaxation phenomena. Furthermore, the extension and alignment of fibrous molecules improves the formation of liquid crystalline phases. This facilitates investigation by polarized light microscopy and X-ray diffraction.

Flow can be characterized by a variety of characteristic numbers. Here, we are in particular interested in knowing whether we deal with turbulent flow which in turn would lead to time dependent flow fields. For this purpose, the dimensionless Reynolds number can be considered:

$$
R e=\frac{d \rho v}{\eta},
$$

where $d$ is a typical length scale of the system (in our case the channel width), $\rho$ is the density and $\eta$ the viscosity of the fluid, and $v$ is the flow velocity. Re characterizes the ratio of inertial and friction forces in the system; the more pronounced the former are compared to the latter, that is to say, the larger $R e$ is, the more likely it is that turbulent flow occurs. At a critical value $R e_{\text {crit }}$, which depends on the geometry of the system and is usually on the order of magnitude of 1000, the transition from laminar to turbulent flow happens. Owing to small length scales and highly viscous fluids, the highest value that is reached for any of the systems discussed here is $R e=1.6$. Thus we know that the fluids in microfluidic devices are governed by laminar flow and all fluid dynamical phenomena are stationary. Therefore, we can observe different stages of the interaction of the reagents in the center and side streams at different positions within the outlet channel (indicated by the blue shaded boxes in figure 6.2 ) because the spatial distribution of the components is temporally constant.

Altogether, the hydrodynamic focusing and diffusive mixing device represents a very well-suited method to align collagen in a controlled way while it self-assembles in a stable $\mathrm{pH}$-gradient and to observe this process in situ by means of different characterization methods. 


\subsection{Physics of the Microflow}

In order to describe the hydrodynamics of the microchannel system more precisely, the time and space dependence of flow velocities and pressure have to be understood in detail. The laminar flow can be described by the stationary Navier-Stokes equation

$$
\rho(\mathbf{v} \cdot \nabla) \mathbf{v}=-\nabla p+\eta \Delta \mathbf{v}
$$

where $\rho$ is the density of the fluid, $\mathbf{v}$ is the local flow velocity, $\nabla p$ is the pressure force per volume, and $\eta$ is the viscosity of the fluid. There is neither time dependence such as turbulence in the system nor external forces such as gravity. The left hand side of equation 6.2 is the substantive derivative of the velocity for the time independent case, whereas the right hand side contains forces caused by pressure and friction. Therefore, the Navier-Stokes equation expresses the conservation of momentum for fluid systems. Furthermore, our system is free of divergence, which ensures conservation of mass,

$$
\nabla \mathbf{v}=0
$$

Moreover, diffusive phenomena have to be included. More precisely, the diffusion of collagen molecules, $\mathrm{AcH}$, and $\mathrm{NaOH}$ has to be considered. Fick's first law,

$$
\mathbf{j}=-D \nabla c
$$

indicates that the diffusion flux is proportional to the concentration gradient $\nabla c$ with a coefficient that is the diffusion constant $D$. In combination with the continuity equation,

$$
\frac{\partial c}{\partial t}=-\nabla \mathbf{j}
$$

which states that the concentration can only change upon a net flux at the considered position, we obtain Fick's second law, also known as the diffusion equation:

$$
\frac{\partial c}{\partial t}=D \Delta c
$$

The description is complicated by the fact that equations $6.2,6.3$, and 6.6 depend on one another and have to be coupled in order to solve the problem thoroughly. Examples for diffusion constants are $D_{\text {collagen }}=6.9 \cdot 10^{-12} \mathrm{~m}^{2} / \mathrm{s}, D_{\mathrm{OH}^{-}}=5.3 \cdot 10^{-9} \mathrm{~m}^{2} / \mathrm{s}$, and $D_{A c H}=1.24 \cdot 10^{-9} \mathrm{~m}^{2} / \mathrm{s}$ in water.[112]

In our case, the diffusion of $\mathrm{NaOH}$ into the collagen stream in the center of the flow does not occur in aqueous solution but rather in polymer solution. Obviously, the presence of long chain molecules influences the diffusion of small-sized molecules 


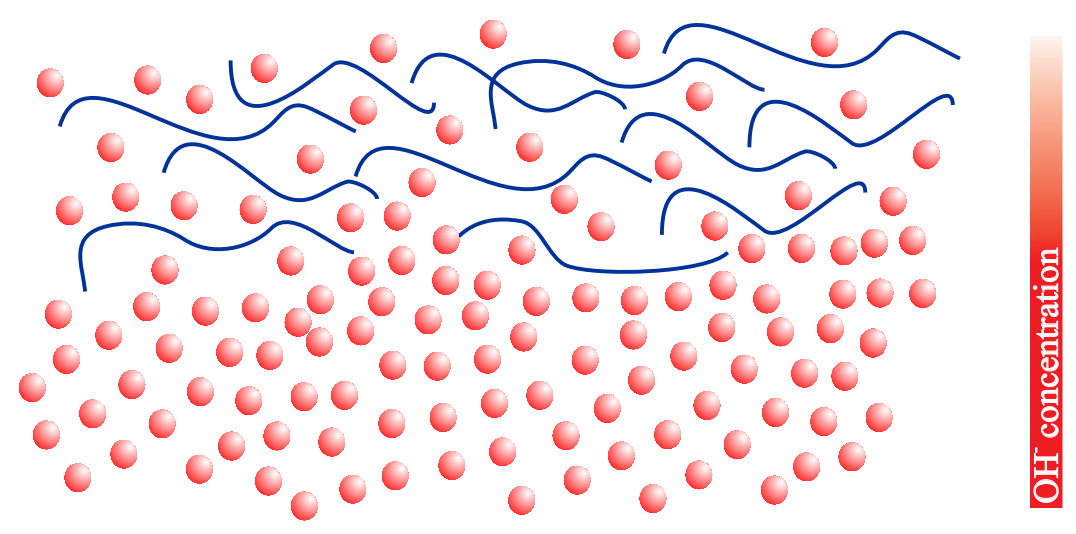

Figure 6.3: Schematic representation of the diffusion of small molecules $\left(\mathrm{OH}^{-}\right.$in our case) in a semidilute polymer solution. The diffusion path length is increased.

and solvents in these polymer solutions or gels as schematically shown in figure 6.3. The diffusive behavior is strongly governed by the concentration of the polymer solution. For flexible polymers such as collagen, three different regimes can be distinguished.[96] (i) In dilute solutions the polymers are separated from each other, do not interact, and each molecule occupies a spherical region corresponding to the radius of gyration $R_{g}$. (ii) In semidilute solutions the molecules start to overlap. (iii) In concentrated solutions the polymers are strongly entangled and contour fluctuations become small. Here, we shall discuss the transition from the dilute to the semidilute regime for collagen solutions at a concentration $c=10 \mathrm{mg} / \mathrm{mL}$. The contour length of a collagen molecule is $L=295 \mathrm{~nm}$ and the persistence length is $L_{P}=14.5 \mathrm{~nm}$. [113] If we approximate the collagen molecules as ideal chains, we obtain $R_{g}=L \cdot L_{P} / 3 \approx 38 \mathrm{~nm}$. The critical concentration $c^{*}$ at the boundary of the semidilute regime is defined by

$$
\frac{c^{*} N_{A}}{M_{W}} \frac{4}{3} \pi R_{g}^{3} \simeq 1
$$

where $M_{W}$ is the molecular weight and $N_{A}$ is Avogadro's number.[96] The relation 6.7 can be understood by considering that at $c^{*}$ the number of molecules in a unit volume is $c^{*} N_{A} / M_{W}$. For collagen molecules we obtain $c^{*} \approx 2 \mathrm{mg} / \mathrm{mL}$. Therefore a solution with $c=10 \mathrm{mg} / \mathrm{mL}$ is to be classified as semidilute.

Solvent diffusion in semidilute polymer solutions can be described by the concept of obstruction effects. The fundamental principle in the corresponding models is that the polymer chains are regarded as motionless relative to the diffusing molecules (solvents or solutes). [114] This approximation can be justified because the selfdiffusion coefficient of polymers is orders of magnitudes smaller than that of the diffusing solvent molecules. The existence of polymers in the solution increases the path length of the diffusant molecules because they have to navigate through the 
polymer landscape. In the Maxwell-Fricke model the effective diffusion constant $D_{\text {eff }}$ is given by

$$
D_{e f f}=\frac{1-\phi}{1+\phi / \chi} D
$$

where $\phi$ is the volume fraction of the polymer plus non-diffusing solvent bound to it and $\chi$ is a form factor, for example $\chi=1.5$ for rods.[115] A collagen solution of $c=10 \mathrm{mg} / \mathrm{mL}$ corresponds to $\phi=0.01$; we obtain $D_{\text {eff }}=0.98 D$. A stronger decay of $D_{\text {eff }}$ with the polymer concentration is considered in the model of Mackie and Meares [116], where

$$
D_{e f f}=\left(\frac{1-\phi}{1+\phi}\right)^{2} D
$$

still yields $D_{\text {eff }}=0.96 D$. These estimates show that it is well justified to set $D_{\text {eff }} \approx D$ in the case of a volume fraction of $\phi=0.01$. Realistically, experiments with collagen solutions cannot be conducted with much higher concentrations owing to the limited solubility of the protein. Larger aggregates of collagen molecules, which result from self-assembly, can also be described by this model insofar as they are treated as thicker "polymers". However, it is important to note that although semidilute polymer solutions in general are highly viscous, the Stokes-Einstein equation, which inversely relates viscosity $\eta$ and diffusion constant $D,[112]$ is not valid. The Stokes-Einstein relation applies to fluids that are continuously viscous also on a molecular length scale. In the case of polymer solutions the macroscopic appearance is that of a viscous fluid, but on a microscopic scale it is highly discontinuous.

\subsection{The Finite Element Method}

Like many other partial differential equations, the stationary Navier-Stokes equation and the diffusion equation can indeed be analytically solved for simple geometries and problems but not for more complex, discontinuous systems, where additionally several equations have to be coupled, like the one we address here. In this case, numerical approximations are more suitable. The finite element method (FEM) is one of the most widely used and powerful techniques to numerically solve partial differential equations having given boundary conditions. Basically, the considered area is divided into a finite number of subareas (the finite elements) having a simple shape (for example triangles or tetrahedrons) and the physical problem is formulated on each of the subareas. The global problem is thus transformed into solvable, local problems. The solution is carried out by defining so-called ansatz functions whose linear combinations provide possible solutions for the numerical approximation. The 
physical problem is solved separately on each of the subareas while considering the adjacent subareas. This leads to a large linear system of equations which can in many cases only be solved using a computer.

We use the commercial software Femlab to solve the problem of diffusive mixing of sodium hydroxide $(\mathrm{NaOH})$ and acetic acid $(\mathrm{AcH})$ in the presence of highly viscous collagen solution in two dimensions using about 10,000 finite elements. For this purpose we couple the stationary incompressible Navier-Stokes equation and the respective diffusion equations for each component, namely collagen, $\mathrm{NaOH}$ and AcH. Owing to the complex situation and coupling of several physical phenomena the simulation of the real three-dimensional case is not possible with the available computing power. However, it can be shown that even though the ratio of channel width $d$ and depth $h$ indeed influences the flow profiles, the principle physical phenomena are not affected.[117]

\subsection{Hydrodynamics in Microchannels}

\subsubsection{Polarized Light Microscopy}

Collagen is highly birefringent, a trait shared among many fibrous proteins. The signal is increased by (i) the alignment and assembly of many individual collagen molecules into liquid crystalline structures and (ii) anisotropic elongation and extension. [118] Polarized light microscopy has been widely used to study collagen
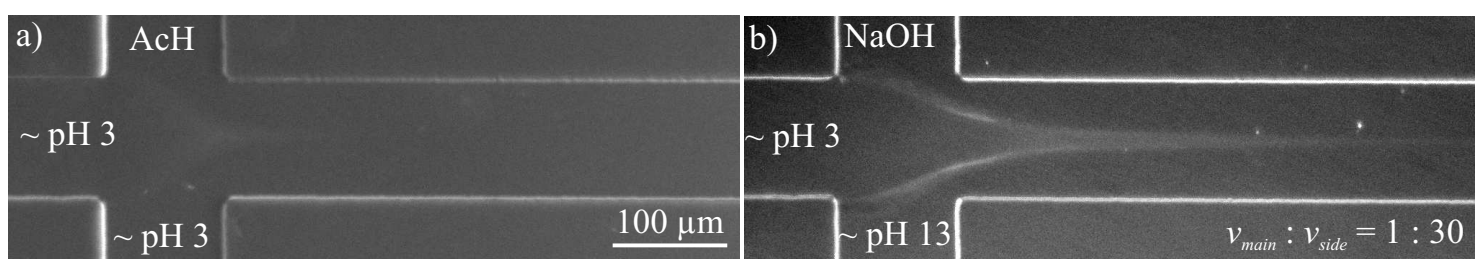

Figure 6.4: Birefringence micrographs of collagen streams hydrodynamically focused by a) $\mathrm{AcH}(\sim \mathrm{pH} 3), \mathrm{b}) \mathrm{NaOH}(\sim \mathrm{pH} 13)$.

from the early 19th century on.[119-121] In figure 6.4 birefringence micrographs of hydrodynamically focused collagen streams are shown. The flow rate ratio is $v_{\text {main }}: v_{\text {side }}=1: 30$, and the absolute flow rates are $v_{\text {main }}=1.35 \mathrm{~mm} / \mathrm{s}$ and $v_{\text {side }}=40.5 \mathrm{~mm} / \mathrm{s}$. If the collagen (at a concentration of $10 \mathrm{mg} / \mathrm{mL}$ ) is diffusively mixed with its solvent $(0.075 \mathrm{M} \mathrm{AcH}, \sim \mathrm{pH} 3)$ flowing into the side inlet channels, the $\mathrm{pH}$ in the system is constant in all channels. Thus, the collagen remains in its soluble form and no self-assembly occurs. Consequently, the observable birefringence 
signal is weak (figure 6.4a). On the contrary, if the AcH is replaced by a strongly basic solution of $\mathrm{NaOH}(0.075 \mathrm{M}, \sim \mathrm{pH} 13)$ a stable $\mathrm{pH}$ gradient is established in the outlet channel. The collagen begins to self-assemble, and we observe a stronger birefringence signal at the edges of the collagen stream (figure 6.4 b). Therefore the regions within the stream where the collagen is assembled can be identified by an increase of the birefringence signal.

\subsubsection{Influence of Solution Viscosity}

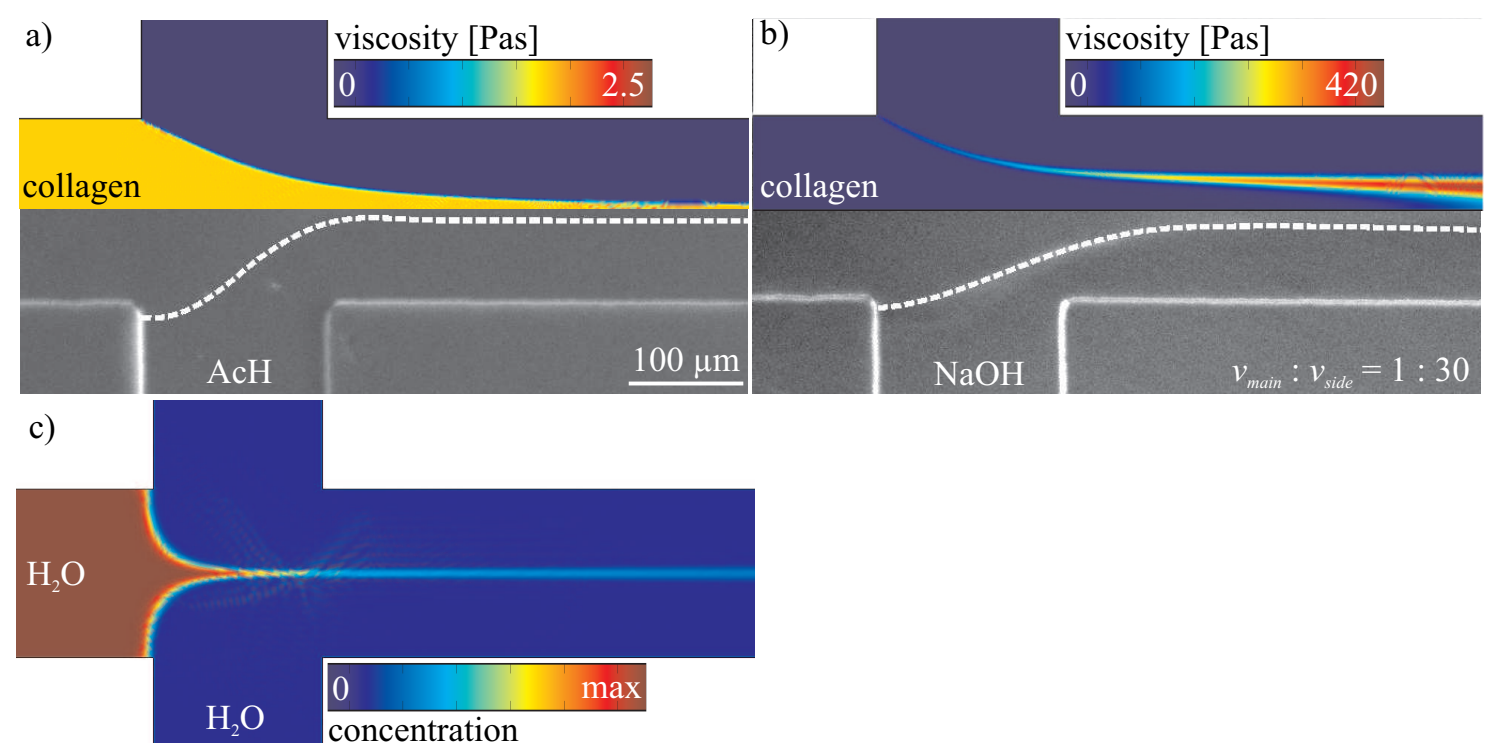

Figure 6.5: Simulation of the viscosity in the collagen stream when hydrodynamically focused by a) $\mathrm{AcH}(\sim \mathrm{pH} 3)$ and b) $\mathrm{NaOH}(\sim \mathrm{pH} 13)$. Model and experiment agree well. Note that both scales for viscosity differ by two orders of magnitude. c) For comparison: simulated concentration distribution of a hydrodynamically focused stream in a water/water system.

In addition to the increase of the birefringence signal upon self-assembly of the collagen molecules, a clear change in shape of the collagen stream can be observed. From reference experiments and FEM simulations we know that such a change in shape is caused by an increase in the solution viscosity. Indeed, bulk experiments have shown that the viscosity of a collagen solution is dramatically increased once the $\mathrm{pH}$ is increased and the collagen gelation process advances. [122] As the $\mathrm{pH}$ increases and consequently the collagen self-assembly proceeds, the form of the focused collagen stream in the crossed channel device changes. Namely, it becomes more extended into the outlet channel. In order to illustrate this point, figure $65 \mathrm{sc}$ shows the simulated concentration distribution of aqueous solution without collagen $(\eta=0.891 \mathrm{mPa} \cdot \mathrm{s})$ injected into the main channel. Thus, we have aqueous solutions 
in all three inlet channels and regard the simulated shape of the center stream. Apart from the viscosity, all parameters such as channel geometry and flow rates are the same. The center stream is extremely narrow: as expected, it occupies a fraction of $\sim 1 / 60$ of the outlet channel, corresponding to the relative flow rates $\left(v_{\text {main }}: v_{\text {side }}=1: 30\right)$.

We perform FEM simulations in order to quantify the influence of viscosity effects and understand the hydrodynamics of the system. First, we consider the system with spatially constant $\mathrm{pH}$ as is shown in figure $6.4 \mathrm{a}$. In this case the viscosity in the center stream corresponds to the value for soluble collagen at a concentration of $10 \mathrm{mg} / \mathrm{mL}$. In order to obtain a comparative value we use an Ubbelohde-viscometer to conduct bulk measurements and the result is $\eta_{\text {collagen }} \approx 2 \mathrm{~Pa} \cdot \mathrm{s}$. This value is more than 2000 times higher than the viscosity of water $\left(\eta_{\text {water }}=0.891 \mathrm{mPa} \cdot \mathrm{s}\right)$, which is the main component of the solvent (acetic acid, $0.075 \mathrm{M}$ ). The result is particularly impressive when it is regarded that the volume fraction of collagen is merely about $\phi=0.01$. The FEM simulations include the Navier-Stokes equation and the diffusion equations for three inflowing streams where the center stream has a much higher viscosity than the side streams. The viscosity is fitted to $\eta_{\text {collagen }}=2000 \eta_{\text {water }}=1782 \mathrm{mPa} \cdot \mathrm{s}$ which is in agreement with the result from bulk measurements. $\eta_{\text {collagen }}$ is assumed to be proportional to the cube of $c_{\text {collagen }}$ thus slightly smoothing the transition in viscosity between the center and side streams. This approximation is used to accomplish better numerical handling of the system. The simulated viscosity in direct comparison to the experimental results is shown in figure $6.5 \mathrm{a}$. Generally, in the experiment the center stream is slightly bulged into the side channels; this feature is not reflected in the simulations. We assume that this feature is caused by interfacial tension and wetting effects at the interface of both miscible solutions. Apart from this detail, experiment and simulation agree well and the shape of the collagen stream can be fitted. The results demonstrate the consistency of bulk viscometry, microfluidics experiments, and FEM simulations. In turn, this technique provides a method which enables us to evaluate the viscosity of minute quantities of very viscous fluids.

To characterize the collagen assembly, we consider the system where the $\mathrm{pH}$ gradually changes within the microchannel (figure 6.4b). We aim to numerically describe the following situation: $\mathrm{NaOH}$ diffuses into the center stream, which changes the $\mathrm{pH}$ of the collagen solution and induces self-assembly of the collagen molecules which, in turn, leads to an increase of the viscosity. Therefore, we have to couple the viscosity to the local $\mathrm{pH}$ of the system which is a function of the solvent concentrations $c_{\mathrm{NaOH}}$ and $c_{A c H}$. The functional dependence of the viscosity on the $\mathrm{pH}$ and thus on the 

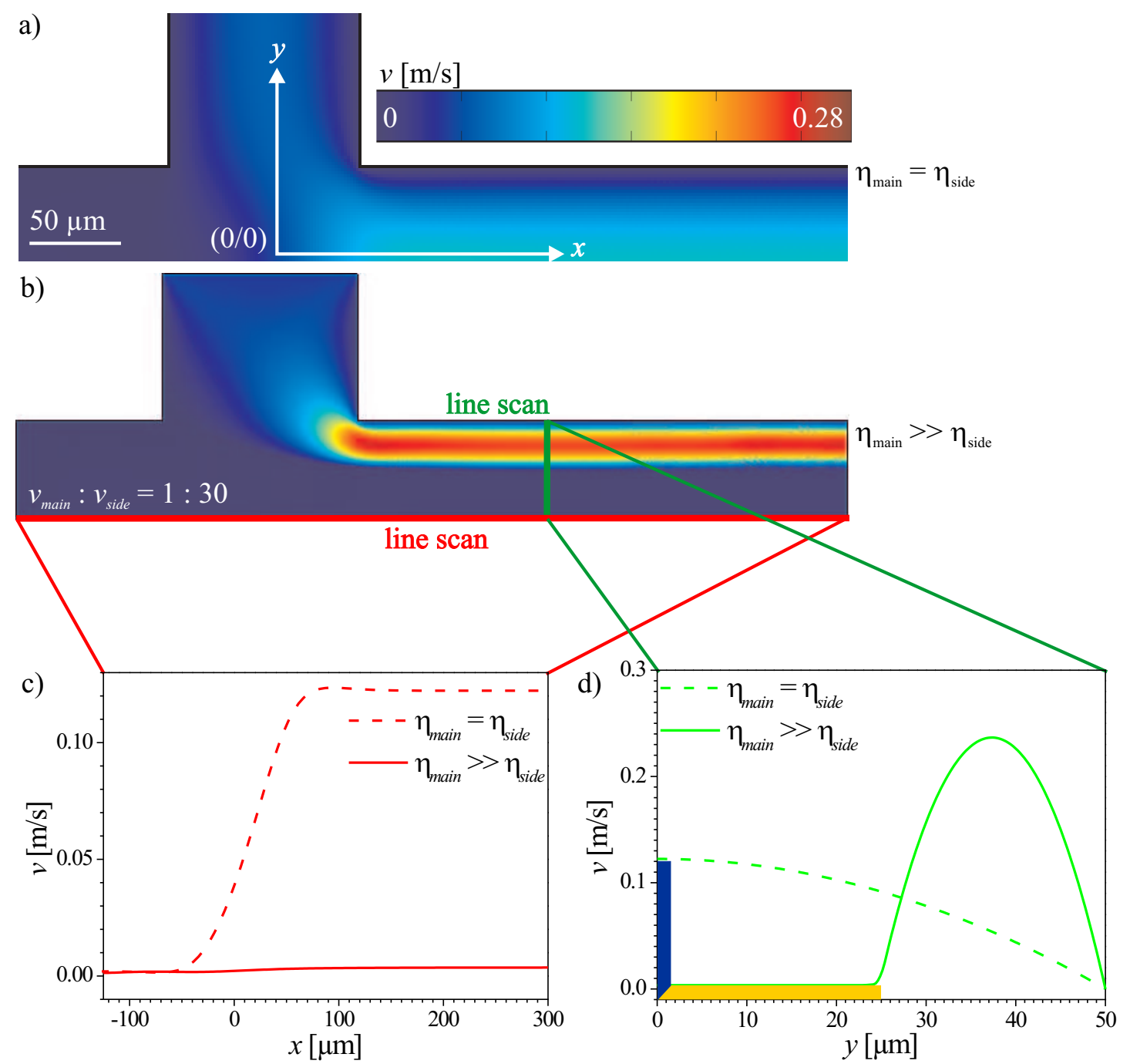

Figure 6.6: Simulation of the velocity field in the microfluidic device. The absolute flow rates are $v_{\text {main }}=1.35 \mathrm{~mm} / \mathrm{s}$ and $v_{\text {side }}=40.5 \mathrm{~mm} / \mathrm{s}$. a) For a water $/$ water system $\left(\eta_{\text {main }}=\eta_{\text {side }}=0.891 \mathrm{mPa} \cdot \mathrm{s}\right)$. b) For self-assembling, highly viscous collagen in the main channel $\left(\eta_{\text {main }} \gg \eta_{\text {side }}\right)$. c) Line scans of the velocity field in $x$-direction (compare red line in b). The origin of the coordinate system is in the center of the cross. d) Line scans of the velocity field in $y$-direction (compare green line in b). The region occupied by the center stream is indicated by the blue (water/water system) and yellow (collagen system) boxes. 
acid and base concentrations has to be described by a single function. The situation can be well approximated by

$$
\eta=\eta_{\text {water }}+\eta_{\text {collagen }}+\eta_{\text {assembled }}
$$

with

$$
\eta_{\text {assembled }}=r_{1} c_{\text {collagen }}^{3}\left(\tanh \left(r_{2}\left(c_{O H^{-}}-c_{A c H}\right)\right)+1\right),
$$

with two fit parameters $r_{1}$ and $r_{2}$. The numerical value of $2 r_{1}$ determines the maximum viscosity of the assembled collagen and $r_{2}$ determines the shape of the function. $\eta_{\text {collagen }}=1782 \mathrm{mPa} \cdot \mathrm{s}$ is known from the control experiment without collagen assembly. The relation 6.11 is chosen such that the point of inflection is at the stoichiometric point, that is to say, where the concentrations of $\mathrm{AcH}$ and $\mathrm{NaOH}$ are equal. We obtain good results for $r_{1}=245 \mathrm{~Pa} \cdot \mathrm{s}$ and $r_{2}=100$ as shown in figure 6.5b. Note that the scale for the viscosity differs from the one in figure 6.5 a by two orders of magnitude. The viscosity of the assembled collagen $\eta_{\text {assembled }}$ is extremely high, five orders of magnitude higher than $\eta_{\text {water }}$. These results agree remarkably well with bulk experiments. For gelated collagen solutions with $c_{\text {collagen }}=3-5 \mathrm{mg} / \mathrm{mL}$ a viscosity of $\eta_{\text {collagen }} \approx 140 \mathrm{~Pa} \cdot \mathrm{s}$ has been determined.[122] Our results are on the same order of magnitude and slightly higher due to the higher collagen concentration. The microfluidics experiments in combination with FEM simulations provide a method to analyze the complete process of collagen assembly in terms of solution viscosity. This is especially noteworthy because the numerical values of the viscosities in the system span five orders of magnitude and are coupled via the neutralization reaction. The results using microfluidics are in agreement with bulk measurements while - owing to laminar flow - providing the additional possibility to investigate the process in a time resolved manner.

In the following section the influence of the viscosity on the flow fields in the microchannels shall be discussed. Figures $6.6 \mathrm{a}$ and b show the simulated flow fields for a water/water system $\left(\eta_{\text {main }}=\eta_{\text {side }}=0.891 \mathrm{mPa} \cdot \mathrm{s}\right)$ and, in direct comparison, the collagen system which we study here $\left(\eta_{\text {main }} \gg \eta_{\text {side }}\right.$, compare also figure $6.4 \mathrm{~b}$ and $6.5 \mathrm{~b}$ ). Both simulations are scaled equally to facilitate the comparison. Obviously, an increased viscosity in the center stream leads to a dramatically increased flow rate of the side stream fluid at the confluence of all inflows, whereas the flow rate of the center stream itself remains almost constant. This is in striking contrast to the water/water system. In figure $6.6 \mathrm{c}$, line scans along the center of the main channel parallel to the flow direction are shown (see red line in figure $6.6 \mathrm{~b}$ ). For the water/water system the flow is strongly accelerated in the center of the cross, where immediately three times the fluid volume as before flows through the channel. Since 
all inflowing streams have the same viscosity, they behave like one single flow. This can also be observed in a line scan perpendicular to the main channel direction as shown in figure 6.6 d for $x=150 \mu \mathrm{m}$. Along the $y$-direction, the typical parabolic flow profile is developed. Since $v_{\text {main }} \ll v_{\text {side }}$, only a very small fraction of the main channel is occupied by the center stream as indicated by the blue box in figure 6.6 d. In contrast to this anticipated behavior, the collagen system behaves strikingly differently. Owing to its high viscosity, the flow velocity of the center stream is not as influenced by the inflowing side streams. Instead, the side streams are strongly accelerated. Consequently, the velocity profile parallel to the flow direction in the center of the main channel only very slowly increases and the acceleration region, where added biomolecules can be elongated, is extended into the main channel (figure 6.6c). Perpendicular to the flow direction, we observe parabolic flow profiles developing in both side streams whereas the flow velocity throughout the center stream remains constant in the lateral direction. The interfaces between collagen solution and side streams act like additional "walls" and the side streams flow as if in channels of smaller width. Eventually, the expected parabolic flow profile throughout the whole channel evolves. However, this occurs far downstream and is therefore not accounted for in our discussions. Fortunately, we can actually take advantage of the hydrodynamic conditions in the crossed channels. The slower but longer-lasting acceleration phase is used to align the collagen molecules in a more defined way.

\subsection{Collagen in $\mathrm{pH}$ Gradient}

\subsubsection{Diffusive Phenomena}

The FEM simulations also give detailed information about the concentration distribution of each reagent in the channels as shown in figure 6.7 for a flow rate ratio of $v_{\text {main }}: v_{\text {side }}=1: 30$ and $c_{N a O H}=0.075 \mathrm{M}$. The simulated collagen distribution (a) yields a constant concentration $(10 \mathrm{mg} / \mathrm{mL})$ throughout the entire collagen stream and does not noticeably diffuse out of the center stream. This is due to the fact that the diffusion of collagen molecules in aqueous solution $\left(D_{\text {collagen }}=6.9 \cdot 10^{-12}[112]\right)$ is much slower than the diffusion of the relatively smaller $\mathrm{OH}^{-}$ions and $\mathrm{AcH}$ molecules. For numerical reasons, we consider a diffusion constant which is higher $\left(D_{\text {simulation }}=8 \cdot 10^{-11}\right)$, but it is still low enough to reflect the real conditions quite well. The $\mathrm{AcH}$ (b) is more localized than the $\mathrm{NaOH}$ (c), which diffuses very fast into the center stream. We can understand these differences on the following two grounds. First of all, the diffusion constant of $\mathrm{OH}^{-}\left(D_{O H^{-}}=5.3 \cdot 10^{-9} \mathrm{~m}^{2} / \mathrm{s}\right)$ is three 
a)

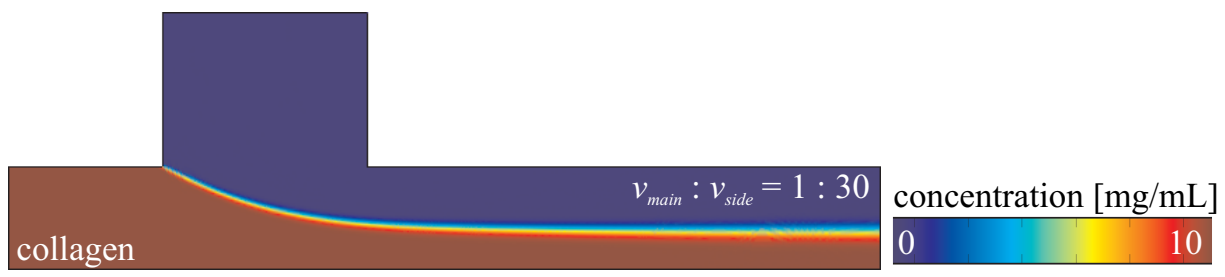

b)

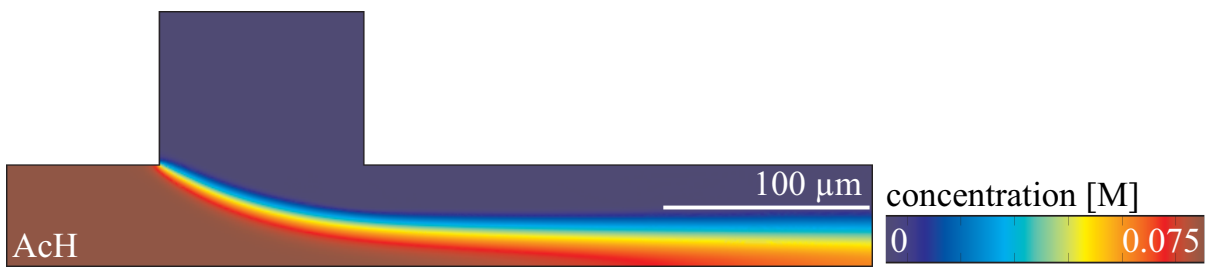

c)

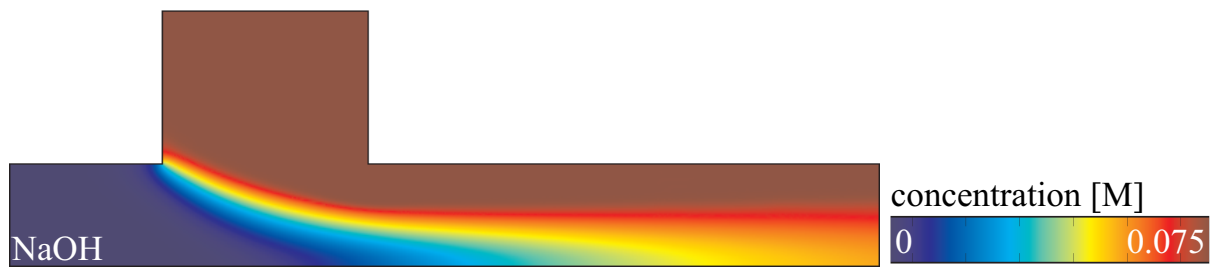

Figure 6.7: Simulated concentration distribution of a) collagen, b) $\mathrm{AcH}$, and c) $\mathrm{NaOH}$ within the microchannels. Collagen is strongly localized whereas $\mathrm{AcH}$ and $\mathrm{NaOH}$ diffuse faster.

times larger than of $\mathrm{AcH}\left(D_{A c H}=1.24 \cdot 10^{-9} \mathrm{~m}^{2} / \mathrm{s}\right)$. Secondly, AcH diffuses out of the center stream and is flushed away by the suddenly increasing flow in the side streams (see discussion in section 6.4.2) before it has the opportunity to diffuse out further. Note that here we treat collagen and AcH solutions separately, since their diffusive behavior differs even though they are injected into the main channel as a mixture. Corresponding concentration distributions can be determined for different flow rate ratios and give information about the local acid/base mixture at every position of the microfluidic device.

\subsubsection{Calculation of the $\mathrm{pH}$}

The concentration profiles from the FEM simulation are used to calculate the local $\mathrm{pH}$ distribution. In our system, collagen is dissolved in $0.075 \mathrm{M} \mathrm{AcH}$ and diffusively mixed with $0.075 \mathrm{M}-1 \mathrm{M} \mathrm{NaOH}$. For this reason we will discuss the problem here using these numbers. However, the equations can be adjusted for any titration of a weak acid with a strong base. The following definitions and abbreviations are used in the calculation and discussion (all concentrations are functions of the position in the device):

$$
\begin{array}{ll}
K_{s} & \text { acidity constant }\left(1.78 \cdot 10^{-5} \text { for } \mathrm{AcH}\right) \\
K_{w} & \text { dissociation constant of water }\left(1 \cdot 10^{-14}\right)
\end{array}
$$


$\left[\mathrm{H}^{+}\right] \quad$ hydronium ion concentration

$\left[\mathrm{OH}^{-}\right] \quad$ total hydroxide concentration, which consists of input hydroxide (corresponds to $\left[\mathrm{Na}^{+}\right]$) and additionally forming hydroxide from dissociation of water and the formation of $\mathrm{AcH}$

$\left[\mathrm{Na}^{+}\right] \quad$ available concentration of sodium ions, corresponds to local "input" $\mathrm{NaOH}$

$\left[\mathrm{Ac}^{-}\right] \quad$ acetate concentration

$[\mathrm{AcH}]_{\text {free }}$ free (not dissociated) $\mathrm{AcH}$

$[\mathrm{AcH}]_{\text {input }}$ local "input" $\mathrm{AcH}$ concentration

As discussed in section 6.5.1, we obtain the local concentrations of $\mathrm{NaOH}$ and $\mathrm{AcH}$ from FEM simulations. In the following section we will develop a method to calculate the $\mathrm{pH}$ as a function of the channel position in dependence of these concentration distributions. [123] For this purpose we define two more parameters, $e=$ $[\mathrm{AcH}]_{\text {input }}$, which is the $\mathrm{AcH}$ concentration as determined by FEM simulations, and $n=\left[\mathrm{Na}^{+}\right] / e$. The sodium ion concentration corresponds to the added hydroxide concentration. Since there is additional hydroxide present in the system we use the sodium ion concentration as a measure of the input hydroxide concentration as determined by FEM simulations.

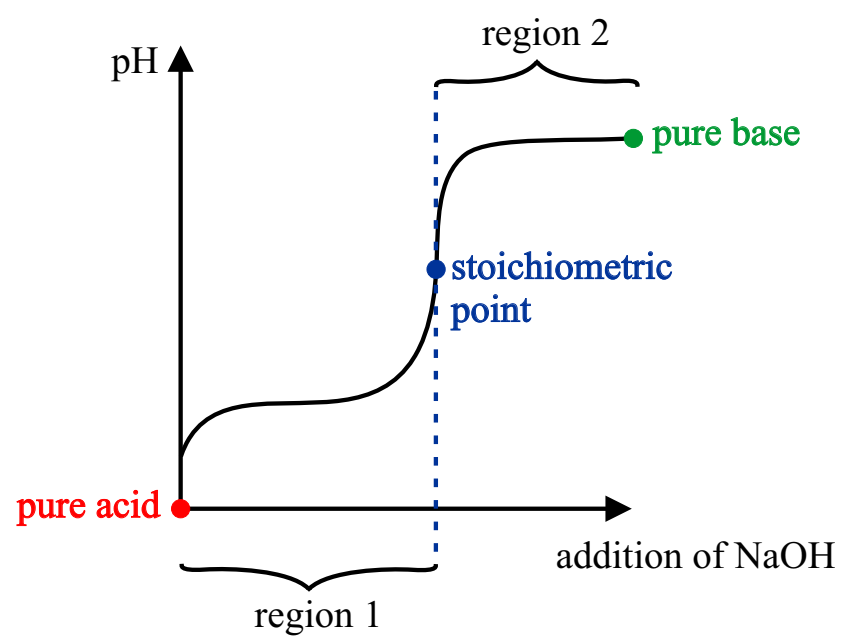

Figure 6.8: Titration curve of a weak acid with a strong base.

In principle, a pH titration of a weak acid $(\mathrm{AcH})$ with a strong base $(\mathrm{NaOH})$ is conducted in the microfluidic device. However, the situation is more complicated since both the concentration of $\mathrm{AcH}$ and $\mathrm{NaOH}$ change constantly. A titration curve such as the one shown in figure 6.8 is only valid for one specific $\mathrm{AcH}$ concentration. It follows that the $\mathrm{pH}$ for the addition of different volumes of $\mathrm{NaOH}$ can be determined 
accordingly. In our case, because the AcH concentration also changes spatially, we have, in a way, to determine such a titration curve for every single AcH concentration.

An analytical expression exists for a few very specified points on a titration curve, such as for pure weak acid (AcH in our case), that is, $n=0$

$$
p H=\frac{1}{2}\left(p K_{s}-\log _{10}\left([\mathrm{AcH}]_{\text {input }}\right)\right),
$$

pure strong base (NaOH in our case), that is, $e=0$

$$
p H=p K_{w}+\log _{10}\left(\left[\mathrm{Na}^{+}\right]\right),
$$

and the stoichiometric point, where the molarity of the weak acid equals the molarity of the strong base, that is, $n=1$

$$
p H=\frac{1}{2}\left(p K_{s}+p K_{w}+\log _{10}\left(\frac{1}{2}[\mathrm{AcH}]_{\text {input }}\right)\right) .
$$

For the remaining points on the titration curve before the stoichiometric point is reached the $\mathrm{pH}$ is usually approximated by the Henderson-Hasselbalch equation and, even more inconveniently, there is no such approximation available after the stoichiometric point is reached. In order to find an analytical expression for the whole titration curve we determine the equations which define the problem and solve this system of equations using Mathematica.

\section{The System of Equations}

It is necessary to perform a case differentiation and to set up a system of equations for the conditions before and after the stoichiometric point is reached, respectively. Before the stoichiometric point is reached we begin with pure acid solution, so we have to consider the definition of the acidity constant

$$
K_{s}=\frac{\left[\mathrm{H}^{+}\right]\left[\mathrm{Ac}^{-}\right]}{[\mathrm{AcH}]_{\text {free }}}
$$

and the definition of dissociation constant of water

$$
K_{w}=\left[\mathrm{H}^{+}\right]\left[\mathrm{OH}^{-}\right]
$$

When the AcH dissociates, the total number of acetate ions needs to remain constant. The input AcH dissociates partially to acetate and partially remains in the system as free $\mathrm{AcH}$ :

$$
[\mathrm{AcH}]_{\text {input }}=[\mathrm{AcH}]_{\text {free }}+\left[\mathrm{Ac}^{-}\right]
$$


Upon addition of $\mathrm{NaOH}$ these equilibrium conditions are affected. We need to ensure that the number of charges is conserved:

$$
\left[\mathrm{H}^{+}\right]+\left[\mathrm{Na}^{+}\right]=\left[\mathrm{Ac}^{-}\right]+\left[\mathrm{OH}^{-}\right]
$$

Equations 6.15 - 6.18 have to be coupled and solved in order to determine the $\mathrm{pH}$ before the stoichiometric point is reached as a function of the acid and base concentrations.

After the stoichiometric point is reached, we must take into account that a sufficient amount of $\mathrm{NaOH}$ has now been added to the system in order to dissociate all input $\mathrm{AcH}$ (this is, in fact, the definition of the stoichiometric point). However, the resulting acetate ions react with water molecules, which in turn leads to additional formation of $\mathrm{OH}^{-}$according to

$$
\mathrm{H}_{2} \mathrm{O}+\mathrm{Ac}^{-} \rightarrow \mathrm{OH}^{-}+\mathrm{AcH}
$$

Therefore, the effective $\mathrm{OH}^{-}$concentration in the system stems from the input $\mathrm{OH}^{-}$ (corresponds to $\left[\mathrm{Na}^{+}\right]$), diminished by the $\mathrm{OH}^{-}$which dissociates the input $\mathrm{AcH}$ and additionally the newly formed $\mathrm{OH}^{-}$(equation 6.19 , corresponds to $[\mathrm{AcH}]_{\text {free }}$ ). This leads to the following relation:

$$
\left[\mathrm{OH}^{-}\right]=[\mathrm{AcH}]_{\text {free }}+\left[\mathrm{Na}^{+}\right]-[\mathrm{AcH}]_{\text {input }} .
$$

Equations $6.15-6.18$ and 6.20 need to be coupled and solved.

\section{Solution of the System of Equations}

The equations can be solved analytically using Mathematica. For $0<n<1$, that is, before the stoichiometric point is reached (region 1 in figure 6.8), we obtain the following solution:

$$
p H=-\log _{10}\left[\frac{\frac{1}{3}\left(-K_{s}-e n\right)+2^{1 / 3} \xi}{3\left(\zeta+\sqrt{4 \xi^{3}+\zeta^{2}}\right)^{1 / 3}}-\frac{1}{3 \cdot 2^{1 / 3}}\left(\zeta+\sqrt{4 \xi^{3}+\zeta^{2}}\right)^{1 / 3}\right],
$$

where

$$
\xi=-K_{s}^{2}-3 K_{s} e+K_{s} e n-3 K_{w}-e^{2} n^{2}
$$

and

$$
\zeta=2 K_{s}^{3}-18 K_{s} K_{w}+9 K_{s}^{2} e-\left(3 K_{s}^{2}-9 K_{w}\right) e n+9 K_{s} e^{2} n-3 K_{s} e^{2} n^{2}+2 e^{3} n^{3} .
$$


For $n>1$, that is, after the stoichiometric point is reached (region 2 in figure 6.8), the solution is

$$
p H=-\log _{10}\left[\frac{-\delta+\sqrt{4 e n K_{s} K_{w}+\delta^{2}}}{2 e n}\right]
$$

where

$$
\delta=e K_{s}+K_{w}-e n K_{s}
$$

At first glance, the results 6.21 and 6.24 seem to be very complicated; however, both equations only depend on two parameters: $e$, which denotes the $\mathrm{AcH}$ concentration and $n$, which corresponds to the $\mathrm{NaOH}$ concentration, both of which are determined by the FEM simulations. The equations are implemented in a Matlab program in order to calculate the $\mathrm{pH}$ at every specific position in the considered microfluidic device.

\subsubsection{Comparison of Experiment and Simulation}

Calculating the $\mathrm{pH}$ distribution in the crossed channels yields the possibility to directly compare experiment and simulation and thus determine how collagen selfassembly is related to the local pH. In figure 6.9, birefringence micrographs of the collagen assembly process at three different flow rate ratios are shown. The respective concentration distributions of $\mathrm{AcH}$ and $\mathrm{NaOH}$ together with equations 6.21 and 6.24, which describe the local $\mathrm{pH}$ in dependence of these concentrations, are used to calculate the complete $\mathrm{pH}$ conditions for every position in the microchannels. Since the reaction kinetics are faster than the diffusion, we assume that self-assembly takes place immediately after the solutions are mixed and the $\mathrm{pH}$ has reached a certain threshold value. The shape of the birefringence signal and the shape of the simulated $\mathrm{pH}$ distribution correspond well for all three flow rate ratios. A strong increase in birefringence intensity, which is presumably due to self-assembly and consequently lateral arrangement of collagen molecules, can be observed in the regions where $\sim \mathrm{pH} 6-7$ (green). Interestingly, this $\mathrm{pH}$ value has been associated with the onset of the assembly of collagen I for bulk experiments.[124] This result shows that our experimental findings as well as the numerical description are consistent.

The birefringence micrographs as well as the simulated data are further compared by analyzing line scans at specific positions in the channel (figure 6.10). For each line scan of the birefringence intensity, the signal is averaged over 15 images and 20 lines in each image and the resulting intensity is normalized. The points of maximum intensity correspond to the borders of the flow where the collagen is already assembled. In figure 6.10a three different positions $(x=50,100,200 \mu \mathrm{m})$ in the 

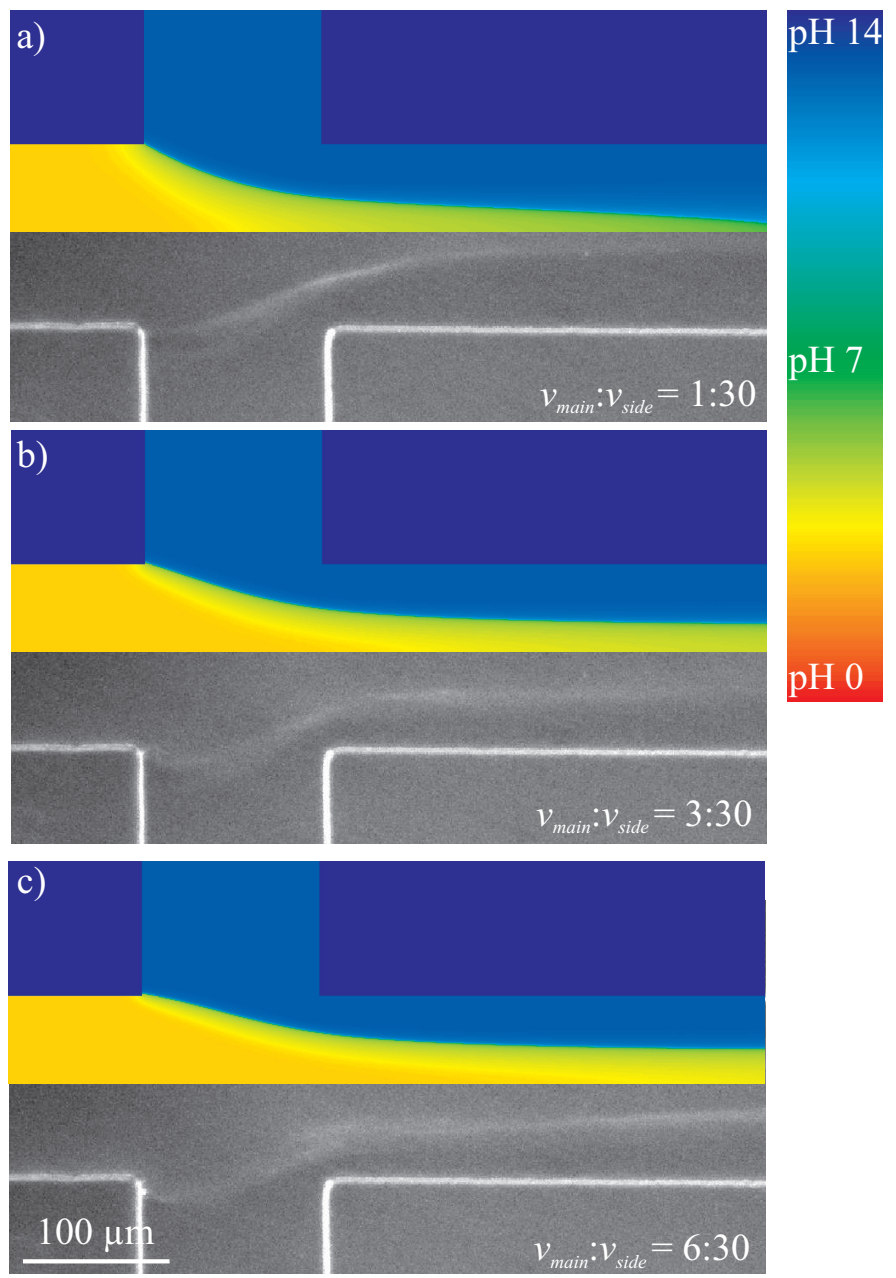

Figure 6.9: Birefringence micrographs of the collagen solutions at different flow rate ratios and corresponding FEM simulations of the $\mathrm{pH}$ distribution within the crossed channels.

crossed channels are marked by colored lines. The corresponding line scans for a fixed flow rate ratio $v_{\text {main }}: v_{\text {side }}=3: 30$ are shown in figure 6.10b. The solid lines show the birefringence data and the dashed lines the corresponding course of the $\mathrm{pH}$ distribution. Each pair of curves intersects at approximately $\mathrm{pH} 7$ which supports our observation that the self-assembly occurs at a neutral $\mathrm{pH}$. The fact that we find agreement for all analyzed positions emphasizes the validity of the assumptions we made for the simulations.

In figure $6.10^{\circ}$ line scans of the $\mathrm{pH}$ distribution along the main channel direction in the center of the channel are given. The $\mathrm{pH}$ does not increase to neutral in the considered region and, as expected in the light of this fact, we do not observe a strong increase of the birefringence intensity along the main channel. In figure f.10d we compare line scans at the same position $x=100 \mu \mathrm{m}$ in the channel for different flow rate ratios. Again, the solid lines show the birefringence intensity whereas the 

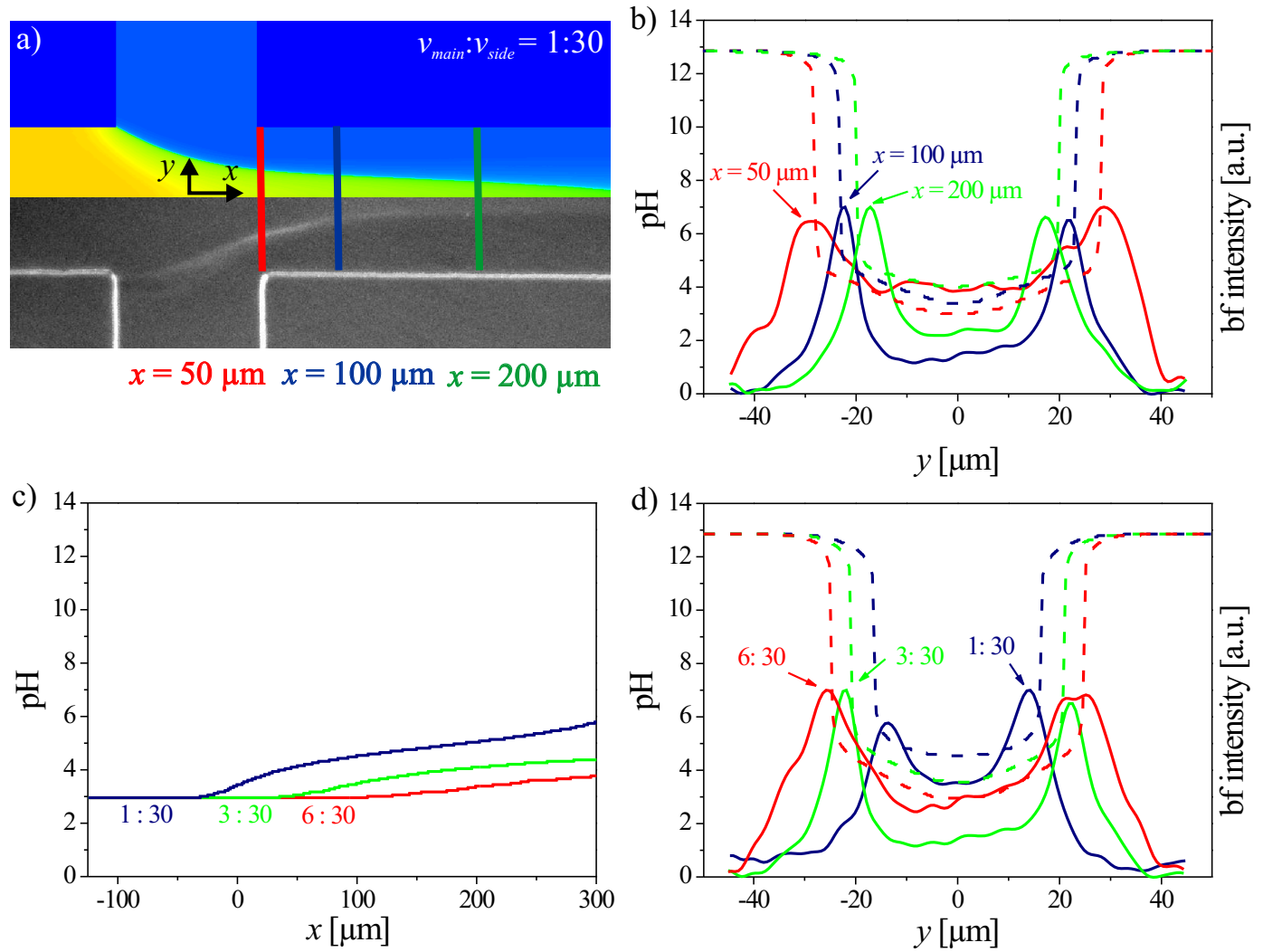

Figure 6.10: a) Positions for the line scans of $\mathrm{pH}$ and birefringence intensity shown in $\mathrm{b}$. The origin $(x, y)=(0,0)$ is defined as the center of the cross. b) Line scans perpendicular to the main channel direction at different positions in the channel for a flow rate ratio $v_{\text {main }}: v_{\text {side }}=3: 30$. Solid lines: birefringence signal; dashed lines: $\mathrm{pH}$ distribution. c) Line scans of the simulated $\mathrm{pH}$ distribution parallel to the main channel direction in the center of the channel for different flow rate ratios. d) Line scans perpendicular to the main channel direction at $x=100 \mu \mathrm{m}$ for different flow rate ratios. Solid lines: birefringence signal; dashed lines: $\mathrm{pH}$ distribution.

dashed lines show the corresponding $\mathrm{pH}$ distribution. In this case we observe a better agreement of experiment and simulation for slower side stream velocities.

Altogether, however, we find that the experimental system can be well described by the FEM simulations and the determination of the $\mathrm{pH}$ distribution in the channels is well suited to understand the processes of fibril formation. The diffusive mixing of $\mathrm{NaOH}$ and acidic collagen solution leads to a stable $\mathrm{pH}$ gradient, which in turn induces self-assembly of the collagen molecules starting at a $\mathrm{pH}$ of $\sim 5-7$. The self-assembly can directly be visualized by an increase in birefringence signal and coincides with a dramatic increase in solution viscosity from $\sim 2 \mathrm{~Pa} \cdot \mathrm{s}$ up to $\sim 500 \mathrm{~Pa} \cdot \mathrm{s}$. At the same time, we are able to model the conditions in the microchannels. This allows for semi-quantitative determination of the interdependence of viscosity, $\mathrm{pH}$ distribution, and flow profiles. Our results, which agree well with 
corresponding bulk measurements, show that the technique is indeed suitable to investigate reacting non-equilibrium systems on the microscale.

\subsection{X-Ray Microdiffraction}

a)

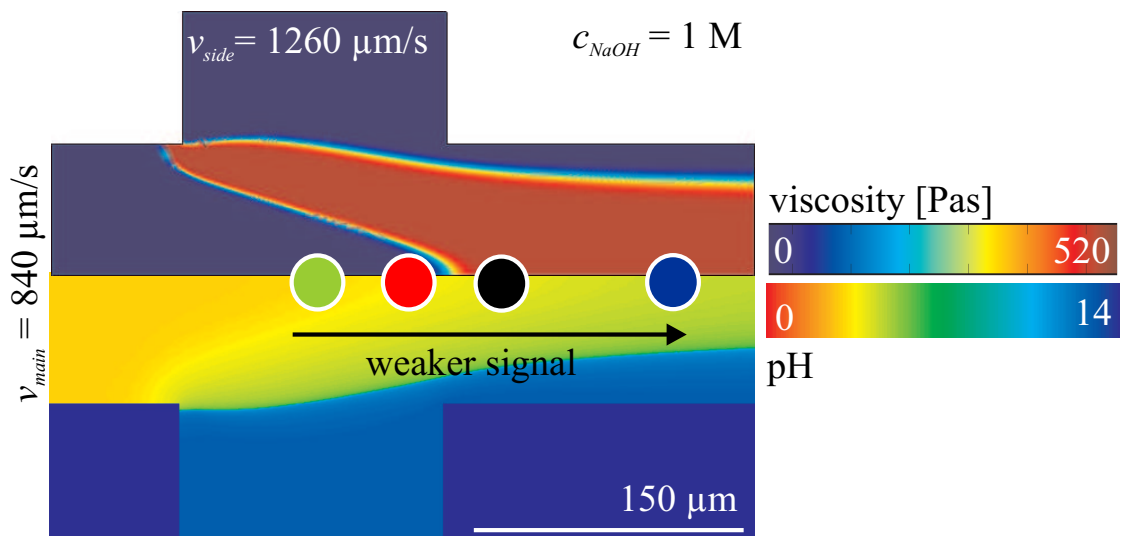

b)

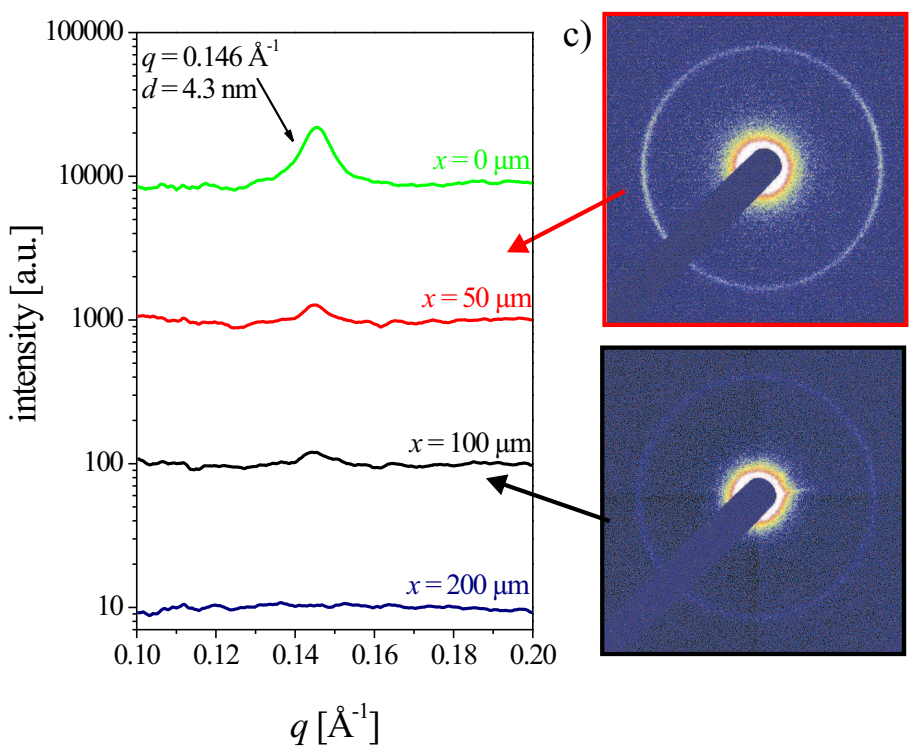

Figure 6.11: Results of the X-ray microdiffraction measurements at different positions along the center stream. a) Simulation, top: viscosity; bottom: $\mathrm{pH}$ distribution. The circles indicate the measuring positions. b) Series of X-ray scans measured at the positions marked in a. c) Diffraction patterns for two different measuring positions.

In section 6.5 we have presented results of polarized light microscopy experiments and FEM simulations that established the study of collagen self-assembly and characterization of the system on a micrometer scale. However, it is also highly desirable to study the system on a molecular length scale. At the same time, it is our aim to investigate the dynamics of fibril formation. Therefore, X-ray microdiffraction in combination with microfluidics is an excellent tool for our purposes. A unique 
a)

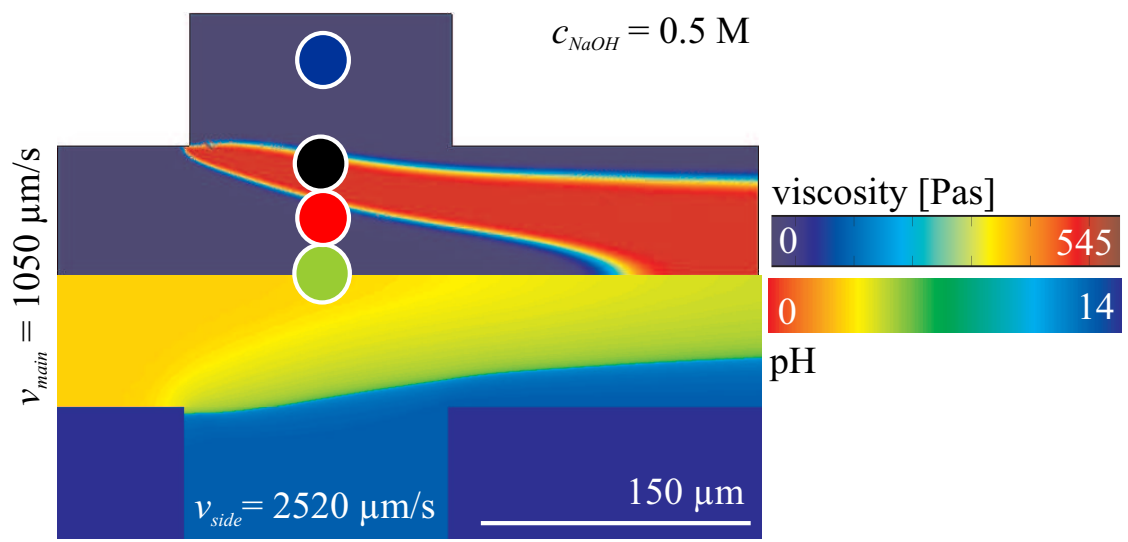

b)

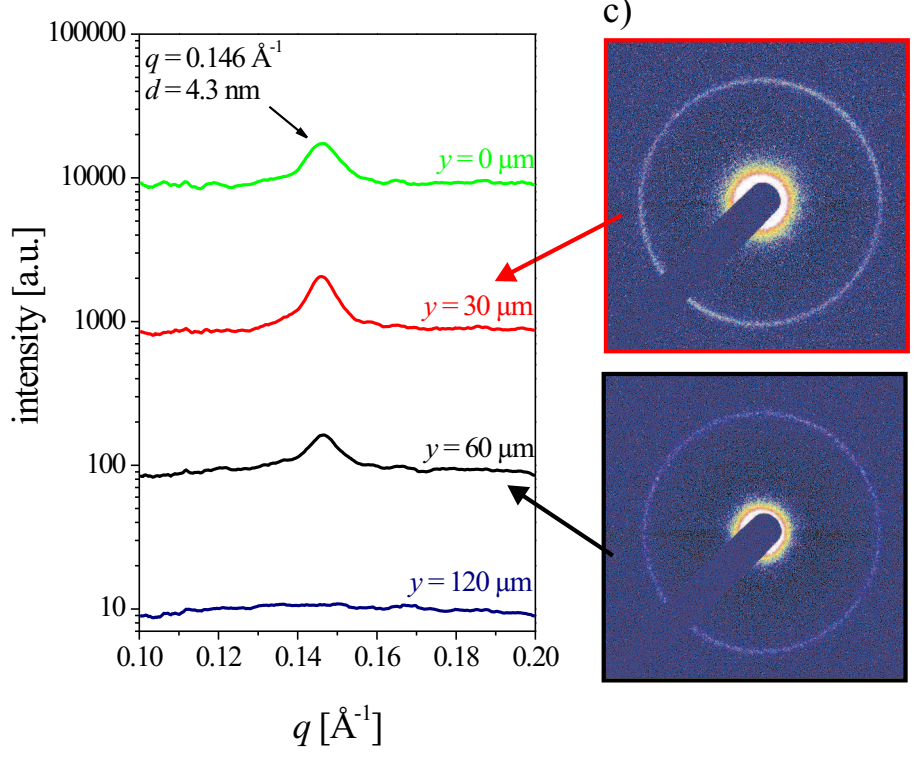

Figure 6.12: Results of the X-ray microdiffraction measurements at different positions perpendicular to the center stream. a) Simulation, top: viscosity; bottom: $\mathrm{pH}$ distribution. The circles indicate the measuring positions. b) Series of X-ray scans measured at the positions marked in a. c) Diffraction patterns for two different measuring positions.

feature of our setup is that the steady, continuous flow enables in situ investigation of the pH-induced collagen assembly without material damage. In combination with a microfocused X-ray beam we are particularly well-qualified to investigate the molecular packing and ordering of collagen molecules at different positions in the channels with high resolution. We collect the X-ray diffraction patterns for different flow rates, flow rate ratios, and $\mathrm{NaOH}$ concentrations while keeping the input collagen concentration constant at $10 \mathrm{mg} / \mathrm{mL}$ in $0.075 \mathrm{M} \mathrm{AcH}$. For the X-ray experiments the channel width is $d=150 \mu \mathrm{m}$ and the depth $h=300 \mu \mathrm{m}$.

During X-ray measurements we cannot directly visualize the conditions in the microchannels. Thus, we conduct appropriate FEM simulations for the parameters in order to relate the measured X-ray signal to the hydrodynamic, diffusive, and $\mathrm{pH}$ 
related phenomena in the channel. In figure 6.117 the simulated viscosity (upper half of the image) and $\mathrm{pH}$ distribution (lower half) is shown. The flow rate ratio is $v_{\text {main }}: v_{\text {side }}=1: 1.5$ and the absolute flow rates are $v_{\text {main }}=840 \mu \mathrm{m} / \mathrm{s}$ and $v_{\text {side }}=1260 \mu \mathrm{m} / \mathrm{s}$. The $\mathrm{NaOH}$ concentration is $c_{\mathrm{NaOH}}=1 \mathrm{M}$. Compared to the parameters used for the birefringence experiments (see for example figure 6.5b) a much lager fraction of the center stream contains assembled collagen and is therefore highly viscous. We collect X-ray diffraction images in different positions in the center of the main channel, as noted in figure $6.11 \mathrm{a}$ by circles. The corresponding intensities are plotted against the $q$-vector in figure 6.11b. Intensity plots are obtained by an azimuthal integration of the two-dimensional diffraction images, two examples of which are shown in figure 6.11k. Directly in the center of the crossed channels a peak at $q=0.146 \AA^{-1}$ is observed, which corresponds to a spacing of $d=2 \pi / q=4.3 \mathrm{~nm}$. Thus, a supramolecular structure can be found in the system yielding a characteristic length scale of $d=4.3 \mathrm{~nm}$. Further down in the main channel the peak vanishes.

We also measured the X-ray signal at different positions perpendicular to the main channel (figure 6.12). In this case the flow rate ratio is $v_{\text {main }}: v_{\text {side }}=1: 2.4$ and the absolute flow rates are $v_{\text {main }}=1050 \mu \mathrm{m} / \mathrm{s}$ and $v_{\text {side }}=2520 \mu \mathrm{m} / \mathrm{s}$. The NaOH concentration is $c_{\mathrm{NaOH}}=0.5 \mathrm{M}$. Again, a distinct correlation peak is observed in the center of the cross and is even more pronounced at $y=30 \mu \mathrm{m}$. In the side channel, at $y=120 \mu \mathrm{m}$, there is no collagen present and consequently no observable peak.

a)
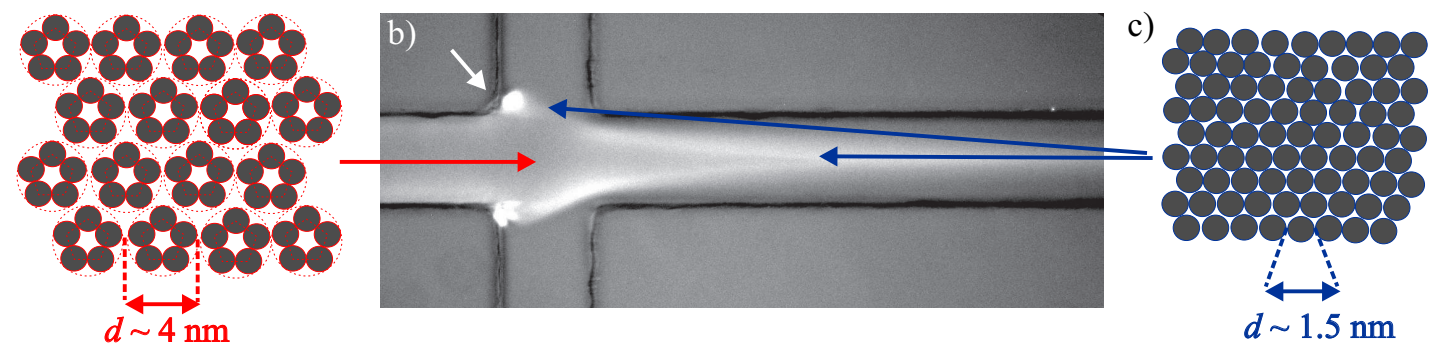

Figure 6.13: Schematic representation of the model for the different liquid crystalline phases of collagen forming during fibrillogenesis. a) The peak at $d=4.3 \mathrm{~nm}$ may indicate the formation of critical subunits which in turn are packed in a hexagonal lattice. b) Birefringence micrograph indicating the regions of different molecular packing. c) In the regions of strongest birefringence signal, collagen molecules are possibly laterally packed in a quasi-hexagonal lattice with a spacing of about $d=1.5 \mathrm{~nm}$.

For comparison and additional visualization of the conditions which are used in the $\mathrm{X}$-ray experiments, a birefringence micrograph for a flow rate ratio $v_{\text {main }}: v_{\text {side }}=1: 1$ and a $c_{\mathrm{NaOH}}=1 \mathrm{M}$ is shown in figure $6.13 \mathrm{~b}$. The large agglomerates of collagen in the corners of the crossed channels (white arrow) are due to the extremely increased 
self-assembly rate in these regions and the channel walls that serve as nucleation sites. The aggregates, however, do not influence the flow profile, as can also be seen in figure $6.13 \mathrm{~b}$.

Interestingly, we do not observe the most intense X-ray peak in the regions where we detect the brightest birefringence signal in corresponding polarized light microscopy experiments, that is to say, in those positions where the viscosity of the collagen solution is strongly increased due to self-assembly at $\sim \mathrm{pH} 6$. In contrast, the strongest $\mathrm{X}$-ray peak is detected at the center region (red arrow in figure $6.13 \mathrm{~b}$ ), where we observe a correlation peak at $d=4.3 \mathrm{~nm}$. X-ray measurements on in vivo systems such as rat tail tendon [58] or cornea [60] have revealed a diameter for the subunits that form from the collagen molecules of around $4 \mathrm{~nm}$, which is in agreement with our result. Moreover, the characteristic spacing is consistent with the model for the packing of type I collagen molecules in the native fibril, which has been suggested by Piez and Trus in 1981. [57] They proposed that in a first step of collagen selfassembly, five-stranded microfibrils form and eventually grow linearly in a staggered manner (see also section 2.2.1). It is well possible that in the center region of the cross we have $\mathrm{pH}$ conditions $(\sim 4-5)$ that lead to the formation of pentameric subunits, which in turn are arranged in a hexagonal lattice.

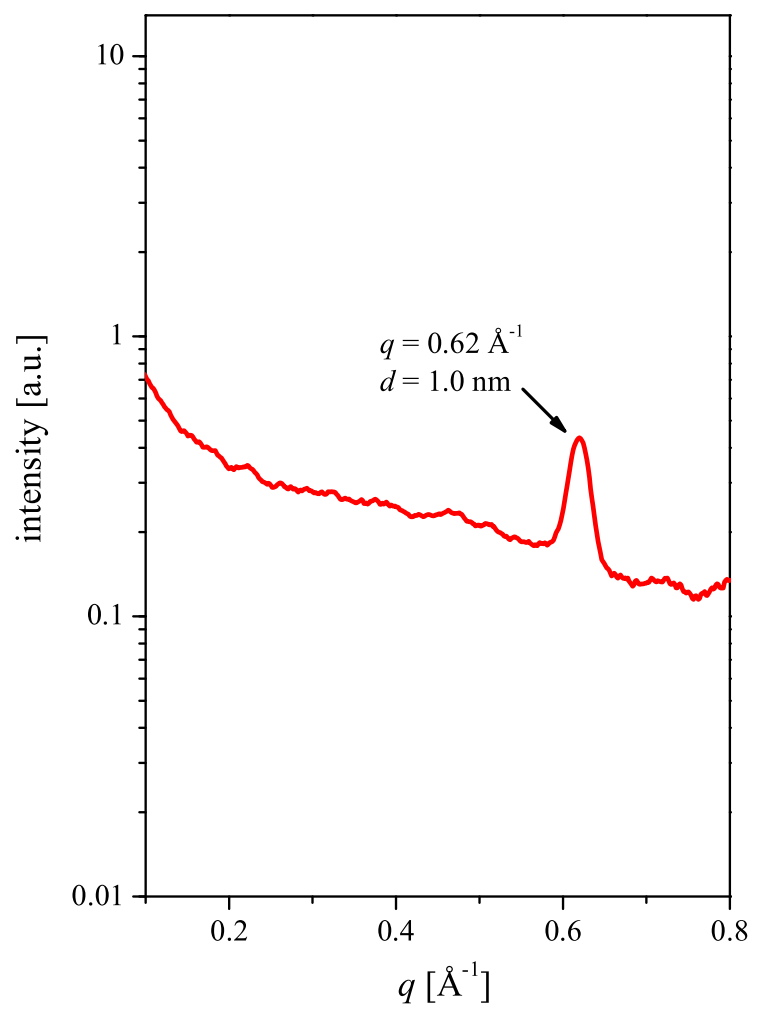

Figure 6.14: X-ray scan of bulk collagen sample. The peak at $d=1.0 \mathrm{~nm}$ may indicate that the collagen molecules are close packed in a hexagonal lattice. 
We detect either no or merely a weak X-ray signal when measuring at the positions within the crossed channels where we would expect the strongest birefringence based on the microscopy experiments (blue arrows in figure 6.13b). From comparison of polarized light microscopy experiments and simulations we know that a strong birefringence signal is correlated with highly viscous collagen solution which, in turn, indicates that the molecules are assembled in larger aggregates. Bulk experiments $[125,126]$ and theoretical studies $[54,57]$ have shown that upon further lateral compression previously formed pentameric subunits disappear and a near-hexagonal lattice of the molecules forms. This arangement is sketched in figure 6.13c. A possible explanation of the experimental results is that in the highly birefringent regions this quasi-hexagonal phase has already formed. The $q$-range of our setup at the synchrotron is $0.02-0.35 \AA^{-1}$, corresponding to $d=1.8-31.4 \mathrm{~nm}$. Since the spacing of the close packed hexagonal phase is similar to the diameter of the collagen molecules $(\sim 1.5 \mathrm{~nm})$, we do not observe a corresponding signal in the experiments. The in-house Bruker AXS Nanostar proves to be more flexible concerning the $q$ range. We prepare bulk samples containing collagen $\left(c_{\text {collagen }}=5 \mathrm{mg} / \mathrm{mL}\right), \mathrm{AcH}$ $\left(c_{A c H}=0.0375 \mathrm{M}\right)$, and $\mathrm{NaOH}\left(c_{N a O H}=0.05 \mathrm{M}\right)$ with $\sim \mathrm{pH} 10$. The solution is filled into quartz capillaries and placed in the evacuated measuring chamber. The vacuum leads to evaporation of the water in the system and thus to an increase in collagen, $\mathrm{AcH}$, and $\mathrm{NaOH}$ concentration. In figure 6.14 an X-ray scan for an exposure time of $10,000 \mathrm{~s}$ of bulk collagen is shown. We observe a peak at $d=1.0 \mathrm{~nm}$ (see figure 6.14). We are not able to exactly determine the collagen concentration at the measuring time point but estimate it to $100-500 \mathrm{mg} / \mathrm{mL}$. These results provide evidence that indeed at $\mathrm{pH} \gtrsim 6$, which leads to a highly viscous, strongly birefringent collagen gel, we deal with a close packed hexagonal phase with a spacing on the order of the single collagen molecule diameter.

Although such bulk experiments can be used to investigate the system, we are able to realize only one $\mathrm{pH}$ condition at a time. Thus, systematic analysis of the complete process proves to be very complex and time consuming. In contrast, the experiments that we conduct in microchannels allow for the establishment of $\mathrm{pH}$ gradients which in turn enable us to perform non-equilibrium measurements. By measuring the system at different positions in the channels we obtain information about different stages of fibril formation. The well defined controllability of the conditions via adjustment of concentrations and flow rates provides an excellent tool for the systematic study of reacting biological systems. 


\subsection{Future Directions}

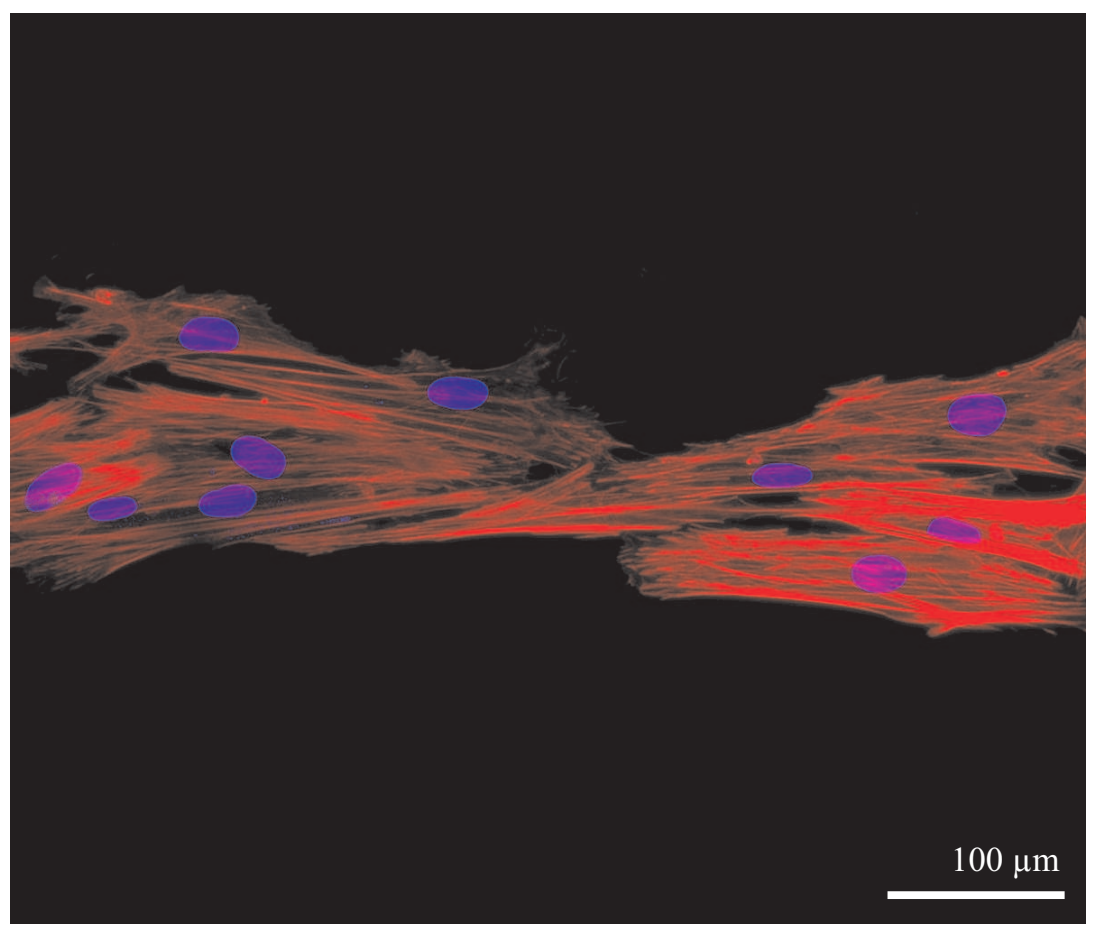

Figure 6.15: Fluorescence micrograph of VSMC grown on a substrate with aligned collagen. The actin cytoskeleton shows a preferred orientation in the direction of the alignment (horizontal in the image)

The importance of investigations of collagen fibril formation and the influence of alignment is not only relevant from a fundamental point of view. The internal structure of collagen aggregates is also known to influence cell arrangement in physiological systems. In blood vessels, for example, the smooth muscle cells (VSMC) are circumferentially oriented as are the collagen fibrils constituting the ECM that surrounds them. It is not clear, though, whether the cells orient the collagen or vice versa. One possibility is that cells grow on an aligned collagen matrix just as on "tracks" which would lead to oriented aligned cells. Another possibility is that the originally unoriented collagen is reoriented by the cells and the aligned cells subsequently produce aligned collagen. Most likely there is interaction and interplay of both scenarios. [4] In order to better understand the involved mechanisms we have started to use microfluidic methods to prepare aligned collagen substrates and grow cells on them. In figure 6.15 a fluorescence micrograph of such an experiment is shown. The actin cytoskeleton of the VSMC is stained with a red fluorescent dye and the cell nucleus is stained with a blue dye. In the image, the direction of collagen alignment is horizontal. Obviously, the actin cytoskeleton is preferably oriented in 
parallel to this direction. This is an indication that the cells indeed use the collagen fibrils as tracks to adjust the preferred orientation of their actin stress fibers. In order to mimic systems found in nature the principle mechanisms and concepts have to be investigated in a controlled environment. Eventually, such experiments may provide deeper insight into cell-ECM interactions and advance the field of tissue engineering. 


\section{Chapter 7}

\section{Conclusions}

Two different biopolymers are studied both of which belong to the most abundant building blocks in mammals: the cytoskeletal protein actin and the extracellular matrix protein collagen. A deeper fundamental insight into the structural and functional properties of these fibrous proteins is essential for a further understanding of cellular and tissue mechanics. As a principle tool for the manipulation and analysis of the biomacromolecules we use microfluidic techniques to take advantage of the small-scale controllability and the possibility to mimic physical as well as chemical conditions of physiological systems in a discriminating manner. This technique proves to be well suited for the investigation of single molecules as well as in situ study of self-assembly in semidilute polymer solutions.

In the first part of this thesis, single molecule experiments on actin filaments under the influence of confining microchannels are presented. We study the biopolymers by means of fluorescence microscopy and systematically vary different parameters, yet ensuring that the system can be analytically described. We measure the persistence length $L_{P}$ of unconfined as well as confined actin filaments fluctuating in two dimensions by considering the bending energy of the biopolymers. The influence of geometric confinement on the mechanics of F-actin is analyzed in terms of tangent correlation functions, radial distribution functions, and segment distributions. We find that the behavior of single biopolymers depends strongly on their contour length as well as on the degree of confinement which is determined by the dimensions and geometry of the microfluidic environment. The influence of the restraining channel walls on the actin filaments can be well described by the competition of bending energy and confining energy which we approximate as a parabolic potential. We perform data analysis in terms of different scaling laws known from polymer physics. Thus, the behavior of the filaments near the channel walls can characterized by the 
deflection length $\lambda$. The segment distribution of the macromolecules in the channels is also successfully compared to the corresponding scaling laws. Altogether, the experimental results, the analytical description, and a comparison to Monte Carlo simulations yield a consistent characterization of the biosystem. Thus, we are able to mimic the natural environment of F-actin which, in the cell, is embedded in a tight network of fibrous cytoskeletal proteins. Apart from its considerable importance in life science, actin is one of the few experimentally accessible model systems for semiflexible polymers. The results presented here can therefore be applied to polymer physics in general and yield interesting results especially for the intermediate region of stiffness. Moreover, the experiments emphasize that microfluidics provides an excellent method to study single molecule systems while testing a large pool of individuals in order to obtain accurate statistics.

In the second part, we present in situ studies on the self-assembly of collagen in a stable and well-defined $\mathrm{pH}$ gradient. We use a microfluidic mixing and hydrodynamically focusing device to establish controllable $\mathrm{pH}$ conditions that allow for non-equilibrium measurements in the microchannels. Owing to small length scales and high viscosity, the system is governed by laminar flow. This unique property allows for the observation of different stages of the reaction at different positions in the channel. Semidilute collagen solution that is brought into the gradient starts to self-assemble once a neutral $\mathrm{pH}$ is reached. Polarized light microscopy is used to visualize this evolution of liquid crystalline phases in the continuous flow. Assembled collagen has a very high viscosity which in turn influences the hydrodynamic phenomena in the microfluidic device. In order to quantify the results we conduct finite element method simulations of the system. For this purpose, we solve the stationary incompressible Navier-Stokes equation and the appropriate diffusion equations. By coupling the viscosity to the local $\mathrm{pH}$ we are able to locate regions where the collagen is self-assembled. In turn, this method allows for measurement of the viscosity of minute quantities of biological material. In order to study the system on a molecular and nanometer scale, X-ray microdiffraction experiments are conducted. The continuous flow in our setup enables us to perform such experiments without material damage. We are able to dynamically observe the evolution of the formation of critical subunits for collagen fibrillogenesis and relate their incidence to the $\mathrm{pH}$ conditions in the channels. Thus we obtain interesting information about the key-factors for hierarchical self-assembly of collagen. From a more applied point of view, the development of engineered tissues is among the most important missions of biomedicine. Fundamental research on structural and functional properties of collagenic tissue are crucial for deeper insights and a better understanding of the 
biological systems which are to be mimicked.

The systems studied here are inherently important for biology and biomedicine. However, the results derived from the investigation of single filamentous proteins, on the one hand, and self-assembly processes, on the other hand, are remarkable also from a more fundamental, biophysical point of view. The underlying concepts may be generalized to a large pool of systems. Furthermore, the microfluidic methods that are established are applicable to a variety of questions concerning life science. 


\section{Appendix A}

\section{List of Abbreviations}

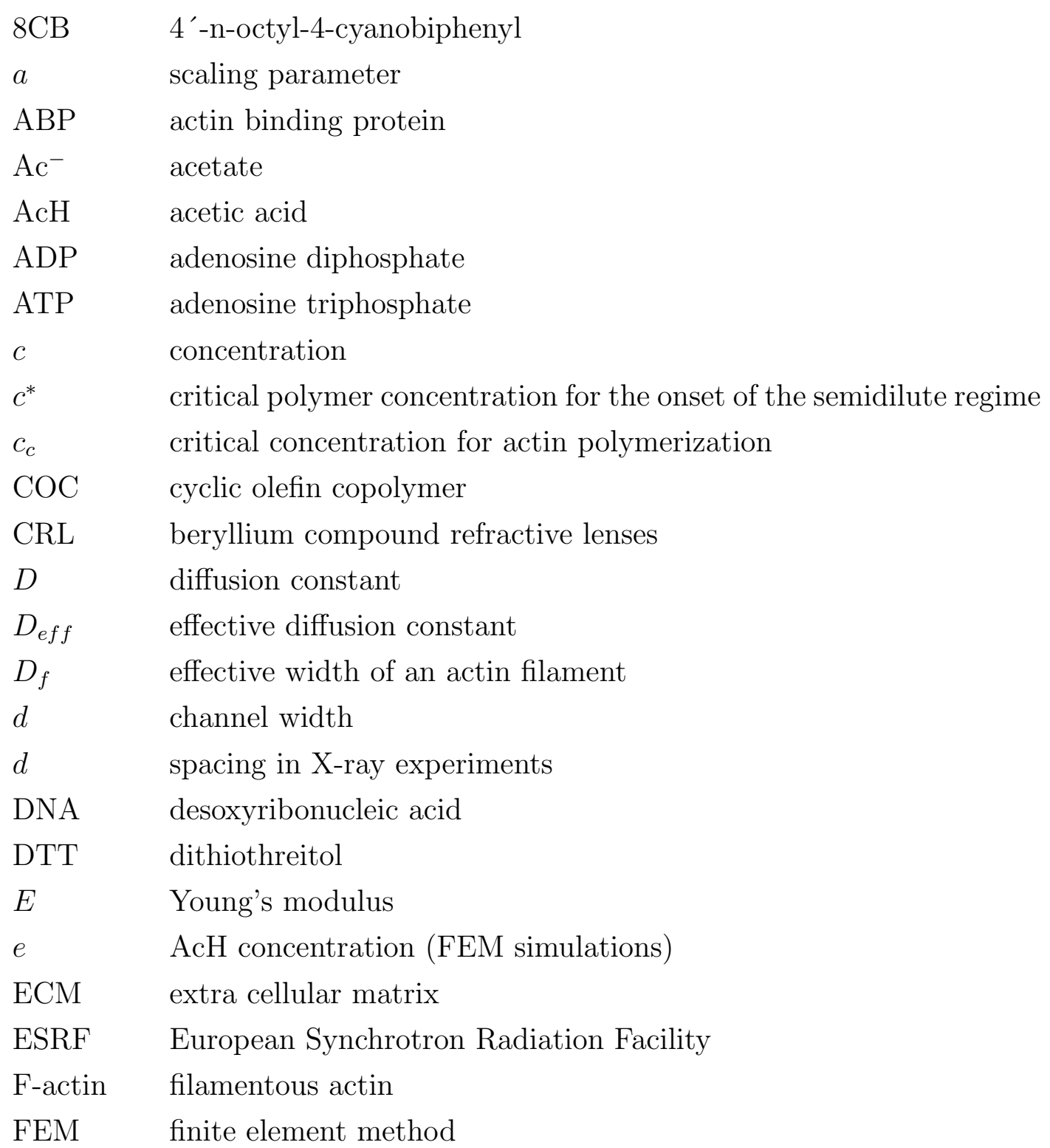


FWHM full width at half maximum

G-actin globular, monomeric actin

$h \quad$ channel depth

I geometrical moment of inertia

$K \quad$ potential strength

$K_{s} \quad$ acidity constant

$K_{w} \quad$ dissociation constant of water

$k_{B} \quad$ Boltzmann constant

$k_{o n} \quad$ rate constant for the addition of actin monomers to a filament

$k_{\text {off }} \quad$ rate constant for the subtraction of actin monomers from a filament

$L \quad$ contour length

$L_{P} \quad$ persistence length

$L_{x} \quad$ projected contour length

$l \quad$ arc length

$l_{t} \quad$ length of tangent vectors

$M_{W} \quad$ molecular weight

$N \quad$ number of individual measurements

$n \quad \mathrm{NaOH}$ concentration (FEM simulations), divided by $e$

$N_{A} \quad$ Avogadro's number

N.A. numerical aperture

$\mathrm{NaOH}$ sodium hydroxyide

$\mathrm{OH}^{-} \quad$ hydroxide

$p \quad$ pressure

PDMS poly(dimethysiloxane)

$R \quad$ end-to-end distance

R end-to-end vector

$R_{c} \quad$ radius of curvature

$R_{g} \quad$ radius of gyration

$R_{i} \quad$ inner radius of curvature

$R_{o} \quad$ outer radius of curvature

$r_{1}, r_{2} \quad$ fit parameters

$\mathbf{r} \quad$ position vector

Re Reynolds number

SDS-page sodium-dodecyl-sulfate-polyacrylamide gel electrophoresis

$T$ temperature

t tangent vector

Tris-HCl tris(hydroxymethyl)aminomethane-hydrochloride 


$\begin{array}{ll}\text { TRITC } & \text { tetramethylrhodamine-isothiocyanate } \\ v_{c m} & \text { center of mass velocity } \\ v_{\text {main }} & \text { flow velocity in the main inlet channel } \\ v_{\text {side }} & \text { flow velocity in the side inlet channel } \\ \mathbf{v} & \text { velocity } \\ \text { VSMC } & \text { vascular smooth muscle cells } \\ W & \text { energy } \\ \text { WLC } & \text { worm-like chain } \\ \Delta & \text { period of the stagger in collagen fibrils } \\ \zeta & \text { thermal roughness exponent } \\ \eta & \text { viscosity } \\ \theta_{r} & \text { segment distribution exponent } \\ \kappa & \text { bending rigidity } \\ \Lambda & \text { wavelength } \\ \lambda & \text { deflection length } \\ \lambda & \text { wavelength } \\ \lambda^{*} & \text { deflection length } \\ \lambda \text {-DNA } & \text { lambda bacteriophage DNA } \\ \mu \text { TAS } & \text { total analysis systems } \\ \rho & \text { density } \\ \tau & \text { total observation time } \\ \phi & \text { volume fraction of polymer in solution } \\ \chi & \text { form factor } \\ \chi & \text { segment distribution exponent } \\ & \end{array}$




\section{Bibliography}

[1] E. Schrödinger. What is life? Cambridge University Press, 1944.

[2] S. Paulsen and A. Tsiaras. Unser Herz - neue Einsichten in das Organ des Lebens. GEO, 4:148-174, 2006.

[3] H. Lodish, A. Berk, P. Matsudaira, C.A. Kaiser, M. Krieger, M.P. Scott, S.L. Zipursky, and J. Darnell. Molecular Cell Biology. W.H. Freeman and Company, New York, fifth edition, 2004.

[4] B. Alberts, A. Johnson, J. Lewis, M. Raff, K. Roberts, and P. Walter. Molecular Biology of the Cell. Garland Science, New York, fourth edition, 2002.

[5] T. Thorsen, S.J. Maerkl, and S.R. Quake. Microfluidic Large-Scale Integration. Science, 298:580-584, 2002.

[6] R. Bunk, J. Klinth, L. Montelius, I.A. Nicholls, P. Omling, S. Tågerud, and A. Månsson. Actomyosin motility on nanostructured surfaces. Biochemical and Biophysical Research Communications, 301(3):783-788, 2003.

[7] W. Reisner, K.J. Morton, R. Riehn, Y.M. Wang, Z. Yu, M. Rosen, J.C. Sturm, S. Chou, E. Frey, and R.H. Austin. Statics and Dynamics of Single DNA Molecules Confined in Nanochannels. Physical Review Letters, 94(19):196101, 2005.

[8] J.O. Tegenfeldt, C. Prinz, H. Cao, S. Chou, W.W. Reisner, R. Riehn, Y.M. Wang, E.C. Cox, J.C. Sturm, P. Silberzan, and R.H. Austin. The dynamics of genomic-length DNA molecules in 100-nm channels. Proceedings of the National Academy of Sciences of the United States of America, 101(30):1097910983, 2004.

[9] J. Clemmens, H. Hess, J. Howard, and V. Vogel. Analysis of Microtubule Guidance in Open Microfabricated Channels Coated with the Motor Protein Kinesin. Langmuir, 19(5):1738-1744, 2003. 
[10] D.E. Smith and S. Chu. Response of Flexible Polymers to a Sudden Elongational Flow. Science, 281:1335-1340, 1998.

[11] P.K. Wong, Y.-K. Lee, and C.-M. Ho. Deformation of DNA molecules by hydrodynamic focusing. Journal of Fluid Mechanics, 497:55-65, 2003.

[12] P.J. Shrewsbury, S.J. Muller, and D. Liepmann. Effect of Flow on Complex Biological Macromolecules in Microfluidic Devices. Biomedical Microdevices, 3(3):225-238, 2001.

[13] P.-G. de Gennes. Scaling Concepts in Polymer Physics. Cornell University Press, Ithaca, 1979.

[14] T. Odijk. On the Statistics and Dynamics of Confined or Entangled Stiff Polymers. Macromolecules, 16:1340-1344, 1983.

[15] M. He, J.S. Edgar, G.D.M. Jeffries, R.M. Lorenz, J.P. Shelby, and D.T. Chiu. Selective Encaspsulation of Single Cells and Subcellular Organelles into Picoliter- and Femtoliter-Volume Droplets. Analytical Chemistry, 77:1539$1544,2005$.

[16] T. Pfohl and S. Herminghaus. Mikrofluidik mit komplexen Flüssigkeiten. Physik Journal, 2(1):35-40, 2003.

[17] T. Pfohl, F. Mugele, R. Seemann, and S. Herminghaus. Trends in Microfluidics with Complex Fluids. ChemPhysChem, 4(12):1291-1298, 2003.

[18] I.Y. Wong, M.L. Gardel, D.R Reichman, E.R Weeks, M.T. Valentine, A.R. Bausch, and D.A. Weitz. Anomalous Diffusion Probes Microstructure Dynamics of Entangled F-Actin Networks. Physical Review Letters, 92(17):178101, 2004.

[19] D. Boal. Mechanics of the Cell. Cambridge University Press, 2001.

[20] J. Howard. Mechanics of Motor Proteins and the Cytoskeleton. Sinauer, Sunderland, 2001.

[21] L. Le Goff, O. Hallatscheck, E. Frey, and F. Amblard. Tracer Studies on F-Actin Fluctuations. Physical Review Letters, 89(25):258101, 2002.

[22] A. Ott, M. Magnasco, A. Simon, and A. Libchaber. Measurement of the persistence length of polymerized actin using fluorescence microscopy. Physical Review E, 48(3):R1642-R1645, 1993. 
[23] T. Yanagida, M. Nakase, K. Nishiyama, and F. Oosawa. Direct observation of motion of single F-actin filaments in the presence of myosin. Nature, 307:5860, 1984 .

[24] J. Käs, H. Strey, J. X. Tang, D. Finger, R. Ezzell, E. Sackmann, and P. A. Janmey. F-Actin, a Model Polymer for Semiflexible Chains in Dilute, Semidilute and Liquid Crystalline Solutions. Biophysical Journal, 70:609-625, 1996.

[25] F. Gittes, B. Mickey, J. Nettleton, and J. Howard. Flexural Rigidity of Microtubules and Actin Filaments Measured from Thermal Fluctuations in Shape. The Journal of Cell Biology, 120(4):923-934, 1993.

[26] H. Isambert, P. Venier, A.C. Maggs, A. Fattoum, R. Kassab, D. Pantaloni, and M.-F. Carlier. Flexibility of Actin Filaments Derived from Thermal Fluctuations. Effect of Bound Nucleotide, Phalloidin, and Muscle Regulatory Proteins. The Journal of Biological Chemistry, 270(19):11437-11444, 1995.

[27] D. Riveline, C.H. Wiggins, R.E. Goldstein, and A. Ott. Elastohydrodynamic study of actin filaments using fluorescence microscopy. Physical Review E, 56(2):R1330-R1333, 1997.

[28] G.C.L. Wong, J.X. Tang, A. Lin, Y. Li, P.A. Janmey, and C.R. Safinya. Hierarchical Self-Assembly of F-Actin and Cationic Lipid Complexes: Stacked Three-Layer Tubule Networks. Science, 288:2035-2039, 2000.

[29] N.F. Bouxsein, L.S. Hirst, Y. Li, C.R. Safinya amd Z.A. Samah, N.C. MacDonald, and R. Pynn. Alignment of filamentous proteins and associated molecules through confinement in microchannels. Applied Physics Letters, 85(23):5775$5777,2004$.

[30] O. Pelletier, E. Pokidysheva, L.S. Hirst, N. Bouxsein, Y. Li, and C.R. Safinya. Structure of Actin Cross-Linked with $\alpha$-Actinin: A Network of Bundles. Physical Review Letters, 91(14):148102, 2003.

[31] J.X. Tang and P.A. Janmey. The Polyelectrolyte Nature of F-actin and the Mechanism of Actin Bundle Formation. The Journal of Biological Chemistry, 271(15):8556-8563, 1995.

[32] P.A. Janmey, S. Hvidt, J. Käs, D. Lerche, A. Maggs, E. Sackmann, M. Schliwa, and T.P. Stossel. The Mechanical Properties of Actin Gels. Elastic Modulus and Filament Motions. The Journal of Biological Chemistry, 51:32503-32513, 1994. 
[33] J. Xu, W.H. Schwarz, J.A. Käs, T.P. Stossel, P.A. Janmey, and T.D. Pollard. Mechanical Properties of Actin Filament Networks Depend on Prepapration, Polymerization Conditions, and Storage of Actin Monomers. Biophysical Journal, 74:2731-2740, 1998.

[34] A. Kakugo, S. Sugimoto, J.P. Gong, and Y. Osada. Gel Machines Constructed from Chemically Cross-linked Actins and Myosins. Advanced Materials, 14(16):1124-1126, 2002.

[35] J. Wilhelm and E. Frey. Radial Distribution Function of Semiflexible Polymers. Physical Review Letters, 77(12):2581-2584, 1996.

[36] P. Levi. Actin Filaments in Confined Geometry. Diploma Thesis, University of Erlangen, 2005.

[37] F. Brochard-Wyart, T. Tanaka, N. Borghi, and P.-G. de Gennes. Semiflexible Polymers Confined in Soft Tubes. Langmuir, 21:4144-4148, 2005.

[38] L. Harnau and P. Reineker. Equilibrium and dynamical properties of semiflexible chain molecules with confined transverse fluctuations. Physical Review E, 60(4):4671-4676, 1999.

[39] T.W. Burkhardt. Free energy of a semiflexible polymer in a tube and statistics of a randomly-accelerated particle. Journal of Physics A: Mathematical and General, 30:L167-L172, 1997.

[40] T. Odijk. Similarity Applied to the Statistics of Confined Stiff Polymers. Macromolecules, 17:502-503, 1984.

[41] M. Dijkstra, D. Frenkel, and H.N.W. Lekkerkerker. Confinement free energy of semiflexible polymers. Physica A, 193:374-393, 1993.

[42] T.W. Burkhardt. Free energy of a semiflexible polymer confined along an axis. Journal of Physics A: Mathematical and General, 28:L629-L635, 1995.

[43] B. Hinner, M. Tempel, E. Sackmann, K. Kroy, and E. Frey. Entanglement, Elasticity, and Viscous Relaxation of Actin Solutions. Physical Review Letters, 81(12):2614-2617, 1998.

[44] J. Kierfeld, T. Kühne, and R. Lipowsky. Discontinuous Unbinding Transitions of Filament Bundles. Physical Review Letters, 95:038102, 2005. 
[45] S. Köster, J. Kierfeld, H. Stark, S. Herminghaus, and T. Pfohl. Thermal Fluctuations of Individual Semiflexible Polymers in Confined Geometry. condmat, 0509548, 2005.

[46] S. Köster, D. Steinhauser, and T. Pfohl. Brownian motion of actin filaments in confining microchannels. Journal of Physics: Condensed Matter, 17:S4091S4104, 2005.

[47] C. Ploetz, E.I. Zycband, and D.E. Birk. Collagen fibril assembly and deposition in the developing dermis: Segmental deposition in extracellular compartments. Journal of Structural Biology, 106(1):73-81, 1991.

[48] http://www.sju.edu/biology/ksweb/microsc/steve03.jpg.

[49] R.G. Thakar, F. Ho, N.F. Huang, D. Liepmann, and S. Li. Regulation of vascular smooth muscle cells by micropatterning. Biochemical and Biophysical Research Communications, 307:883-890, 2003.

[50] J. Engel and D.J. Prockop. The Zipper-Like Folding of Collagen Triple Helices and the Effects of Mutations that Disrupt the Zipper. Annual Review of Biophysics and Biophysical Chemistry, 20:137-152, 1991.

[51] R.A. Berg, D.E. Birk, and F.H. Silver. Physical characterization of type I procollagen in solution: evidence that the propeptides limit self-assembly. International Journal of Biological Macromolecules, 8(3):177-182, 1986.

[52] F.H. Silver, J.W. Freeman, and G.P. Seehra. Collagen self-assembly and the development of tendon mechanical properties. Journal of Biomechanics, 36:1529-1553, 2003.

[53] A.J. Hodge and J.A. Petruska. Recent studies with the electron microscope on ordered aggregates of the tropocollagen molecule. In Aspects of Protein Chemistry, pages 289-300. Academic Press, London, 1963.

[54] T.J. Wess, A.P. Hammersley, L. Wess, and A. Miller. A Consensus Model for Molecular Packing of Type I Collagen. Journal of Structural Biology, 122:92100, 1998.

[55] J.P.R.O. Orgel, A. Miller, T.C. Irving, R.F. Fischetti, A.P. Hammersley, and T.J. Wess. The In Situ Supermolecular Structure of Type I Collagen. Structure, 9:1061-1069, 2001. 
[56] J.W. Smith. Molecular Pattern in Native Collagen. Nature, 219:157-158, 1968.

[57] K.A. Piez and B.L. Trus. A new model for packing of type-I collagen molecules in the native fibril. Bioscience reports, 1:801-810, 1981.

[58] A. Miller and J.S Wray. Molecular Packing in Collagen. Nature, 230:437-439, 1971.

[59] A. Miller and D.A.D. Parry. Structure and packing of microfibrils in collagen. Journal of Molecular Biology, 75(2):441-447, 1973.

[60] D.F. Holmes, C.J. Gilpin, C. Baldock, U. Ziese, A.J. Koster, and K.E. Kadler. Corneal collagen firbril structure in three dimensions: Structural insights into fibril assembly, mechanical properties, and tissue organization. Proceedings of the National Academy of Sciences of the United States of America, 98(13):7307-7312, 2001.

[61] D.L. Christiansen, E.K. Huang, and F.H. Silver. Assembly of type I collagen: fusion of fibril subunits and the influence of fibril diameter on mechanical properties. Matrix Biology, 19:409-420, 2000.

[62] Y.C. Fung. Biomechanics: Mechanical Properties of Living Tissues. Springer, New York, 1993.

[63] H. Haga, C. Irahara, R. Kobayashi, T. Nakagaki, and K. Kawabata. Collective Movement of Epithelial Cells on a Collagen Gel Substrate. Biophysical Journal, 88:2250-2256, 2005.

[64] N.S. Murthy. Liquid crystallinity in collagen solutions and magnetic orientation of collagen fibrils. Biopolymers, 23(7):1261-1267, 1984.

[65] S. Guido and R.T. Tranquillo. A methodology for the systematic and quantitative study of cell contact guidance in oriented collagen gels. Journal of Cell Science, 105:317-331, 1993.

[66] F. Jiang, H. Hörber, J. Howard, and D.J. Müller. Assembly of collagen into microribbons: effects of $\mathrm{pH}$ and electrolytes. Journal of Structural Biology, 148:268-278, 2004.

[67] D.L. Wilson, R. Martin, S. Hong, M. Cronin-Golomb, C.A. Mirkin, and D.L. Kaplan. Surface organization and nanopatterning of collagen by dip-pen nanolithography. Proceedings of the National Acadamy of Sciences of the United States of America, 98(24):13660-13664, 2001. 
[68] D.P. Knight and D. Feng. Interaction of collagen with hydrophobic protein granulates in the egg capsule of the dogfish scyliorhinus canicula. Tissue and Cell, 26(2):155-167, 1994.

[69] D.O. Freytes, S.F. Badylak, T.J. Webster, L.A. Geddes, and A.E. Rundell. Biaxial strength of multilaminated extracellular matrix scaffolds. Biomaterials, 25:2353-2361, 2004.

[70] C. Stamm, A. Khosravi, N. Grabow, K. Schmohl, N. Treckmann, A. Drechsel, M. Nan, K.-P. Schmitz, A. Haubold, and G. Steinhoff. Biomatrix/Polymer Composite Material for Heart Valve Tissue Engineering. Annals of Thoracic Surgery, 78:2084-2093, 2004.

[71] E.D. Boland, J.A. Matthews, K.J. Pawlowski, D.G. Simpson, G.E. Wnek, and G.L. Bowlin. Electrospinning collagen and elastin: preliminary vascular tissue engineering. Frontier in Bioscience, 9:1422-1432, 2004.

[72] W. Tan and T.A. Desai. Layer-by-layer microfluidics for biomimetic threedimensional structures. Biomaterials, 255:1355-1364, 2004.

[73] S.M. O'Connor, D.M. Stenger, K.M. Shaffer, and W. Ma. Survival and neurite outgrowth of rat cortical neurons in three-dimensional agarose and collagen gel matrices. Neuroscience Letters, 304:189-193, 2001.

[74] C. Gaudet, W.A. Marganski, S. Kim, C.T. Brown, V. Gunderia, M. Dembo, and J.Y. Wong. Influence of Type I Collagen Surface Density on Fibroblast Spreading, Motility, and Contractility. Biophysical Journal, 85:3329-3335, 2003.

[75] J.M. McPehrson, D.G. Wallace, S.J. Sawamura, A. Conti, R.A. Condell, S. Wade, and K.A. Piez. Collagen Fibrillogenesis In Vitro: A Characterization of Fibril Quality as a Function of Assembly Conditions. Collagen Related Research, 5:119-135, 1985.

[76] S.L. Voytik-Harbin, B.A. Roeder, J.E. Sturgis, K. Kokini, and J.P. Robinson. Simultaneous Mechanical Loading and Confocal Reflection Microscopy for Three-Dimensional Microbiomechanical Analysis of Biomaterials and Tissue Constructs. Microscopy Microanalysis Microstructures, 9:74-85, 2003.

[77] B.A. Roeder, K. Kokini, J.E. Sturgis, J.P. Robinson, and S.L. Voytik-Harbin. Tensile Mechanical Properties of Three-Dimensional Type I Collagen Extra- 
cellular Matrices with Varied Microstructure. Journal of Biomechanical Engineering, 124:214-222, 2002.

[78] A. Wegner. Treadmilling of actin at physiological salt concentrations. An analysis of the critical concentrations of actin filaments. Journal of Molecular Biology, 161(4):607-615, 1982.

[79] S.R. Quake and A. Scherer. From Micro- to Nanofabrication with Soft Materials. Science, 290:1536-1540, 2000.

[80] D.C. Duffy, J.C. McDonald, O.J.A. Schueller, and G.M. Whitesides. Rapid Prototyping of Microfluidic Systems in Poly(dimethylsiloxane). Analytical Chemistry, 70(23):4974-4984, 1998.

[81] E. Delamarche, A. Bernard, H. Schmid, A. Bietsch, B. Michel, and H. Biebuyck. Microfluidic Networks for Chemical Patterning of Substrates: Design and Application to Bioessays. Journal of the American Chemical Society, 120(3):500-508, 1998.

[82] Y. Xia and G.M. Whitesides. Soft Lithography. Angewandte Chemie International Edition, 37:550-575, 1998.

[83] J.M. Watson and M.G. Baron. The behaviour of water in poly(dimethylsiloxane). Journal of Membrane Science, 110:47-57, 1996.

[84] E. Verneuil, A. Buguin, and P. Silberzan. Permeation-induced flows: Consequences for silicone-based microfluidics. Europhysics Letters, 68(3):412-418, 2004.

[85] J.B. Knight, A. Vishwanath, J.P. Brody, and R.H. Austin. Hydrodynamic Focusing on a Silicon Chip: Mixing Nanoliters in Microseconds. Physical Review Letters, 80(17):3863-3866, 1998.

[86] A. Otten, S. Köster, B. Struth, A. Snigirev, and T. Pfohl. Microfluidics of soft matter investigated by small-angle X-ray scattering. Journal of Synchrotron Radiation, 12:745-750, 2005.

[87] C.R. Cabrera, B. Finlayson, and P. Yager. Formation of Natural pH Gradients in a Microfluidic Device under Flow Conditions: Model and Experimental Validation. Analytical Chemistry, 73:658-666, 2001. 
[88] M.S. Munson and P. Yager. Simple quantitative optical method for monitoring the extent of mixing applied to a novel microfluidic mixer. Analytica Chimica Acta, 507:63-71, 2004.

[89] Olympus ${ }^{\circledR}$. UIS Fluorescence Mirror Units Technical Specifications.

[90] http://www.ahf.de.

[91] http://probes.invitrogen.com/servlets/spectra/.

[92] A. Otten. private communications.

[93] B. Struth, A. Snigirev, O. Konovalov, A. Otten, R. Gauggel, and T. Pfohl. Application of Microfocussing at a Nonspecific Beamline. AIP Conferenence Proceceedings, 705:804-807, 2004.

[94] A. Snigirev, V. Kohn, I. Snigireva, and B. Lengeler. A compound refractive lens for focusing high-energy X-rays. Nature, 384:49-51, 1996.

[95] B. Lengeler, C. Schroer, B. Brenner, A. Gerhardus, T. Gunzler, M. Kuhlmann, J. Meyer, and C. Zimprich. Parabolic refractive X-ray lenses. Journal of Synchrotron Radiation, 9:119-124, 2002.

[96] M. Doi and S.F. Edwards. The Theory of Polymer Dynamics. Oxford University Press, 1986.

[97] M. Doi. Introduction to Polymer Physics. Oxford University Press, 1996.

[98] P.J. Flory. Statistical Mechanics of Chain Molecules. Interscience Publishers, New York, 1969.

[99] C. Bustamante, J. F. Marko, E. D. Siggia, and S. Smith. Entropic Elasticity of $\lambda$-Phage DNA. Science, 265:1599-1600, 1994.

[100] O. Kratky and G. Porod. Röntgenuntersuchung gelöster Fadenmoleküle. Recueil des travaux chimiques des Pays-Bas, 68:1106-1122, 1949.

[101] L. D. Landau and E. M. Lifshitz. Statistical Physics. Pergamon Press, London, 1958.

[102] S. Köster. Semiflexible Polymere unter räumlicher Einschränkung am Beispiel des zytoskeletalen Proteins Aktin. Diploma Thesis, University of Ulm, 2003. 
[103] J. Kierfeld. Strings and Filaments: From Vortices to Biopolymers. Habilitation Thesis, University of Potsdam, 2006.

[104] J. Kierfeld and H. Stark. private communications.

[105] J. Kierfeld and R. Lipowsky. Unbundling and desorption of semiflexible polymers. Europhysics Letters, 62(2):285-291, 2003.

[106] J. Kiefeld and R. Lipowsky. Duality mapping and unbinding transitions of semiflexible and directed polymers. Journal of Physics A: Mathematical and General, 38:L155-L161, 2005.

[107] J. Kierfeld. private communications.

[108] J. Käs, H. Strey, M. Bärmann, and E. Sackmann. Direct measurement of the wave-vector-dependent bending stiffness of freely flickering actin filaments. Europhysics Letters, 21(5):865-870, 1993.

[109] T. Takebayashi, Y. Morita, and F. Oosawa. Electronmicroscopic investigation of the flexibility of F-actin. Biochimica et Biophysica Acta, 492:357-363, 1977.

[110] J. Hendricks, T. Kawakatsu, K. Kawasaki, and W. Zimmermann. Confined semiflexible polymer chains. Physical Review E, 51(3):2658-2661, 1995.

[111] H. Stöcker. Taschenbuch der Physik. Verlag Harri Deutsch, Thun und Frankfurt am Main, 1998.

[112] P.W. Atkins. Physical Chemistry. Oxford University Press, sixth edition, 1998.

[113] Y. Sun, Z. Luo, A. Fertala, and K.N. An. Direct quantification of the flexibility of type I collagen monomer. Biochemical and Biophysical Research Communications, 295:382-386, 2002.

[114] L. Masaro and X.X. Zhu. Physical models of diffusion for polymer solutions, gels and solids. Progress in Polymer Science, 24:731-775, 1999.

[115] R.A. Waggoner, F.D. Blum, and J.M.D. MacElroy. Dependence of the Solvent Diffusion Coefficient on Concentration in Polymer Solutions. Macromolecules, 26:6841-6848, 1993.

[116] J.S. Mackie and P. Meares. The Diffusion of Electrolytes in a Cation-Exchange Resin Membrane. Proceedings of the Royal Society of London A, 232:498-509, 1955. 
[117] W. Schnitzler. Abbildung von Biomolekülen im kontinuierlichen Fluss. Diploma Thesis, University of Ulm, 2004.

[118] L.C. Juncqueira and J. Carneiro. Histology. Springer, Heidelberg, 1996.

[119] M. Wolman and F.H. Kasten. Polarized light microscopy in the study of the molecular structure of collagen and reticulin. Histochemistry, 85:41-49, 1986.

[120] R.T. Tranquillo, T.S. Girton, B.A. Bromberek, T.G. Triebes, and D.L. Mooradian. Magnetically oriented tissue-equivalent tubes: application to a circumferentially oriented media-equivalent. Biomaterials, 17:349-357, 1996.

[121] D.P. Knight, X.W. Hu, L.J. Gathercole, M. Rusaouen-Innocent, M.-W. Ho, and R. Newton. Molecular Orientation in an Extruded Collageneous Composite, the Marginal Rib of the Egg Capsule of the Dogfish Scyliorhinus Canicula; A Novel Lyotropic Liquid Crystalline Arrangement and its Origin in the Spinnerets. Philosophical transactions: Biological Sciences, 351:1205-1222, 1996.

[122] S. Newman, M. Cloitre, C. Allain, G. Forgacs, and D. Beysens. Viscosity and Elasticity During Collagen Assembly In Vitro: Relevance to Matrix-Driven Translocation. Biopolymers, 41:337-347, 1997.

[123] S. Köster, J.B. Leach, J.Y. Wong, and T. Pfohl. Microaligned collagen matrices by hydrodynamic focusing: controlling the $\mathrm{pH}$-induced self-assembly. $M R S$ Proceedings, 898E:0898-L05-21, 2005.

[124] J.M. Cassel, L. Mandelkern, and D.E. Roberts. The Kinetics of the Heat Precipitation of Collagen. Journal of the American Leather Chemists Association, 57(11):556-575, 1962.

[125] A. Miller and D. Tocchetti. Calculated X-ray diffraction pattern from a quasihexagonal model for the molecular arrangement in collagen. International Journal of Biological Macromolecules, 3(1):9-18, 1981.

[126] R.D.B. Fraser and T.P. McRae. Unit cell and molecular connectivity in tendon collagen. International Journal of Biological Macromolecules, 3(3):193-200, 1981. 



\section{Acknowledgements}

All denen, die mich während meiner Promotionszeit unterstützt haben, möchte ich an dieser Stelle ganz herzlich danken.

Allen voran bin ich Thomas Pfohl sehr dankbar für seine erstklassige Betreuung. Er hatte wirklich immer Zeit, Antworten auf Fragen und Lösungen für Probleme zu finden, und hat mir während der vergangenen Jahre sehr viel beigebracht. Von dem Aufenthalt in Boston, den er mir ermöglicht hat, habe ich sehr profitiert. Danke auch für die vielen Möglichkeiten, bei Konferenzen meine Arbeit vorzustellen und Kontakte zu knüpfen. Ebenso bin ich froh über all die motivierenden Ermutigungen während meiner Arbeit, die zahlreichen ausführlichen Diskussionen und die gute Zusammenarbeit. Ich möchte mich besonders bedanken bei Stephan Herminghaus. Er gab mir die Gelegenheit, meine Doktorarbeit in seiner Abteilung anzufertigen. Die tolle Ausstattung des Instituts und seine Bereitwilligkeit, mich wo es ging zu unterstützen, hat vieles erleichtert und manches erst ermöglicht. Tim Salditt hat sich bereit erklärt, das Zweitgutachten für meine Arbeit anzufertigen. Er hat mich mit seinem Interesse an meiner Wissenschaft sehr motiviert. Dankbar bin ich auch, dass Erwin Neher, Carsten Ronning, Hans Hofsäss und Detlev Schild als Prüfer zur Verfügung standen und dass sie bei der Absprache von Prüfungsfächern und -terminen sehr flexibel waren und sich alles so problemlos organisieren ließ. Meinen Kollegen der Arbeitsgruppe in Göttingen, Dagmar Steinhauser, Heather

Evans, Semra Öztürk und Rolf Dootz, möchte ich für die gute Atmosphäre in der Gruppe, ob beim Ausflug im Schnee oder beim Arbeiten im Labor, und den tollen Zusammenhalt danken. Heather, Dagmar und Rolf danke ich außerdem für die kritische Korrektur dieser Arbeit. Semra hat immer dafür gesorgt, dass unser Laborbetrieb problemlos laufen konnte. Udo Krafft hatte für alle technischen Probleme eine gute Lösung parat und hat geduldig geholfen, auch wenn Wissenschaftler manchmal jeden Tag eine neue Idee haben. Vielen Dank dafür. Danke auch an Monika Teuteberg - dafür, dass sie alle organisatorischen Dinge perfekt im Griff hatte und mir immer weiterhelfen konnte. Ich war immer sehr dankbar, dass HansHerbert Krull sich aller Computerprobleme angenommen hat und immer eine 
Lösung fand. Oliver Adolph danke ich für die kritische Korrektur der Arbeit und insbesondere der Abbildungen. Jan Kierfeld hat mir sehr geholfen, die theoretischen Aspekte der Arbeit zu verstehen und hatte immer die Zeit und Geduld, meine Fragen zu beantworten. Auch mit Holger Stark, Erwin Frey, Frederik Wagner und Klaus Mecke habe ich wichtige Diskussionen zur theoretischen Beschreibung meiner Experimente geführt, die mir neue Aspekte aufgezeigt und mich auf dem Weg zur richtigen Erklärung weiter gebracht haben. Ohne Bernd Struth, Oleg Konovalov und Anatoly Snigirev wären die Experimente am ESRF Grenoble nicht möglich gewesen. Sie haben alles ihnen Mögliche dafür getan, dass wir optimale Ergebnisse erzielen konnten. Manuela Pluntke, Lothar Zitzler, Alexander Otten, Thomas Becker, Daniel Podzimek, Jean-Christophe Baret und Jürgen Bührle haben sehr dazu begetragen, dass meine Zeit in Ulm richtig schön und die Stimmung in der Abteilung Angewandte Physik immer gut war. Ich bin froh, dass diese Freundschaften die Umzüge in alle Himmelsrichtungen überdauert haben. Joyce Wong hat mir während sieben toller Monate in Boston alle Freiheiten gelassen, und mir gleichzeitig ermöglicht wirklich viel zu lernen. Dankbar bin ich auch, dass sie mich beim Knüpfen neuer Kontakte sehr unterstützt hat. Wynter Duncanson, Jennie Leach, Xin Brown, Sooyoung Kim und Mike Figa waren nicht nur Kollegen, die mir immer sehr geduldig geholfen haben und mit denen ich gerne zusammengearbeitet habe, sondern sind auch zu guten Freunden geworden. Danke auch an meine Mitbewohner in Boston, Mette Møller-Kristensen, Nadia Georgieva und Simon Lindenmann. Ohne sie wäre meine Zeit dort nur halb so schön gewesen. Schließlich möchte ich meinen Eltern und Schwestern dafür danken, dass ich mich immer auf sie verlassen konnte und kann. 


\section{Curriculum Vitae}

\section{Persönliche Angaben:}

Name:

Geburtstag:

Geburtsort:

Staatsangehörigkeit:
Sarah Friederike Köster

25. März 1979

Reutlingen

deutsch

\section{Ausbildung:}

07/2004 - heute: Promotionsstudium (MPI für Dynamik und Selbstorganisation, Göttingen)

06/2004 - 12/2004: Forschungsaufenthalt (Boston University, USA)

07/2003 - 06/2004: Promotionsstudium (Universität Ulm)

04/2003: Diplom in Physik

05/2002 - 04/2003: Diplomarbeit

10/1998 - 04/2003: Physikstudium (Universität Ulm)

06/1998: Abitur

08/1994 - 11/1994: Besuch der Huron Heights Secondary School, Newmarket, Kanada

08/1989 - 06/1998: Besuch des Graf-Eberhardt-Gymnasiums Bad Urach

08/1985 - 07/1989: Besuch der Grundschule Bad Urach

\section{Berufserfahrung:}

07/2003 - heute: Wissenschaftliche Mitarbeiterin an der Universität Ulm und dem MPI für Dynamik und Selbstorganisation Göttingen, Gastwissenschaftlerin an der Boston University

09/2001 - 10/2001: Forschungsaufenthalt (University of Sussex at Brighton, UK)

05/2000 - heute: Verschiedene Tätigkeiten als wissenschaftliche Hilfskraft an den Universitäten Ulm und Göttingen (Mathematik, Physik)

08/1998 - 09/1998: Praktikum bei der Robert Bosch GmbH, Reutlingen

\section{Stipendien:}

01/1999 - 06/2003: Studienstiftung des deutschen Volkes

07/2001- 06/2003: Siemens AG Student Program (SSP) 